\title{
A WiFi Tracking Device Printed Directly on Textile for Wearable Electronics Applications
}

\author{
Thesis by \\ Bauyrzhan Krykpayev
}

In Partial Fulfillment of the Requirements

For the Degree of

Masters of Science

King Abdullah University of Science and Technology, Thuwal,

Kingdom of Saudi Arabia

December, 2015 
The thesis of Bauyrzhan Krykpayev is approved by the examination committee

Committee Chairperson: Assist. Prof. Dr. Atif Shamim

Committee Member: Assoc. Prof. Dr. Muhammad M. Hussain

Committee Member: Assoc. Prof. Dr. Jr-Hau He 
Copyright (C)2015

Bauyrzhan Krykpayev

All Rights Reserved 


\title{
ABSTRACT
}

\section{A WiFi Tracking Device Printed Directly on Textile for}

\author{
Wearable Electronics Applications
}

\section{Bauyrzhan Krykpayev}

Wearable technology is quickly becoming commonplace in our everyday life - fitness and health monitors, smart watches, and Google Glass, just to name a few. It is very clear that in near future the wearable technology will only grow. One of the biggest wearable fields is the E-textiles. E-textiles empower clothes with new functionality by enhancing fabrics with electronics and interconnects. The main obstacle to the development of E-textile field is the relative difficulty and large tolerance in its manufacturing as compared to the standard circuit production. Current methods such as the application of conductive foils, embroidering of conductive wires and treatment with conductive coatings do not possess efficient, fast and reliable mass production traits inherent to the electronic industry. On the other hand, the method of conductive printing on textile has the potential to unlock the efficiency similar to PCB production, due to its roll-to-roll and reel-to-reel printing capabilities. Furthermore, printing on textiles is a common practice to realize graphics, artwork, etc. and thus adaptability to conductive ink printing will be relatively easier. Even though conductive printing is a fully additive process, the end circuit layout is very similar to the one produced via PCB manufacture. However, due to high surface roughness and porosity of textiles, efficient and reliable printing on textile has remained elu- 
sive. Direct conductive printing on textile is possible but only on specialized dense and tightly interwoven fabrics. Such fabrics are usually uncommon and expensive. Another option is to employ an interface layer that flattens the textile surface, thus allowing printing on it. The interface layer method can be used with a variety of textiles such as polyester/cotton that can be found in any store, making this method promising for wearable electronics. Very few examples and that too of simple structures such as a line, square patch or electrode have been reported which utilize an interface layer [1-13]. No sophisticated circuit or a system level design involving integration of components on textile has been demonstrated in this medium before. This work, for the first time, demonstrates a complete system printed on a polyester/cotton T-shirt, that helps in tracking the person who is wearing that T-shirt through a smart phone or any Internet enabled device. A low cost dielectric material (Creative Materials 116-20 Dielectric ink) is used to print the interface layer through manual screen printing method. The circuit layout and antenna have been ink-jet printed with silver nano-particles based conductive ink. Utilizing WiFi technology, this wearable tracking system can locate the position of lost children, senior citizens, patients or people in uniforms, lab coats, hospital gowns, etc. The device is small enough (55 mm x $45 \mathrm{~mm}$ ) and light weight (10.5g w/o battery) for people to comfortably wear it and can be easily concealed in case discretion is required. Field tests have revealed that a person can be localized with up to 8 meters accuracy and the device can wirelessly communicate with a hand-held receiver placed 55 meters away. Future development of the method with techniques such as automated screen printing, pick and place components, and digital ink-jet printing can pave the way for mass production. 


\section{ACKNOWLEDGEMENTS}

I would like to thank my supervisor Professor Atif Shamim for guiding me through the work. His profound expertise, critical judgment, energizing optimism and universal support were instrumental to the success of this thesis. I would like to thank my co-authors Bilal and Fahad for sharing with me their embedded systems and antenna design knowledge respectively.

My sincere appreciation goes to my colleagues at the IMPACT Lab for always willing to help me in myriad ways possible. It is hard to count how many times I have been getting great guidance, advice and thoughts on absolutely different topics ranging from thesis work to life matters. Thank you, guys.

Finally, I would like to thank my parents, Yerlan and Aklima, for always trusting my choices, believing and supporting me. I would like to thank my wife, Raushan, for her endless love and care despite all the time I spend away from her working in the lab. 


\section{TABLE OF CONTENTS}

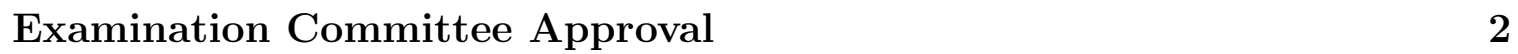

\begin{tabular}{ll}
\hline Copyright & 3 \\
\hline
\end{tabular}

\begin{tabular}{ll}
\hline Abstract & 4
\end{tabular}

\begin{tabular}{|l|l}
\hline Acknowledgements & 6
\end{tabular}

\begin{tabular}{ll}
\hline List of Figures & 10
\end{tabular}

\begin{tabular}{ll}
\hline List of Tables & 13
\end{tabular}

$\begin{array}{lll}1 & \text { Introduction } & 14\end{array}$

1.1 Motivation . . . . . . . . . . . . . . . . . . . . . . . . . . 14

1.2 Objectives . . . . . . . . . . . . . . . . . . . . . . . . 17

1.3 Challenges . . . . . . . . . . . . . . . . . . . 17

1.4 Contributions . . . . . . . . . . . . . . . . . . . . . . . . . . . 18

1.5 Thesis Organization . . . . . . . . . . . . . . . . . . . . 19

2 Smart Fabrics and Localization Overview 21

2.1 Smart Fabrics . . . . . . . . . . . . . . . . . . . . . . . . . . . . . . . 21

2.1 .1 Direct Attachment and Application of Conductive Foil . . . . 23

2.1 .2 Sewing, Embroidering, Knitting, Weaving, etc. . . . . . . . . . 24

2.1 .3 Conductive Printing . . . . . . . . . . . . . . . . 25

2.2 Interface Layer Method . . . . . . . . . . . . . . . . . . . . . . . . . . 28

2.2 .1 Method Overview . . . . . . . . . . . . . . . . . 28

2.2 .2 Existing Applications of Interface Layer Method . . . . . . . 30

2.3 Tracking, Localization or Locating. . . . . . . . . . . . . . . . . . . . 32

2.3 .1 Tracking in wearables . . . . . . . . . . . . . . . . . . . . 32

2.3 .2 Tracking techniques . . . . . . . . . . . . . . . . . . . . . . 34

2.4 Conclusion . . . . . . . . . . . . . . . . . . . . . . . . . . . . 35 
3 The Interface Layer Method: Requirements, Fabrication and Char$\begin{array}{ll}\text { acterization } & 38\end{array}$

$3.1 \quad$ Interface Method Requirements . . . . . . . . . . . . . . . . . . . . . 39

$3.1 .1 \quad$ Interface Layer Material Selection . . . . . . . . . . . . . . . . 39

3.1 .2 Fabric Selection . . . . . . . . . . . . . . . . . . . . 41

3.1 .3 Interface Layer Application Method . . . . . . . . . . . . . . . 42

3.1.4 Conductive Printing . . . . . . . . . . . . . . . . 46

3.2 Interface Layer Fabrication . . . . . . . . . . . . . . . . . . 47

3.2 .1 Fabink-UV-IF4 . . . . . . . . . . . . . . . . . . . . . 47

3.2.2 CM116-20 dielectric paste . . . . . . . . . . . . . . . . 51

3.2.3 Fabink-UV-IF4 and CM116-20 Comparison . . . . . . . . . . 53

3.3 Characterization . . . . . . . . . . . . . . . . . 57

3.3.1 Electrical Properties of Conductively Printed Layers on Interface Layer . . . . . . . . . . . . . . . . . . . . . . . . . . 59

3.3 .2 Conductive Trace Resolution . . . . . . . . . . . . . . . . 63

3.3 .3 RF Properties of Interface Layer. . . . . . . . . . . . . . . . . 65

3.3 .4 Bending analysis . . . . . . . . . . . . . 66

3.4 Summary . . . . . . . . . . . . . . . . . . . 67

4 Localization Device: Design, Fabrication, and Testing 69

4.1 Prototyping . . . . . . . . . . . . . . . . . . 69

4.1 .1 System Design . . . . . . . . . . . . . . . . . 70

4.1 .2 Prototype Hardware . . . . . . . . . . . . . . . . . 72

4.1 .3 Prototype Firmware and Software . . . . . . . . . . . . . . . . 74

4.2 On-Textile Fabrication . . . . . . . . . . . . . . . . . . . . . . . . . . . 76

$4.2 .1 \quad$ On-Textile Antenna . . . . . . . . . . . . . . . . . . . . . . . . 77

4.2 .2 On-Textile Prototype . . . . . . . . . . . . . . . . . . . . . 84

4.3 Field Tests . . . . . . . . . . . . . . . . . . . . . . . . . . . . . . . . . . . . 87

4.3 .1 Device Radiation Pattern . . . . . . . . . . . . . 87

4.3 .2 Communication Distance . . . . . . . . . . . . . . . . . . . . . 91

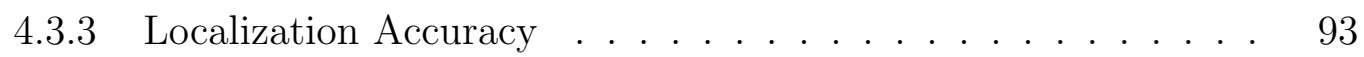

4.4 Final Device . . . . . . . . . . . . . . . . . . . . . . . . . . . . . . . . . . . . . . 99

4.5 Summary . . . . . . . . . . . . . . . . . . . . . . . . . 101

$\begin{array}{lll}5 & \text { Concluding Remarks } & 104\end{array}$

5.1 Summary . . . . . . . . . . . . . . . . . . . 104

5.2 Future Research Work . . . . . . . . . . . . . . . . . . . . . . . . 105 
\begin{tabular}{ll}
\hline References & 107
\end{tabular}

\begin{tabular}{|ll}
\hline Appendices & 115
\end{tabular} 


\section{LIST OF FIGURES}

1.1 Projected growth of connected wearable devices |14] . . . . . . . . . 14

1.2 Estimated smart cloting and body sensors revenue 2013-2020 [15] . . 15

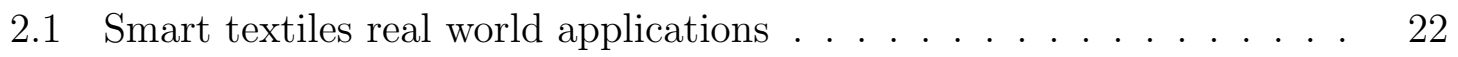

2.2 Direct Attachment and Conductive foil . . . . . . . . . . . . . . 23

2.3 Conductive thread implementations $[16] \ldots \ldots \ldots \ldots . \ldots . \ldots 24$

2.4 Embroidded electronics . . . . . . . . . . . . . . . . . . . . . . . 25

2.5 Common conductive printing techniques $\ldots \ldots \ldots \ldots \ldots$

$2.6 \quad$ SEM image of $65 / 35$ polyester/cotton $\ldots \ldots \ldots \ldots \ldots . \ldots \ldots 27$

2.7 Direct printing on textiles $\ldots \ldots \ldots \ldots \ldots$

$2.8 \quad$ Conductive printing on textile using interface layer [1] $\ldots \ldots \ldots . \quad \ldots 29$

$2.9 \quad$ Existing applications of interface layer method $[1[12] \ldots \ldots \ldots$

2.10 Multitude of wearable tracking devices [17] . . . . . . . . . . 32

2.11 Tracking gadgets on ordinary clothes. . . . . . . . . . . 33

2.12 GPS, GSM and WiFi . . . . . . . . . . . . . . . . . 35

$3.1 \quad$ Dielectric inks - Fabink-UV-IF4 on left side, Creative Materials 116-20

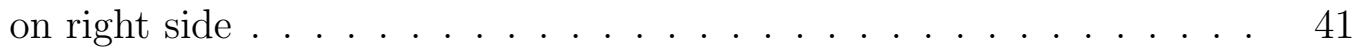

$3.2 \quad$ Textile substrates $100 \%$ cotton (left), $65 \% / 35 \%$ polyester/cotton (middle) and $85 \% / 15 \%$ polyester/cotton(right). . . . . . . . . . 41

$3.3 \quad$ Common screen printing process $[18] \ldots \ldots \ldots \ldots \ldots \ldots \ldots$

3.4 Screen printing accessories: a) 3 layer PET stencil b) D-cut squegee . 45

3.5 Manual screen printing process: a)top view b)side view . . . . . . . 45

3.6 Ink-jet printing procedure $\ldots \ldots \ldots \ldots \ldots$

$3.7 \quad$ Interface layer formation steps using Fabink-UV-IF4 a) Set up b) dielectric paste application c) first squeegee pass d) second squegee pass e) UV curing . . . . . . . . . . . . . . . . . . . . . . . . . 49

$3.8 \quad$ The interface layer formed using Fabink-UV-IF4. The dotted line was added for easier visualization. . . . . . . . . . . . . . . 50

3.9 Conductive lines printed on the Fabink-UV-IF4 interface layer. . . . 50 
3.10 Diffussion of conductive ink into CM116-20 interface layer. The dotted line was added for easier visualization. . . . . . . . . . . . . . . . . . 51

3.11 The interface layer formed using CM116-20. . . . . . . . . . . . . 53

3.12 Interface layer bending: a)Fabink-UV-IF4 b)CM116-20 . . . . . . . . 54

3.13 SEM of interface layers: a)Fabink-UV-IF4 b)CM116-20 . . . . . . . . 54

3.14 AFM of interface layers: a)Fabink-UV-IF4 b)CM116-20 . . . . . . . . 55

3.15 Deposited conductive layout of the localization device. . . . . . . . . 57

3.16 CM116-20 interface layer on: a) $65 \% / 35 \%$ polyester/cotton b) $100 \%$ cot-

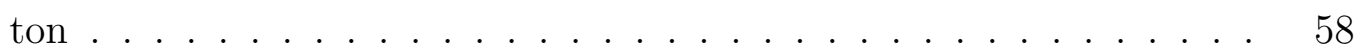

3.17 AFM of interface layer on: a) $65 \% / 35 \%$ polyester/cotton b) $100 \%$ cotton 58

3.18 Demonstrating flexibility of: a) $65 \% / 35 \%$ polyester/cotton b) $100 \%$ cotton . . . . . . . . . . . . . . . . 59

3.198 lines and 8 rectangles for electric properties measurements. . . . . . 61

3.20 Conductivity dependency on the number of layers. . . . . . . . . . . . 62

3.21 Minimum trace width measurement. . . . . . . . . . . . . . . . 64

3.22 Measurement of: a) permittivity $\left(\varepsilon_{r}\right)$ b) loss tangent $(\tan \delta)$. . . . . . 65

3.23 Interface layer bending to check structure conductivity a) front view b) side view . . . . . . . . . . . . . . . . 66

4.1 System design of the localization device . . . . . . . . . . . . . . 70

4.2 Localization prototype schematic . . . . . . . . . . . . . . . 73

4.3 Localization prototype: a) circuit layout b) final PCB prototype . . . 74

4.4 Standard inverted F-antenna simulated in HFSS . . . . . . . . . . . . 78

4.5 Localization circuit's antenna design and dimensions in HFSS . . . . 79

$4.6 \quad$ Fabricated IFA a) Front view with connector cable b) Antenna placed in anechoic chamber . . . . . . . . . . . . . . 80

4.7 Reflection coefficients, S11, of fabricated antenna and HFSS simulation 81

4.8 Radiation pattern of a) HFSS simulation b) fabricated antenna. . . . 82

4.9 Radiation pattern cross-section of a) E-plane b) H-plane . . . . . . . 82

4.10 Additional current path contributed by extra beam . . . . . . . . . . 83

4.11 The final localization circuit design a) schematic b) layout . . . . . . 84

4.12 Tracking device fabrication process a) printing circuit layout b) components attachment . . . . . . . . . . . . . 86

4.13 On-textile prototype for field tests powered from LiPo battery . . . . 87

4.14 Device radiation pattern measurements . . . . . . . . . . . . 88

4.15 Radiation pattern measurements in free space a) E-plane b) H-plane. 89 
4.16 Radiation pattern measurements on body a) E-plane b) H-plane . . . 89

4.17 Measured E-plane and H-plane on a) free space b) body. . . . . . . . 89

4.18 Communication distance measurements on-body at a) 0 meters b) 40

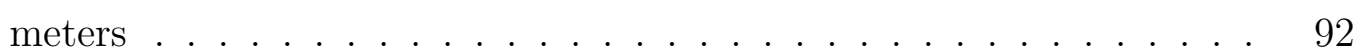

4.19 Far field communication distance measurement results . . . . . . . . . 93

4.20 Plotting the position of the device on the map . . . . . . . . . . . . . 94

4.21 Localization accuracy testing process . . . . . . . . . . . . 96

4.22 The deviation from the original position for on-body and in air measurements . . . . . . . . . . . . . . . . . . . . 97

4.23 Distance deviation between the actual position and the measured position for in air, on body and 25 access points with provided Google accuracy values(grey bars) . . . . . . . . . . . . . . . . . . . . . 98

4.24 The final localization device attached on T-shirt . . . . . . . . . . . . 100

4.25 The overall flexibility of the final localization device . . . . . . . . . . 101 


\section{LIST OF TABLES}

$2.1 \quad$ Characteristics Comparison of E-textiles manufacturing methods . . . 36

3.1 Fabink-UV-IF4 and CM 116-20 Data . . . . . . . . . . . . . . . . . . 40

3.2 Properties of textile substrates . . . . . . . . . . . . . . . . . 42

3.3 Fabink-UV-IF4 and CM116-20 Comparison . . . . . . . . . . . . 56

3.4 Electrical properties of conductive layers on $85 \% / 15 \%$ polyester/cotton. 61

$3.5 \quad$ Electrical properties of conductive layers on $65 \% / 35 \%$ polyester/cotton and $100 \%$ cotton substrates . . . . . . . . . . . . . 63

$3.6 \quad$ DC resistance of traces with different width . . . . . . . . . . . . . . 64

3.7 Permittivity and loss tangent for three textile substrates . . . . . . 66

3.8 Bending Impact on DC resistance . . . . . . . . . . . . . . . . 67

$4.1 \quad$ Inverted F-antenna parameters simulated in HFSS . . . . . . . . . . . 79

4.2 Fabricated on-textile IFA parameters . . . . . . . . . . . . . . . 83

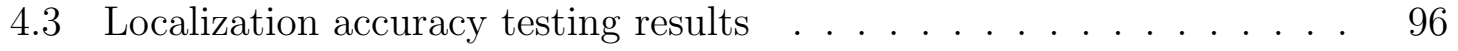

4.4 Bill of materials of the localization device . . . . . . . . . . . . . . . 100

4.5 The parameters of the localization device build on textile substrate via the Interface Layer method . . . . . . . . . . . . . . . . . . . . . . . . 102 


\section{Chapter 1}

\section{Introduction}

\subsection{Motivation}

Wearable devices are taking the world by storm. Cisco estimates 14 that by 2019 number of wearable devices in the world that can connect to Internet will increase almost sixfold from 109M in 2014 to 578M devices in 2019 (Fig,1.1). E-textiles, also known as smart fabrics, smart textiles or smart clothing, make up a considerable part of the wearable technology field. Global Information Research suggests [15] that E-textiles market will produce revenue of USD 600 million by the year 2020 (Fig.1.2). Such growth is very impressive, but it only can be supported by the efficient mass production of smart textiles.

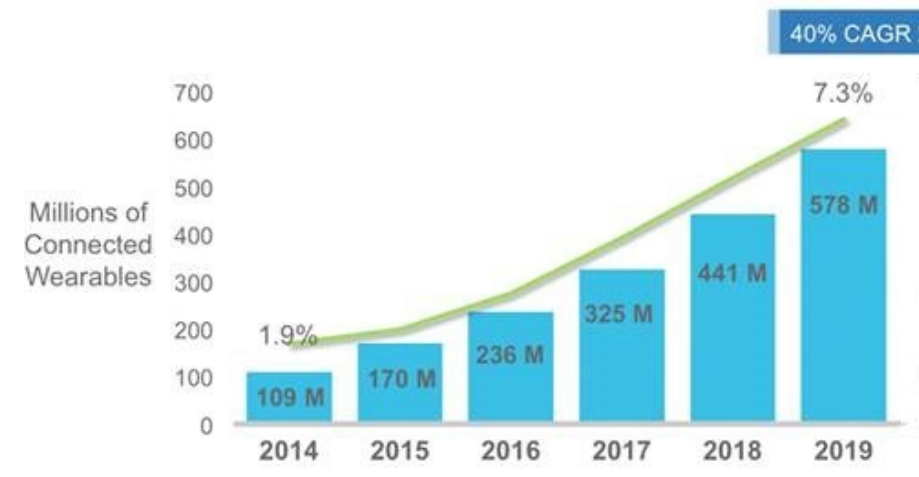

Figure 1.1: Projected growth of connected wearable devices 14 
15

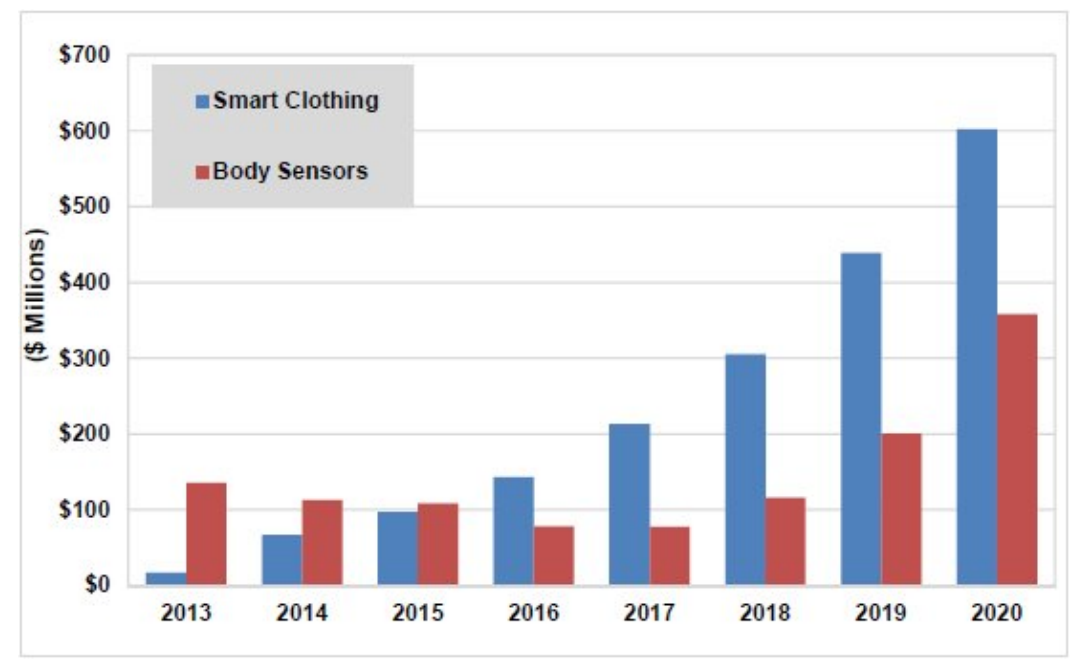

Figure 1.2: Estimated smart cloting and body sensors revenue 2013-2020 [15

Methods of smart textile manufacturing vary but they roughly can be divided into three categories:

- application of conductive foils (copper, aluminum, etc.) onto textile 1921

- knitting, weaving or embroidering fully conductive threads or conductive coatings into the fabric 2225

- conductive printing on textiles $1,13,2637$

All three categories are perfectly viable production methods and have their uses, advantages and shortcomings. However, only conductive printing on textile can potentially acquire efficient, fast and reliable mass production traits inherent to the electronic industry. Similar to PCB manufacturing, conductive printing produces a circuit layout on top of textile substrates. Discrete components can be bonded to the layout to complete the device production cycle. However, due to high surface roughness and porosity of most of the textiles, the direct conductive printing is feasible only on very dense and tightly interwoven fabrics [26 37]. 
Another option is to print an interface layer that flattens the textile surface preparing it for subsequent conductive printing. The interface layer method, initially proposed by researchers at the University of Southampton [1 13$]$, can be used with a variety of textiles such as polyester/cotton. Such fabric is cheap and easy to acquire, making this method very accessible and promising for wearable electronics. Up until now the conductive printing via interface layer method was limited to simple structures such as lines, patches, electrodes, and simple antennas $[1-13]$. No complete circuit design involving an integration of electronic components on textile has been demonstrated in this medium before. Furthermore, the primary ingredient in interface layer method is the interface layer ink. Current ink Fabink UV IF-4 which is used in every interface layer method research paper [1-13] is much more expensive than regular dielectric inks. The high cost of the current interface layer ink is prohibitive, and research attempts should be made to optimize a regular dielectric ink to function with textiles.

Localization (tracking, locating) is a well-studied concept that has many applications in different fields such as GPS, phone applications, avionics, etc. There are several examples of clothes that feature tracking devices [38]. However, in these applications trackers are standalone devices that are attached, glued or pocketed on or within clothes. While tracking performance of such device is generally satisfying, the physical feel of an extrinsic PCB-based gadget is rough, cumbersome and abnormal to textiles. Most of these devices are incorporated into heavy duty clothes such as jackets, coats or uniforms, and are not suitable for regular clothing such as T-shirts or dresses. Removing PCB substrate and attaching discrete components directly to a printed circuit layout on fabric using an interface layer method has never been done before and will render localization devices much less obtrusive and conspicuous. Interface layer printing method can potentially allow mass production of localization devices printed on T-shirts, uniforms, lab coats, hospital gowns, etc. 
This thesis investigates the interface layer method to incorporate a localization device printed directly onto fabric. Using WiFi protocol such localization device can help tracking lost children, senior citizens, patients, workers, etc. via any Internetenabled device.

\subsection{Objectives}

The goals of this work are listed below:

- Build a fully functional localization device on textile including its hardware, firmware and software.

- Develop a reliable process of ink-jet printing complete circuit layout on textile using the Interface Layer method.

- Optimize a regular low-cost dielectric ink to work on clothes via the Interface Layer method.

- Perform comprehensive characterization of localization device and antenna both in free space and on-body.

\subsection{Challenges}

Throughout the course of this work the following challenges are expected to be encountered:

- All previous research devoted to the Interface Layer method focused only on the formation of an interface layer via the specially designed interface layer ink. However, the employment of a general purpose dielectric paste has never been researched before. It is expected that the interface layer fabrication process 
utilizing a general purpose dielectric paste will be procedurally different, thus challenging.

- Since conductive printing has never been accomplished on an interface layer made from a general purpose dielectric ink, necessary conductive printing characterization should be first tackled before circuit layouts can be reliably printed.

- The bonding of small-sized electronic components such as SMD chips to a circuit layout printed on an interface layer can be challenging due to the high elasticity of an interface layer and textile that requires bonding to be strong enough to withstand constant bending.

- Since it is E-textiles, the final tracking device will be worn on a human body. Therefore, the device needs to characterized and tested for the successful operation while being worn. Since a human body emits biomagnetic waves, it will be challenging to quantify the influence of a human body onto device's operation.

\subsection{Contributions}

The thesis work contributes to the development of E-textiles field with the following findings:

- The fully functional localization device incorporated directly onto textile using interface layer method for wearable electronics applications.

- The complete circuit layout ink-jet printed on a textile substrate via interface layer method incorporating interconnects, antenna and a ground plane.

- The optimized process of interface layer formation using relatively cheap general purpose dielectric ink Creative Materials 116-20 and manual screen printing method. 
- Comprehensive field tests of the localization device and inverted f-type antenna in free space and on-body at $2.4 \mathrm{GHz}$ presenting the radiation patterns, RSSI, transmitting distance and localization accuracy.

\subsection{Thesis Organization}

The thesis is organized according to the following description:

- Chapter 2 provides an overview of E-textiles and its manufacturing technologies. Advantages and disadvantages of each technology are summarized. Next, the printing on textile methods such as direct printing and interface layer printing are explored in greater details including their applications, shortcomings, and previously published work. Finally, the brief overview of existing localization methods and their applications is presented.

- Chapter 3 studies the peculiarities of printing on fabric via interface layer method. Various aspects such as textile and dielectric ink optimization, application, and processing of interface layer are examined in greater detail. The novel approach of utilizing regular dielectric ink for interface layer formation is described. Finally, electric characteristics of the interface layer and conductive lines are measured and presented.

- Chapter 4 discusses the fabrication and characterization of a fully working localization device. Rapid prototyping using PCB, sample firmware, and server side code are shortly presented in this chapter. After that, inverted F-antenna simulation, fabrication, and RF characterization are demonstrated. Next, the printing of complete circuit layout, bonding of discrete elements and troubleshooting the circuit on textile is summarized. Finally, free space and on-body test field procedures and results are announced. 
- Chapter 5 summarizes the main thesis findings, discusses the possible future applications and suggests ideas for future research. 


\section{Chapter 2}

\section{Smart Fabrics and Localization}

\section{Overview}

\subsection{Smart Fabrics}

Smart textiles is a term used for wearable technology on a textile platform that incorporates electronics, interconnects, sensors, etc. on or within fabrics allowing for facilitated human-device interaction. Smart textiles market is on the rise - Grand View Research estimates that the global smart textile market will exceed USD 1,500 million by 2020 [39]. Application fields are very broad, most notably:

- Personal Protective Equipment such as "WASP" firefighters' sensory system [40], "Safe@Sea" personal protective suit for fishing industries [41].

- Military and Defense such as "Sagem Felin" modernized combat suit with an integrated rechargeable battery and wireless technology [42].

- Space applications such as "Smart Sock" [43] shown on Fig 2.1(b) that monitors the efficiency of training exercises in space or Astroskin's "Smart T-shirt" 44 that analyses astronaut's health parameters.

- Sports and health applications [45], [3] such as heart rate monitors and multi- 
parameter sensor monitors (Fig,2.1(a) developed by number of companies.

- Fashion and Light Emitting Textiles such as wearable solar dress designed by Pauline van Dongen [46] and Switch Embassy's TshirtOS (Fig 2.1(c) that can output short messages directly on the T-shirt 47].

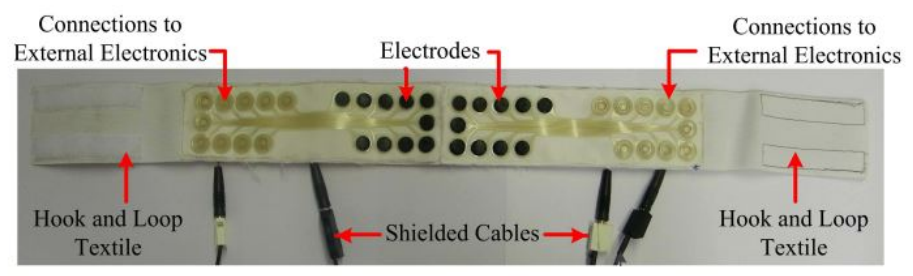

(a) ECG Monitor 3

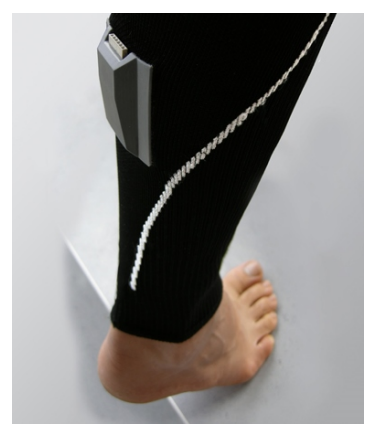

(b) Smart Socks 43

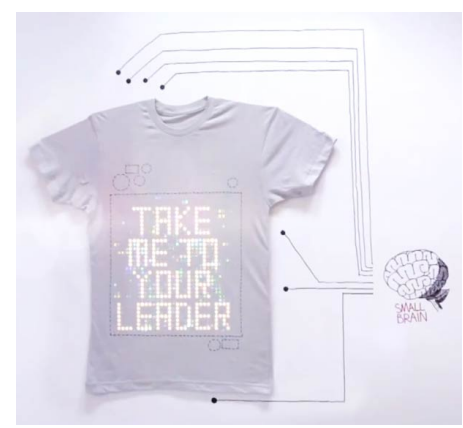

(c) Smart T-shirt 47]

Figure 2.1: Smart textiles real world applications

Smart textile application is diverse, and so are their production methods. In general, E-textiles manufacturing can be divided into three broad categories:

- Direct attachment and application of conductive foil.

- Embroidering, knitting, weaving, Sewing, etc.

- Conductive printing

All these methods have their advantages and shortcomings that will be discussed in the next sections. 


\subsubsection{Direct Attachment and Application of Conductive Foil}

Direct attachment of electronics to clothes is a very simple manufacturing method. Pre-made electronic devices are stitched, glued, or put into the compartments on clothes similar to Fig 2.2(a), Such method is easy to replicate, but it essentially does not attempt any integration of electronics and fabrics. Thus, the direct attachment method usually ignores the textile nature since traditional electronic circuits are generally rigid and rough while textile is flexible and soft. Even though electronic circuits can be made small in size, it is still a complicated process to fit them on everyday clothes such as T-shirts or dresses. Therefore, the direct attachment method is mostly used to incorporate electronics onto heavy duty clothes where size and flexibility are not decisive factors, such as uniforms, ski jackets and protective apparel[ [48]].

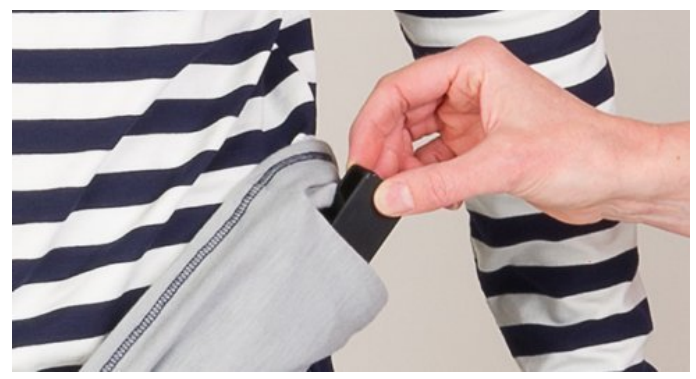

(a) Direct Attachment 49

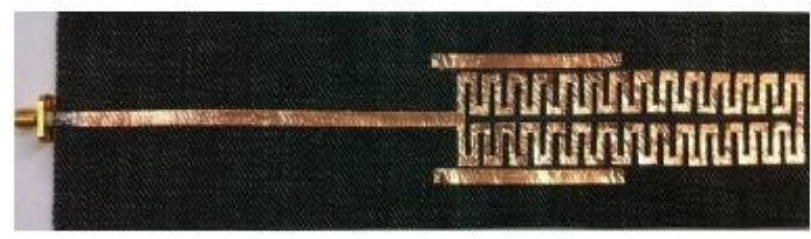

(b) Conductive Foil 20]

Figure 2.2: Direct Attachment and Conductive foil

The application of conductive foil is the next step from the direct attachment method. In this method, a metal foil, that is usually made of aluminum or copper is used to create plain structures such as interconnects, patches or simple antennas by gluing or stitching the foil to a fabric. This method is fast, simple and handy 
for prototyping, DIY(Fig 2.2(b) and even research work 19 21]. Conductive foils are usually flexible, thus can bend along with textile. However prolonged bending, which is usual for clothes, can crack or even rip foils apart from textile. Furthermore, to the author's best knowledge, currently, there are no mass production methods of conductive foils application onto fabric.

\subsubsection{Sewing, Embroidering, Knitting, Weaving, etc.}

Embroidering, sewing, knitting, weaving, etc. are standard clothing manufacturing techniques that have been around for centuries. However, to accommodate for Smart Fabrics production, common textile thread should be enhanced with conductive capabilities via [16:

- Metal wire - a piece of wire that is wrapped along a yarn(Fig.2.3(a).

- Metal fibers - regular thread consisting of conductive filaments(Fig.2.3(b) .

- Metal coating - yarn is chemically coated with a thin conductive layer(Fig.2.3(c)].

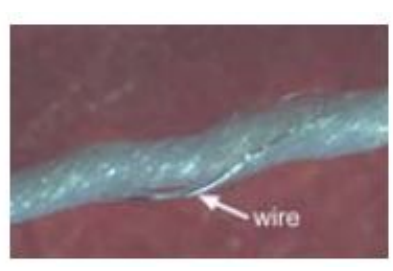

(a) Metal Wire

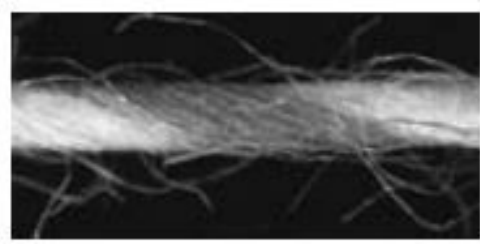

(b) Metal Fibers

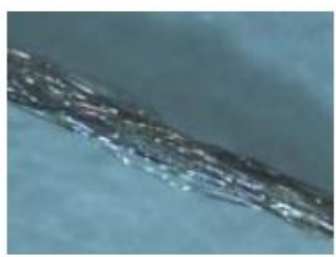

(c) Metal Coating

Figure 2.3: Conductive thread implementations 16

Conductive thread allows to create different electric layouts of varying complexity ranging from simple interconnects and antennas (Fig 2.4(a) to custom fabric structures such as keypads (Fig 2.4(b) . Undeniably, utilization of conductive thread has numerous advantages such as flexibility, innate ability to create several layers, relative resistance to washing, relative durability, etc. In general, production of E-textiles using traditional fabrics manufacturing processes such as embroidery, weaving, sewing, 
etc. is very similar in characteristics to ordinary textile and clothing fabrication. However, while being naturally suitable for textile manufacturing, these methods are very different from common PCB mass production. Interweaving, knitting and embroidering of electric interconnects is seldom uniform and easy and can become very complicated even for simple circuits. Metal threads can potentially rip up, misalign, short or fluff, thus adding to the uncertainty that is detrimental and, in many cases, simply unacceptable for electronic circuits manufacturing. Furthermore, the introduction of conductive wires into textile increments stiffness and lowers elasticity [50].

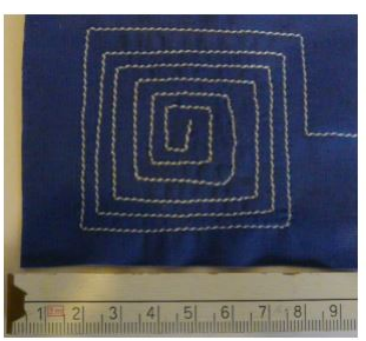

(a) Antenna 51
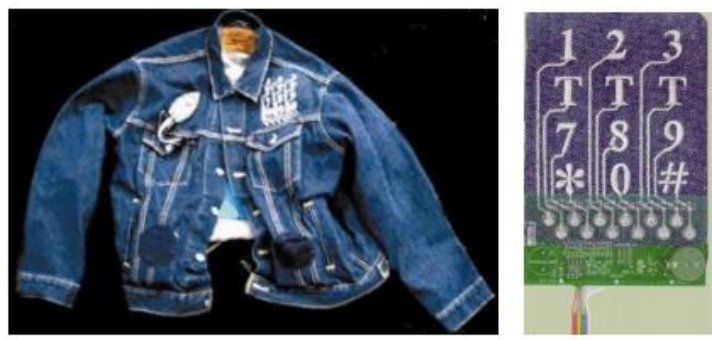

(b) Embroidded keypad on jacket 52

Figure 2.4: Embroidded electronics

\subsubsection{Conductive Printing}

Smart textiles can be produced by the method of conductive printing. This method is already widely exploited to print on different substrates including paper and plastic [53, 54]. The advantage of conductive printing process lies in its similarity to PCB fabrication. Conductive printing allows producing entire electronic circuits in a planar, highly repeatable and low-cost manner. To perform printing, conductive ink that contains metal precursors such as silver or copper is used. Conventional conductive printing technologies are ink-jet and screen printing. Both methods are suitable for mass production with adequate precision. However, ink-jet printing, while being much more flexible and controllable, is usually a slower production method and, thus, is often for prototype manufacturing (Fig,2.5(a). Conductive ink-jet printing 
is very similar to ordinary ink-jet printing process used with common printers, the only difference lies in the printer configuration and the ink type. On the other hand, screen printing is a proven technique for mass production due to its roll-to-roll and reel-to-reel printing capabilities. Moreover, screen printing already can boast a long and successful history of printing graphics, artworks, logos, etc. on different textiles (Fig 2.5(b) . In electronic industry, screen printing is being extensively used to form flexible circuitry on plastic boards [55]. During screen printing, a stenciling process is employed, where a conductive paste is being forced through a patterned screen onto a substrate, thus sketching desired designs. In both ink-jet and screen printing processes, the conductive ink should be sintered or UV-cured after the application.

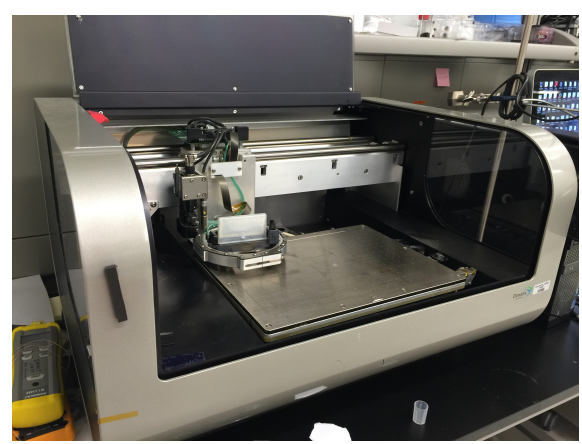

(a) Ink-jet printing

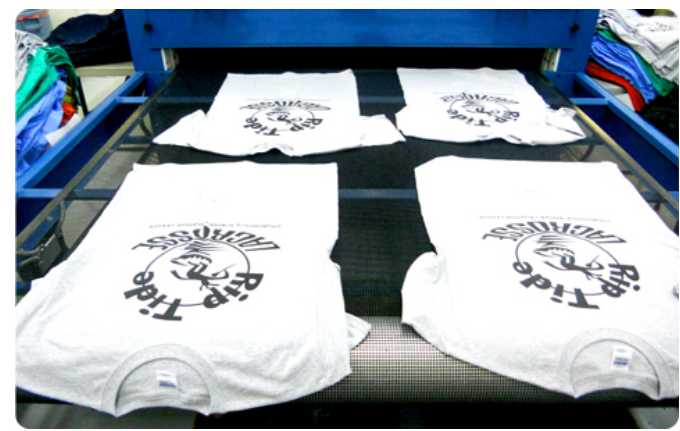

(b) Screen printing on textiles 56

Figure 2.5: Common conductive printing techniques

Conductive printing is a relatively simple process where a conductive ink is deposited onto a substrate in a circuit layout pattern. However, the main obstacle in conductive printing on textiles is the substrate itself. Common textiles are constructed from yarn that is twisted and interwoven. Due to the interlacing of warp and weft yarns, a textile structure is usually very rough and has significant dips that can be clearly seen, such as on Fig.2.6. Such surface structure is highly unfavorable for direct conductive printing on textile since ink will not be distributed evenly across the surface; thus, proper linking will not occur and reasonable conductivity will not be achieved. 


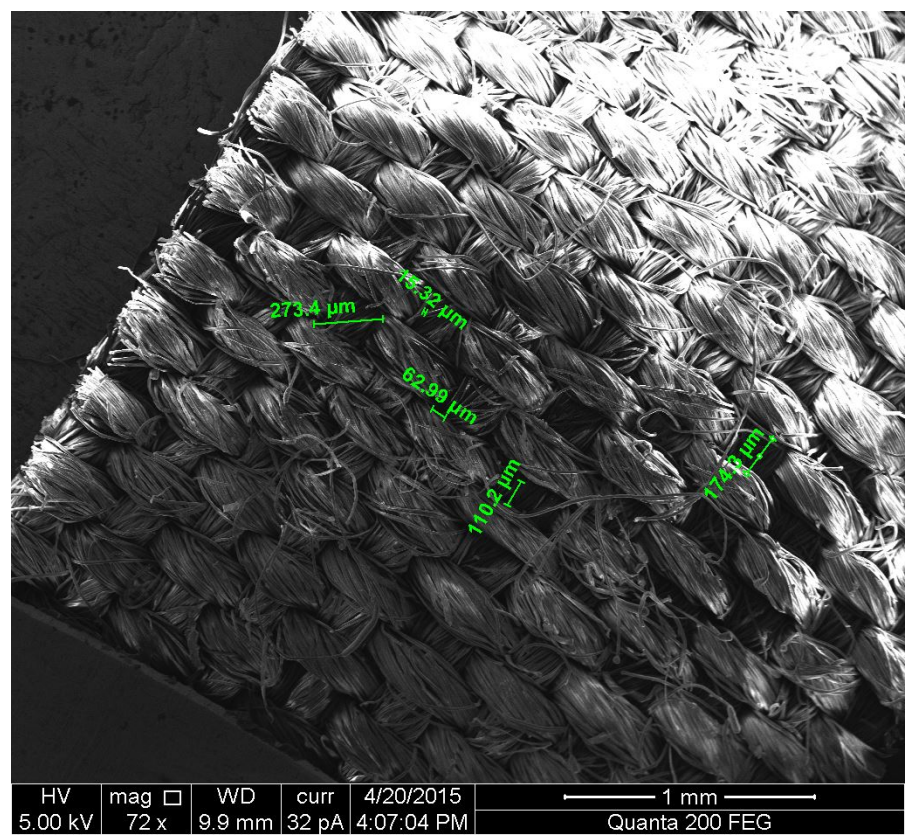

Figure 2.6: SEM image of 65/35 polyester/cotton

Nevertheless, direct printing on woven fabric is possible if textile fibers are thin and densely interwoven. Direct printing on textile was achieved by the research team from Korea Advanced Institute of Science and Technology (KAIST), South Korea 26 34]. In their research work, very fine - tex 4.4 and highly dense - 500 threads/0.1m polyester fabric was used. Such fabric can boast a smooth surface structure that considerably alleviates the conductive printing process. Relatively low interconnect resistivity was achieved that allowed to print various structures such as chip pads, inductors and capacitors (Fig 2.7(a) . Furthermore, researchers from KAIST printed complete circuit layouts and incorporated electronic components such as sensors, LEDs and microcontrollers directly onto printed circuit layouts (Fig 2.7(b)). Such fabric circuit board was termed as the Planar-Fashionable Circuit Board [26]. 


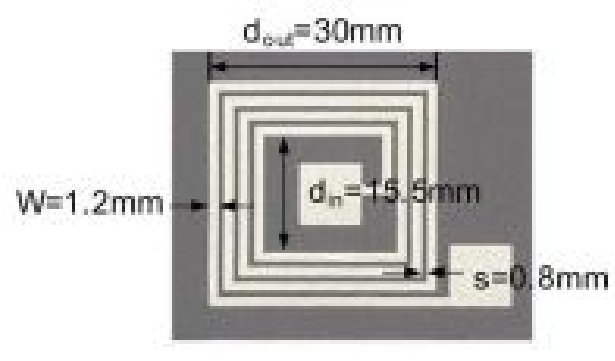

(a) Printed inductor 27

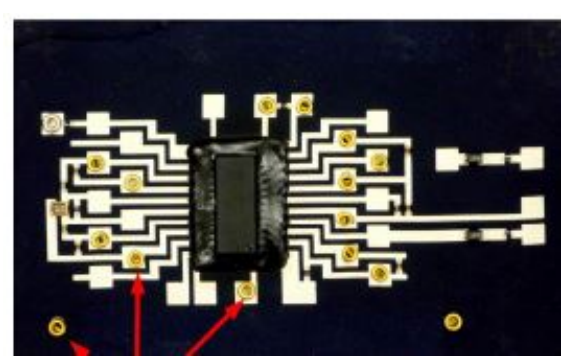

(b) Attached microcontroller 32

Figure 2.7: Direct printing on textiles

Printing directly on textile is a very promising field since it can output circuit boards with similar characteristics to boards produced by classic PCB manufacturing. Furthermore, direct printing on textiles preserves softness and elasticity that is natural to clothes making E-textile clothes generated by this method feel more similar to ordinary everyday clothes. However, the main disadvantage of the direct printing method is that it is limited only to specific fabric - very fine and dense. Such material is uncommon and usually expensive. Even though direct printing method has a lot of potential it cannot be used with regular $100 \%$ cotton or polyester/cotton textiles that are found in regular stores. Thus, direct conductive printing is more of a niche method and not compatible with the majority of the textile types.

\subsection{Interface Layer Method}

Printing on textile is challenging because fabric surface is generally rough and porous. Consequently, to perform successful conductive printing, fabric surface should be made flat and uniform. Interface Layer method focuses on this approach.

\subsubsection{Method Overview}

Originally developed by the researchers from University of Southampton [1 9], UK, interface layer method employs an intermediate screen-printed layer that covers the 
surface profile of a material rendering it flat and relatively smooth [1]. The specially designed polyurethane-based dielectric ink was used to form an interface layer. The detailed process of interface layer formation is depicted on Fig.2.8. The textile material such as polyester/cotton shown on Fig,2.8(a)) is screen printed with an interface dielectric ink (Fig 2.8(b)-(c)) to produce the flat interface layer that smooths the textile surface as described on (Fig $2.8(\mathrm{~d}))$. After that, the interface layer is UV cured(Fig 2.8(e)). Conductive printing is performed by ink-jet printing several layers of conductive ink, usually silver nanoparticle ink(Fig 2.8(f)-(g)). Finally, the conductive ink is thermally cured to produce the final circuit layout(Fig, 2.8 (h)). In this example, ink-jet printing is used for prototype creation.

(a)

(b)

(c)

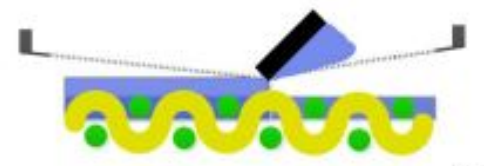

(d)

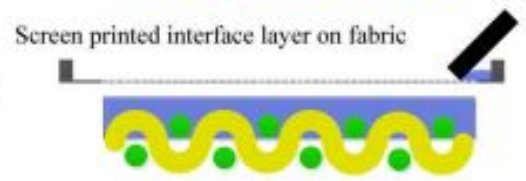

(e)

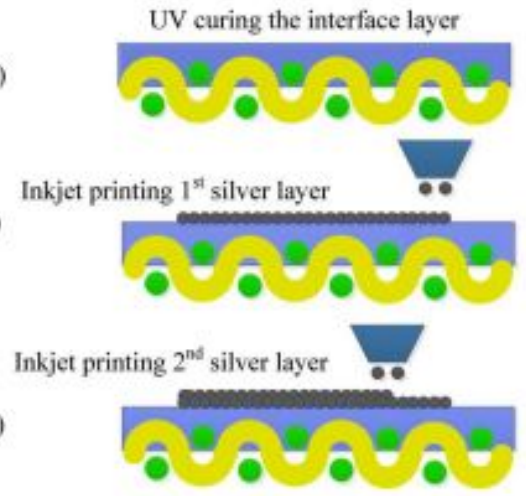

Thermally cured conductive silver layer on fabric

(h)

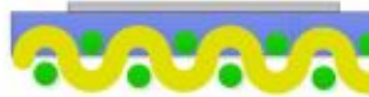

Figure 2.8: Conductive printing on textile using interface layer [1]

The Interface Layer method closely resembles Direct Printing method because it, in the same way, can be easily mass produced, is very similar to PCB production, and it also preserves the relative flexibility of textile. But the main advantage of an interface layer is that it can be used with different materials such as cotton and polyester/cotton that are the two most common textiles available. Additionally, interface layer protects conductive ink layer against excessive bending and crumbling. 
Interface layer can be selectively formed only on regions that are intended for future

conductive ink printing, thus contributing to textile elasticity and breathability [1]. The one drawback of the Interface Layer method is that, despite providing the high degree of textile flexibility, the interface layer still slightly stiffens the textile surface comparable to the rigidity formed by graphic prints.

In short, interface layer method is suitable for Smart Fabric production because it combines easy mass-production characteristics inherent to electronics industry with the high degree of flexibility natural to textiles that can be applied to any fabric material.

\subsubsection{Existing Applications of Interface Layer Method}

Researchers from University of Southampton performed considerable work related to printing via the Interface Layer method. However, they have not accomplished any sophisticated circuit level designs involving integration of electronic components onto textile. Existing applications of interface layer method include antennas dipole(Fig 2.9(a) and patch(Fig 2.9(b) , MEMS structures - valveless micropumps(Fig[2.9(c) and cantilever structures(Fig 2.9(d)], flexible capacitors(Fig,2.9(e)], humidity sen$\operatorname{sors}($ Fig $2.9(\mathrm{f})]$ and the passive electrode network for ECG and EOG applications (Fig 2.1(a)]. 


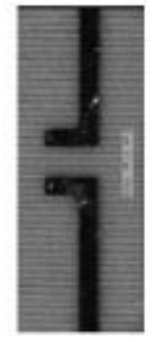

(a) Dipole antenna

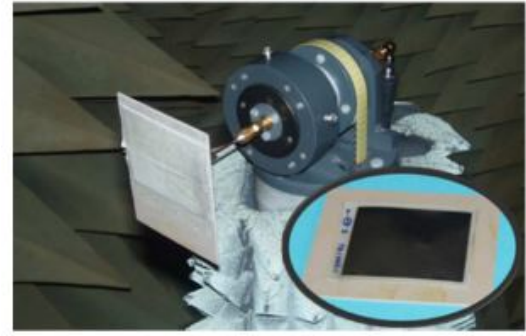

(b) Patch antenna

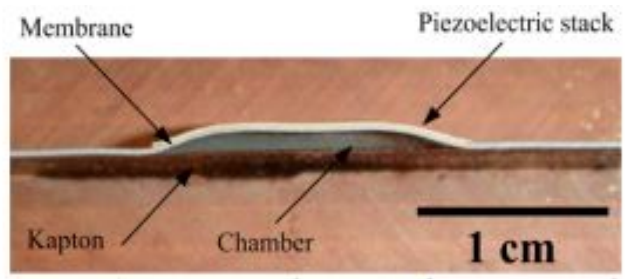

(c) Micropump

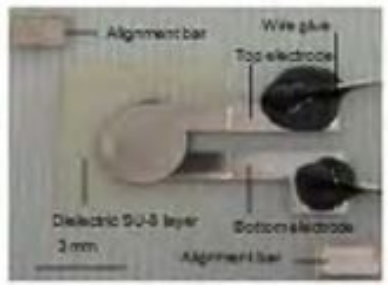

(e) Printed capacitor

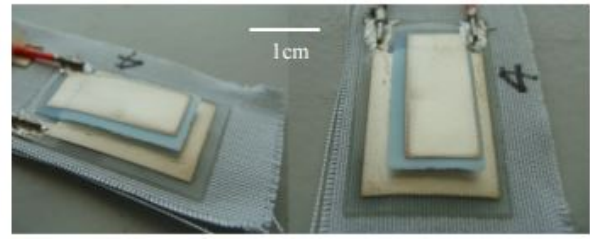

(d) MEMS cantilever

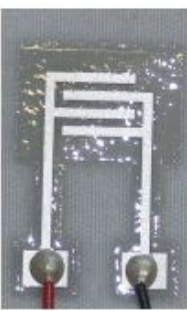

(f) Sensor

Figure 2.9: Existing applications of interface layer method $1-12$

It can be seen from Fig 2.9 that existing interface method applications are of simple structure, mainly interconnects and electrodes connected to supply wires. Even though such elementary devices are of great interest for many applications, they do not demonstrate the full potential of interface layer method, namely building complete circuits with discrete components on clothes for wearable electronic applications. Proving that the interface layer method is suitable for creation of complete working devices will be a considerable step towards PCB-like mass production of flexible Etextiles.

The main ingredient of the Interface Layer method that was used in all previous research papers is a dielectric paste specifically designed to easily adhere to textiles 1- 
12. This dielectric ink is a proprietary interface layer paste Fabink UV-IF4 supplied by Smart Fabric Inks, Ltd (http://www.fabinks.com/). Unfortunately, this interface layer ink is prohibitively expensive - EUR300 for 100 grams as of March 2015. In order to ease the access to the Interface Layer method and decrease the overall cost of prototype production, efforts should be made to adjust a regular low-cost dielectric ink for the application with common textiles.

\subsection{Tracking, Localization or Locating}

Tracking, localization or locating are multiple designations for a useful concept of finding the current position of people, animals and objects. Tracking applications can be found in numerous areas such as military, postal, RFID, mobile, etc. The wearable field is not an exception.

\subsubsection{Tracking in Wearables}

Wearable tracking is an exciting application and is usually accomplished via transmitters, bracelets, watches or ankle monitors Fig 2.10. The main disadvantage of these tracking devices is that they are typically realized as standalone gadgets that a person has to wear or carry around as an extra accessory. Also, such devices are generally bulky, conspicuous and can be lost or forgotten.

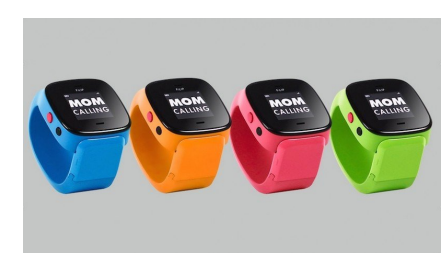

(a) Wearable watches

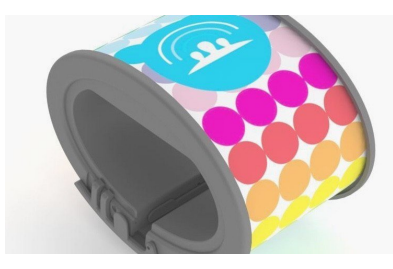

(b) Wearable bracelet

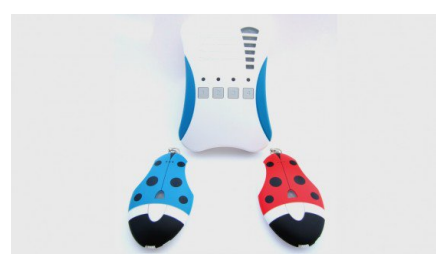

(c) Wearable transmitter

Figure 2.10: Multitude of wearable tracking devices [17

Combining tracking applications with E-textiles solves the additional accessory 
problem. However, currently there are no true E-textiles tracking implementations. A few examples of clothes with tracking features are realized by gluing, stitching or pocketing regular trackers onto textile [57]. Such incorporation of trackers can hardly be labeled as E-textiles. Since these trackers are solid PCB-based, resulting clothes implementation feels bulky and rigid. Moreover, since the incorporation of cumbersome tracking devices on T-shirts or dresses is not feasible, such tracking devices are usually featured on heavy duty clothes such as protective uniforms, coats and ski jackets [48] where the size and rigidness of trackers are of lesser concern.

True E-textiles implementation can potentially be accomplished via conductive printing. Printing on textiles renders PCBs redundant. Utilizing the Interface Layer method, a complete circuit layout can be printed on textile followed by an attachment of discrete components, subsequently making the resulting tracking device much more flexible, inconspicuous, applicable to different clothes types, and in accord with inherent textile flexibility. To the author's best knowledge, there are no E-textile tracking devices implemented via conductive printing so far. Thus, this field is still wide open to the research implementations.

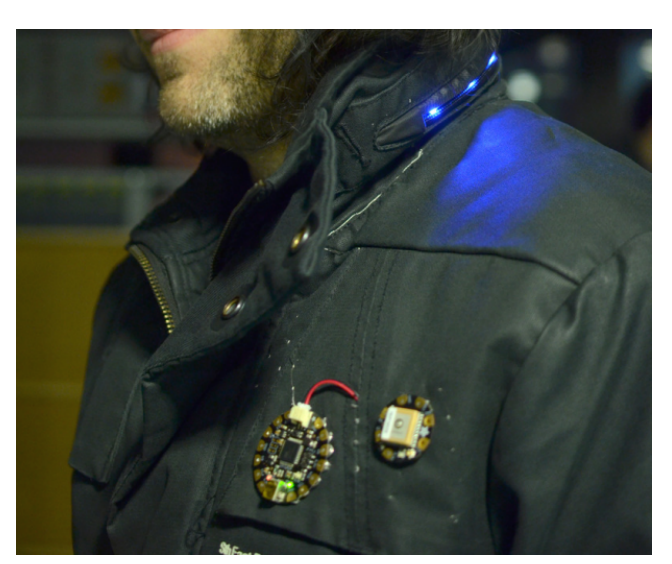

(a) Adafruit's Flora GPS jacket 57

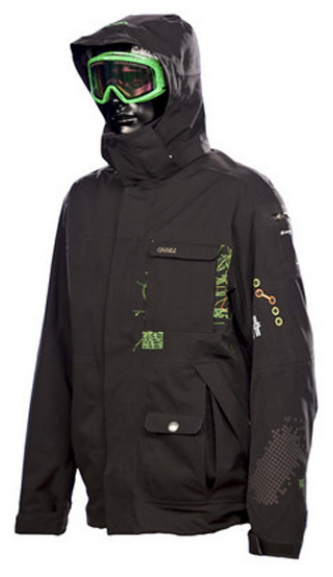

(b) GPS ski Jacket 48

Figure 2.11: Tracking gadgets on ordinary clothes. 


\subsubsection{Tracking Techniques}

Tracking applications are usually realized using one or any combination of Global Positioning System (GPS), Global System for Mobile (GSM) or WiFi-based Positioning System (WPS) localization techniques. However, these techniques have certain limitations when used with E-textiles.

Global Positioning System (GPS) is a well-established localization technology that employs triangulation from satellites that orbit around the Earth. GPS technology is mature and reasonably accurate. Unfortunately, due to GPS frequency of $1.575 \mathrm{GHz}$ and the long transmission distances, antennas suitable for GPS communication tend to be considerable in size and composed of non-printable materials such as ceramics that make GPS antennas poor candidates for printing on textiles. A typical compact ceramic GPS antenna is represented on Fig $2.12(\mathrm{a})$. For better reception, GPS antennas should be faced upwards, thus limiting suitable locations of antenna on clothes. Moreover, GPS technique usually demonstrates decreased tracking capabilities when inside or near big buildings. And in modern settings people tend to spend a lot of time indoors.

Global System for Mobile (GSM) technology uses nearby mobile base stations to triangulate the position. GSM technology is mostly used in mobile phones industry. The main drawback of using GSM technology with E-textile is the obligatory connection to a mobile network that implies the use of SIM-cards (Fig.2.12(b)]. Even without mandatory mobile payments, embedding SIM-card support structure onto textile unnecessarily complicates the circuit and decreases textile flexibility. 


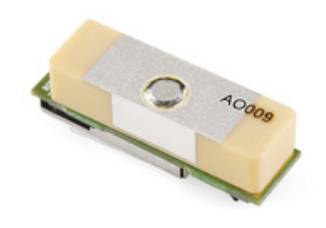

(a) GPS antenna [58]

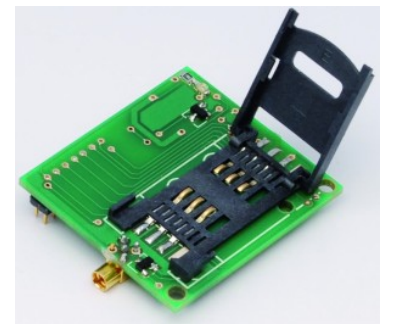

(b) GSM Sim card 59

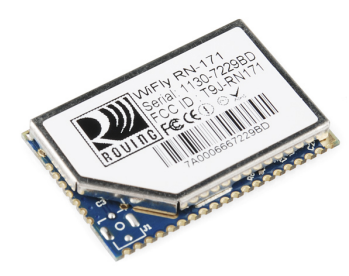

(c) RN171 WiFi Chip

Figure 2.12: GPS, GSM and WiFi

WiFi-based Positioning System (WPS), the term coined by Skyhook Wire [60], determines the location using signal intensity received from nearby wireless access points. WiFi localization has seen the significant growth due to the ever-increasing number of WiFi access points available. WiFi technology is well-suited for E-textiles tracking applications. Due to WiFi $2.4 \mathrm{GHz}$ frequency and relatively short communication distance, WiFi antennas are small, proven to be printable [61] and not heavily affected by the antenna location on clothes. WiFi modules are relatively cheap, compact and continuously decreasing in size (Fig.2.12(c)). WPS accuracy depends on the number of access points entered into WPS database. Since this database is continually being complemented and new WiFi localization algorithms are being developed [62 65], the locating accuracy is constantly increasing [60]. The main limitation of WiFi-based localization is the actual presence of a WiFi signal since it is needed to connect with access points database. But this limitation is dwindling due to rapid global WiFi integration.

\subsection{Conclusion}

E-textiles can be fabricated using different methodologies. Table 2.1 reports characteristics comparison of major fabrication techniques described in this Chapter. The comparison is made according to three characteristics - flexibility, mass production 
and material. Flexibility indicates whether manufacturing method keeps E-textile's elasticity relatively intact. Mass production points out if the manufacturing method is capable of efficient volume production. Material characteristics state whether manufacturing method applies to most of the textile materials.

Table 2.1: Characteristics Comparison of E-textiles manufacturing methods

\begin{tabular}{|l|c|c|c|}
\hline Method & Flexibility & Mass Production & Material \\
\hline Direct attachment & No & Yes & Yes \\
\hline Application of conductive foil & Yes & No & Yes \\
\hline Sewing, embrodering, knitting, etc. & Yes & No & Yes \\
\hline Direct conductive printing & Yes & Yes & No \\
\hline Interface layer method & Yes & Yes & Yes \\
\hline
\end{tabular}

The Interface Layer method scores in all three categories - it preserves textile flexibility, it can work with different textile materials and it can be easily mass produced using ink-jet or screen printing techniques. Besides, circuit layouts generated by the Interface Layer method are very similar to the ones produced by traditional PCB manufacturing.

However, current research of the Interface Layer method lacks in volume and only simple structures consisting of lines, patches and electrodes were studied. The feasibility of utilizing an interface layer to create a complex circuit layout on fabric needs to be addressed. The best way to check the feasibility is to print an actual circuit on fabric using the Interface Layer method.

It has been shown that there are no true E-textile tracking devices currently present on the market. The only applications are those featuring separate GPS trackers that are attached to heavy clothes such as uniforms and ski jackets. The Interface Layer method is the perfect candidate to realize the first E-textile tracking device. Such devices featuring WiFi-based localization will be flexible and mass producible. Being able to successfully produce the on-textile localization device will achieve two major goals, namely validate the Interface Layer approach as a suitable E-textiles 
production method, and create the first true E-textiles localization device. Reaching these goals will lay down a strong foundation for future development of E-textiles via Interface Layer method and will open the path for realization of diverse applications such as Internet of Things (IoT). 


\section{Chapter 3}

\section{The Interface Layer Method:}

\section{Requirements, Fabrication and}

\section{Characterization}

The Interface Layer method is a favorable approach for the production of E-textiles. As it was discussed in Chapter 2, among different E-textiles manufacturing technologies, only the Interface Layer method is suitable for volume production of Smart fabrics from any textile material while preserving the natural elasticity of the clothes. The Interface Layer method has been successfully used for printing of simple structures such as lines and electrodes on textile. However, it is the printing of a complete circuit layout, followed by incorporation of discrete electronic components on fabric, that can ultimately prove that an interface layer method is suitable to act as the bridge between traditional electronic manufacturing and clothing production industry. The resulting device, incorporated on fabric using the Interface Layer method, will be both relatively flexible similar to ordinary clothes and easily mass producible similar to PCB electronic production. In order to construct such device, the interface layer formation should be thoroughly studied. In this chapter, requirements, fabrication and characterization of the Interface Layer method are discussed. 


\subsection{Interface Method Requirements}

In order to perform the successful conductive printing via the Interface Layer method several arrangements should be made - fabric substrate and interface dielectric material should be identified, suitable dielectric paste application process should be outlined, and subsequent conductive printing process summarized.

\subsubsection{Interface Layer Material Selection}

The only interface layer ink that has been featured in previous research papers related to the Interface Layer method is the Fabink-UV-IF4 UV curable polyurethane acrylate based interface paste $1-12]$. This dielectric paste was specifically designed to be used with various textile substrates. More details regarding Fabink-UV-IF4 ink can be found in Table 3.1. This dielectric paste has already been successfully used by researchers from the University of Southampton to create interface layers; however, its purchase cost is prohibitively expensive (EUR300 for 100g) which impedes the progressive development of the conductive printing field. In this chapter, Fabink-UVIF4 will be utilized to formulate the sample interface layer fabrication process that later will be used as the reference to optimize the interface layer formation using a general purpose dielectric ink.

The Creative Materials 116-20 (CM 116-20) is a general purpose UV-curable dielectric ink suitable for various applications including flexible circuits. This ink is supplied by Creative Materials (http://www.creativematerials.com/) and is seven times cheaper than the Fabink-UV-IF4. More details regarding CM 116-20 can be found in Table 3.1. The Interface Layer method is a relatively new field. To the author's best knowledge, there is currently only one research group that practices this method and they performed no attempts to employ any other interface layer paste besides Fabink-UV-IF4. No other dielectric inks have ever been researched as suitable inter- 
face layer candidates. However, the employment of a general purpose dielectric such as CM116-20 ink for interface layer formation will substantially decrease the production cost that in turn will facilitate the Interface Layer method research as well as the whole E-textile field. Cheaper interface layer material will lower the entry barrier for prototyping of E-textiles applications.

Since no general purpose dielectric paste has ever been used for the interface layer formation, there is no research that outlines the interface layer manufacturing steps via the CM116-20 dielectric paste. Thus, this is still an uncharted territory. As it was mentioned before, later in the chapter, the interface layer formation recipe for FabinkUV-IF4 will be formulated. However, it is expected that this recipe will not work for the CM116-20. Therefore, the Fabink-UV-IF4 recipe will be used as a starting point to develop the optimal interface layer formation recipe for the CM116-20 dielectric paste. Eventually, two interface layers, one from the specially designed interface paste Fabink-UV-IF4 and another from the general purpose dielectric ink CM 116-20, will be analyzed against each other.

Table 3.1: Fabink-UV-IF4 and CM 116-20 Data

\begin{tabular}{|l|c|c|}
\hline Properties & Fabink-UV-IF4 & CM116-20 \\
\hline Rheology: & screen printable paste & screen printable coating \\
\hline Cure Method: & UV 30sec at $1500 \mathrm{mj} / \mathrm{cm}^{2}$ & UV 1min. at 300 watt/inch \\
\hline Color: & Clear & Clear \\
\hline Compatibility: & textiles, alumina, Kapton & polycarbonate, Kapton, epoxy/glass \\
\hline Cost per gram: & USD 3.5 & USD 0.5 \\
\hline
\end{tabular}

Fig 3.1 features Fabink UV-IF4 costing EUR 300 per 100 gram jar on the left side and Creative Materials 116-20 costing USD 250 per 473 gram jar. 


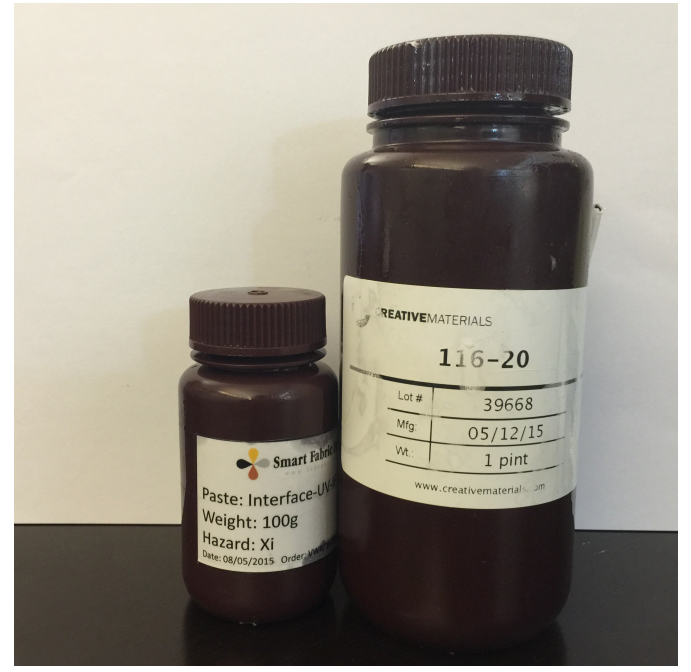

Figure 3.1: Dielectric inks - Fabink-UV-IF4 on left side, Creative Materials 116-20 on right side

\subsubsection{Fabric Selection}

Even though the Interface Layer method is potentially suitable for any textile material, the fabric selection still matters because it affects the electromagnetic properties of a printed antenna that is a crucial part of any localization device. For this research three common woven fabric substrates were selected - 100\% cotton, $65 \% / 35 \%$ polyester/cotton and 85\%/15\% polyester/cotton (Fig.3.2). These textile substrates are very commonly used. $100 \%$ cotton is the most common T-shirt material. $65 \% / 35 \%$ polyester/cotton is used to make shirts. Finally, 85\%/15\% polyester/cotton is used for protective clothing such as lab coats.

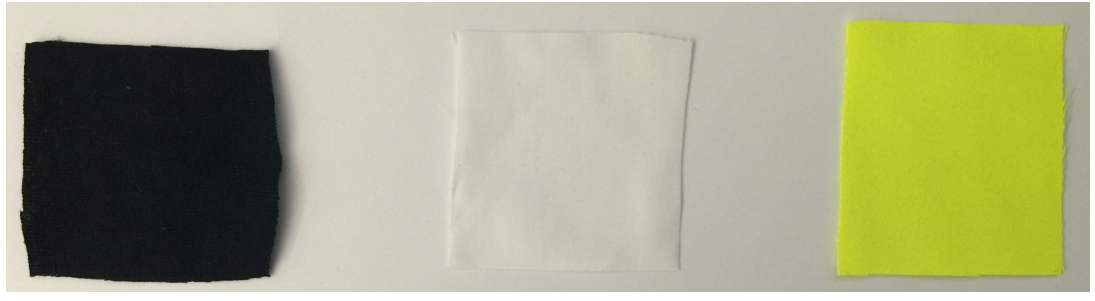

Figure 3.2: Textile substrates $100 \%$ cotton (left), $65 \% / 35 \%$ polyester/cotton (middle) and $85 \% / 15 \%$ polyester/cotton(right). 
In order to prove that the Interface Layer method can work with any standard textile material regardless of its characteristics, $100 \%$ cotton and $65 \% / 35 \%$ polyester/cotton fabrics were purchased from general purpose clothing department stores in Jeddah, Saudi Arabia. Therefore, their physical properties such as density and weave are unknown. Nevertheless, interface layer surface roughness and electrical characteristics of these textiles will be investigated. On the other hand, 85\%/15\% polyester/cotton textile with the brand name "Luminex 310" was purchased from Klopman International (http://www.klopman.com/); therefore, the weave type and density are known and shown in Table 3.2. This textile material will be used as the final circuit's substrate because its exact supply source and properties are known; thus, the circuit design can be easily repeated and/or validated by other interested researchers. Due to the same reason $85 \% / 15 \%$ polyester/cotton textile will be used as the substrate for the comparison of the special interface paste Fabink-UV-IF4 and the general purpose Creative Materials 116-20 dielectric ink.

Table 3.2: Properties of textile substrates

\begin{tabular}{|l|c|c|c|c|}
\hline Material & Density & Weave & Collor & Source \\
\hline $100 \%$ cotton & Unknown & Unknown & Black & Regular Store \\
\hline $65 \% / 35 \%$ polyester/cotton & Unknown & Unknown & White & Regular Store \\
\hline $85 \% / 15 \%$ polyester/cotton & $310 \mathrm{~g} / \mathrm{m}^{2}$ & Satin & Yellow & Klopman International \\
\hline
\end{tabular}

\subsubsection{Interface Layer Application Method}

Screen printing is the method that has already been used in electronic industry to apply conductive and dielectric inks onto various substrates such as Kapton, Mylar, glass, polycarbonate, treated and untreated polyesters, glass, epoxy PC boards, etc. Furthermore, screen printing is a well-established and widely used technique to print arts, graphics and designs on fabric, for example on T-shirts. Due to its reel-to-reel and roll-to-roll capabilities screen printing is ideally suitable for mass production. However, when it comes to E-textiles, the screen printing is still in its infancy. Being 
applicable to both electronic manufacturing and textile industry, screen printing, with proper research, has a potential to become a production method of choice for printing circuit layouts on textiles. Standard screen printing procedure is explained in Fig 3.3

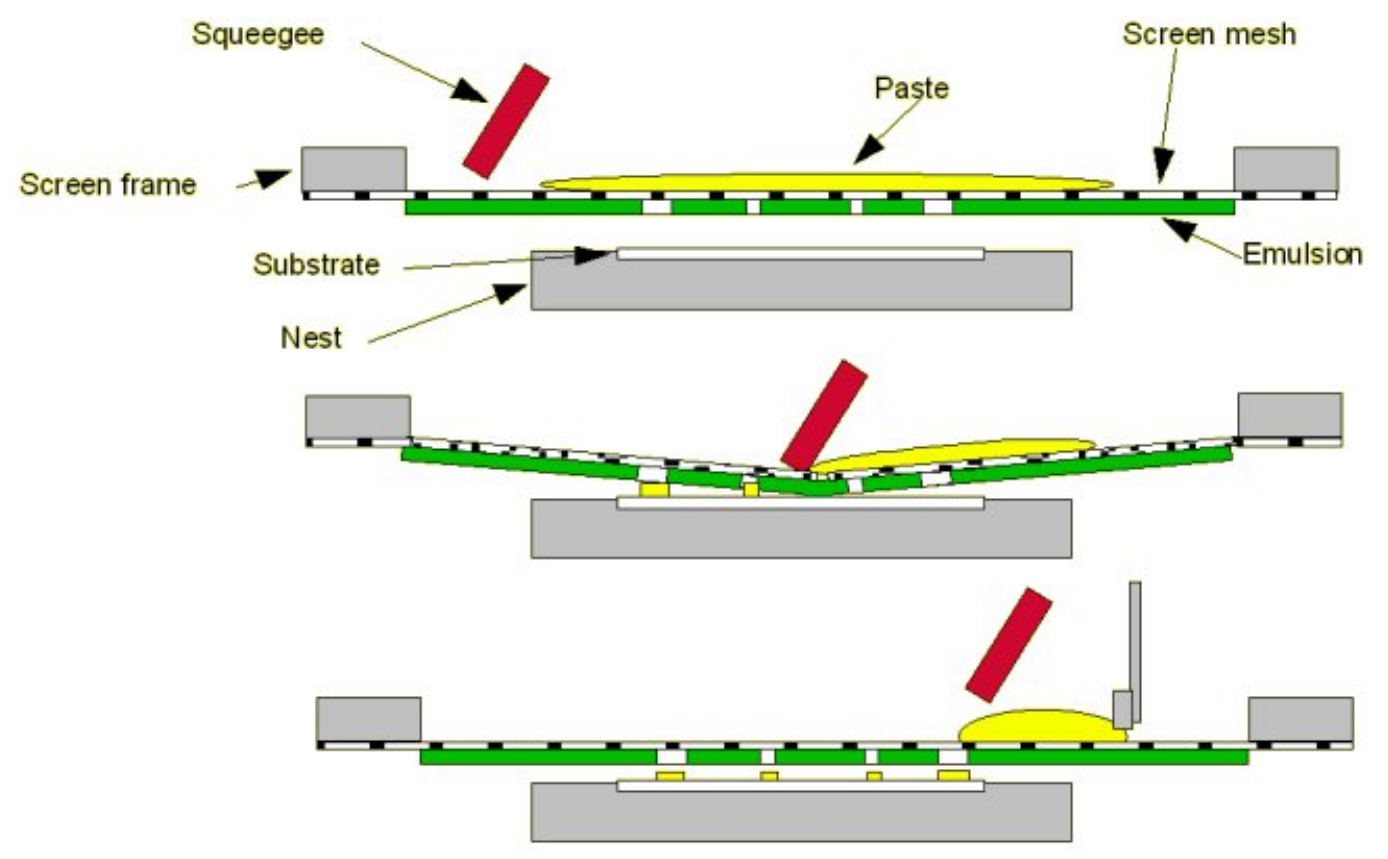

Figure 3.3: Common screen printing process [18]

Regular screen printing process involves a screen, paste, substrate and squeegee. The screen consists of a hard frame, a screen mesh and an emulsion that seals the screen mesh in regions were no printing should occur, thus creating a printing pattern. The screen is placed above the substrate that is rested on the nest that is just a flat surface. The squeegee is used to push paste through the screen mesh in areas that are not covered by emulsion. Paste that is pushed through, forms the desired pattern. Later, the deposited paste should be UV or thermally cured.

In this work, the manual screen printing, also called "Doctor Blading", is employed. The "Doctor Blading" technique is conceptually similar to the screen printing process described above, but, instead of an automatic or semi-automatic machine movement, the squeegee is slid manually. This method is not as accurate as the 
standard screen printing, but it is much more flexible and faster which is great for prototype development. The main drawback of the manual screen printing is that the deposited layer tends to be thicker than the one produced by the automatic or semi-automatic screen printing where it is possible to control precisely the deposition thickness. However, "Doctor Blading" technique is more than enough to achieve a reasonably smooth and uniform dielectric layer suitable for the use with the Interface Layer method. And, if a fully-functional tracking device can be created using the "Doctor Blading" technique, it a fortiori can be produced using the more precise standard automatic or semi-automatic screen printing technologies.

In this research, for screen printing purposes, the screen was replaced by a PET film. Such change allows for increased flexibility since professional screens come with preset designs, and it takes considerable time to order screens with a new design even if a slight change in the print pattern is required. Professional screens are great when the design is already well-established, and the screen will be used for mass production. But for prototyping purposes professional screens are too unadaptable because any pattern alteration requires a new screen. Contrarily, PET film stencils can be easily and quickly altered using laser-cutting or simple manual carving. Also, the deposition thickness can be roughly adjusted by stacking together additional PET films. Three PET layers of the total thickness of $0.3 \mathrm{~mm}$ were experimentally found to produce adequate interface layer formation results. This stencil thickness will be used in all interface layer printings. The three-layer PET stencil with a simple rectangular pattern will be used for prototyping (Fig $3.4(\mathrm{a})$. The squeegee used is the professional grade D-cut with plastic blade represented on Fig $3.4(\mathrm{~b})$. The dielectric paste is the Fabink-UV-IF4 and CM116-20 shown on Fig.3.1 and the substrate is the 85\%/15\% polyester/cotton fabric textile shown on Fig. 3.2 . 


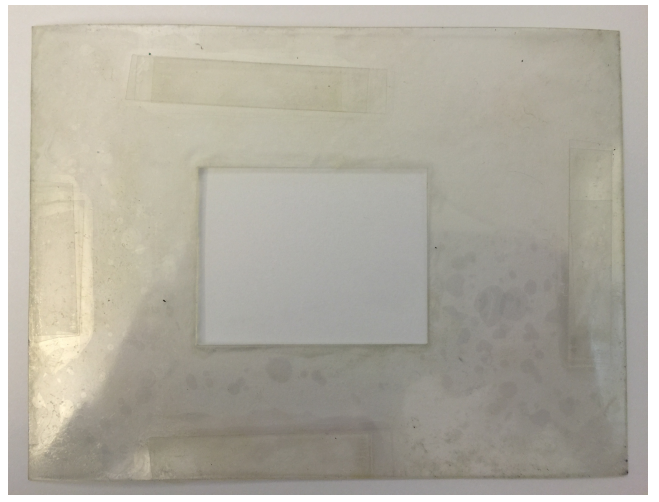

(a)

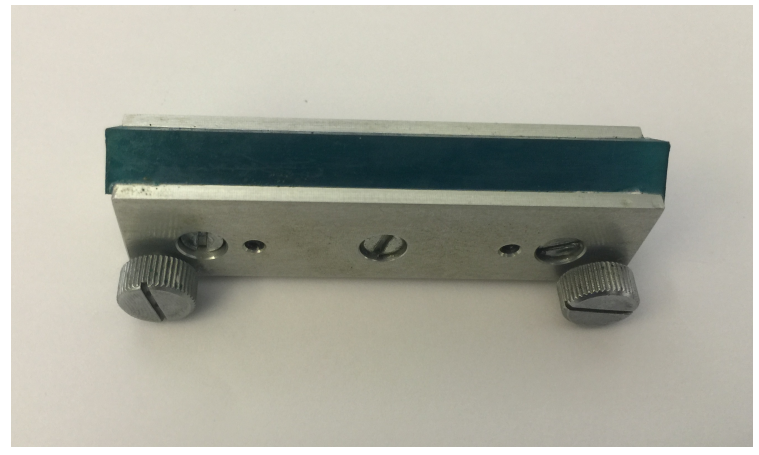

(b)

Figure 3.4: Screen printing accessories: a) 3 layer PET stencil b) D-cut squegee

The manual screen printing is commenced by placing a textile sample on a flat surface and then placing the PET stencil on the textile. The sufficient amount of dielectric ink is poured on the stencil side. After the application, ink remainders can be gathered back into the bottle. A squeegee is used to scrape dielectric ink across the stencil surface. The application is done in two strokes - forth and back while maintaining constant force and blade angle. The manual screen printing process is presented on briefly in Fig 3.5 .

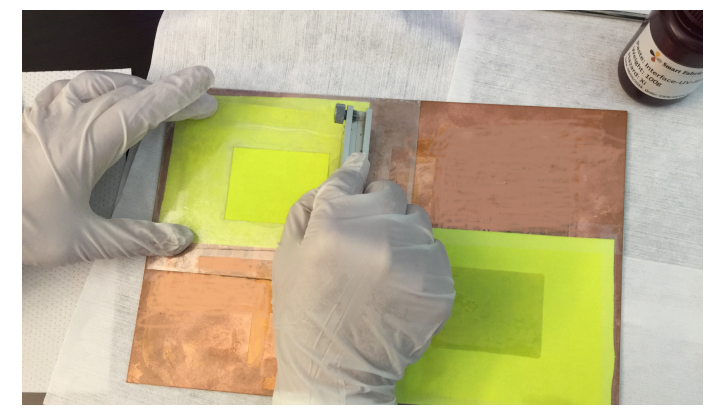

(a)

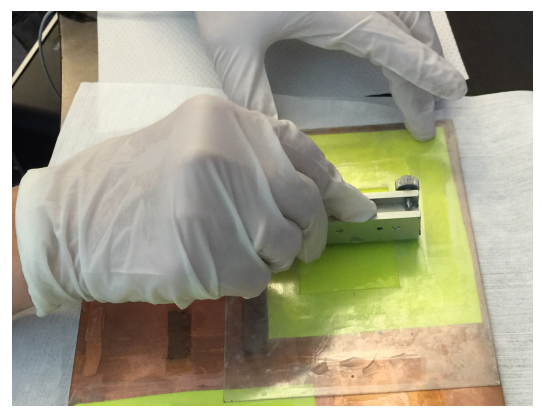

(b)

Figure 3.5: Manual screen printing process: a)top view b)side view 


\subsubsection{Conductive Printing}

Conductive printing is accomplished either by screen or ink-jet printing. Conductive layer screen printing follows the same procedure as the screen printing described in section 3.1.3, but, instead of a dielectric paste, a conductive paste is used. Conductive screen printing, once again, is perfect for printing established patterns in bulk. However, in this work, conductive printing will be accomplished using the ink-jet printing technique, because it is more suitable for prototyping since it is rapidly adjustable and easy to set up. Also, ink-jet printing is all digital and does not require a screen to operate. The ink-jet printing is accomplished via special ink-jet printer. For this research work, Dimatix DMP 2831 printer, shown on Fig,2.5(a), is used. This printer utilizes one and ten picoliter cartridges to deposit serially conductive drops onto the substrate in a predefined pattern. Ten picoliter cartridges are used in this research. The distance between drops is critical to establish a good structure conductivity. The drop spacing of 20um was experimentally found to perform well providing sharp line edges and almost no side overspills. The silver nanoparticle ink DGP 40LT-15C that is provided by Advanced Nano Products (http://anapro.com/) was used as the conductive ink. After printing, the deposited layers were each sintered for 20 mins at $130^{\circ} \mathrm{C}$ in a thermal oven. The chosen curing process is suggested by ink manufacturer and also can be safely used with textile substrates selected in section 3.1.2. The printing process is represented on Fig.3.6. 


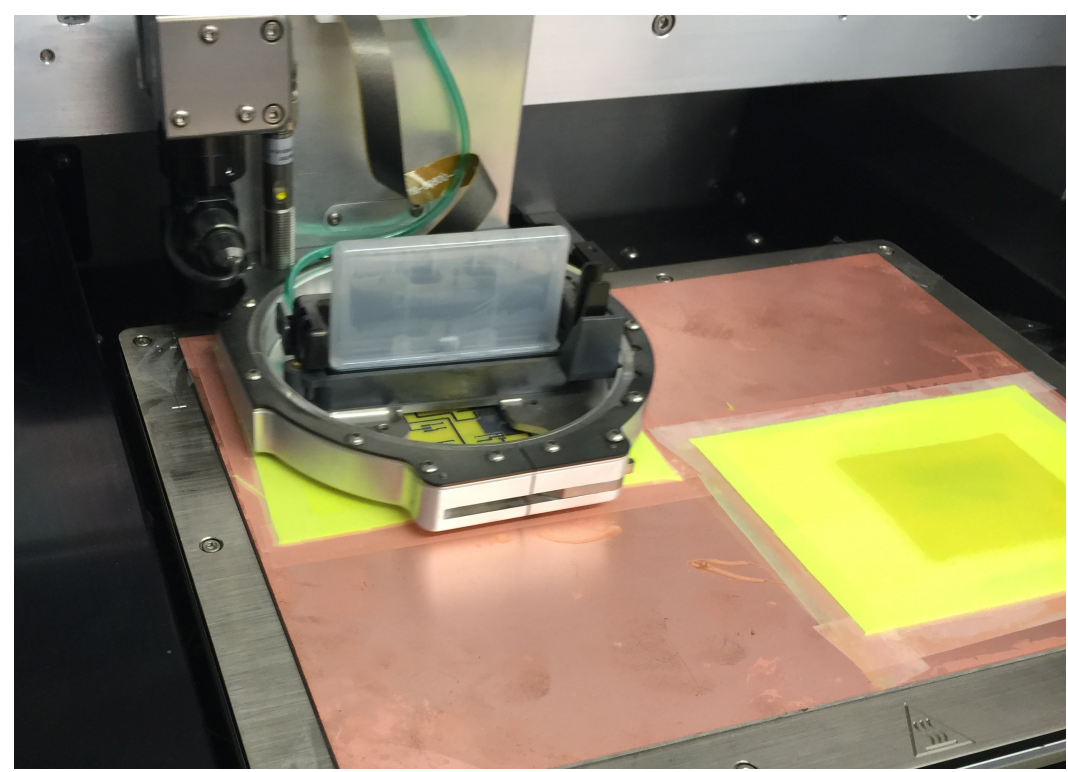

Figure 3.6: Ink-jet printing procedure

\subsection{Interface Layer Fabrication}

The interface layer formation is an entirely additive process that flattens the textile surface allowing for printing. In this research, the interface layer is deposited via the manual screen printing method. This section describes the interface layer formation processes using two dielectric inks - special interface layer paste Fabink-UV-IF4 and general purpose dielectric ink CM116-20. Two interface layers are created, and comparison has been performed. $85 \% / 15 \%$ polyester/cotton textile was used as the substrate material. The three-layer PET film of $0.3 \mathrm{~mm}$ combined thickness was used as the stencil. Simple rectangle $65 \times 50 \mathrm{~mm}(\mathrm{WxH})$ was selected as the pattern(Fig.3.4(a)). It is worth noting that any pattern shape can be used for the interface layer formation.

\subsubsection{Fabink-UV-IF4}

The Fabink-UV-IF4 ink was specially designed to work with an interface layer material with different textiles. However, its cost is prohibitively high. The interface layer 
formation using Fabink-UV-4 is depicted in Fig 3.7 where following fabrication steps take place:

1. PET stencil is placed above the fabric textile.

2. Fabink-UV-IF4 dielectric paste is poured onto the stencil.

3. Squeegee is used to scrape dielectric paste across the stencil for the first time. Application force and angles should always be constant.

4. In order to achieve the better surface roughness the same procedure as in Step 3 should be performed again but in the opposite direction.

5. Finally, the stencil is removed and the textile substrate is UV cured for $2 \mathrm{~min}$ at $1000 \mathrm{~mJ} / \mathrm{cm}^{2}$ (the cure energy is taken from Fabink-UV-IF data sheet). 


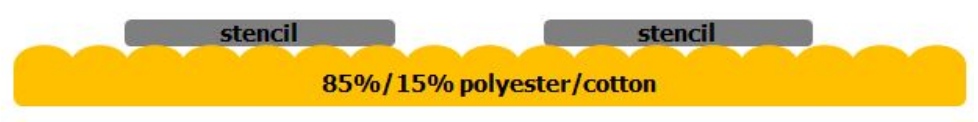

(a)

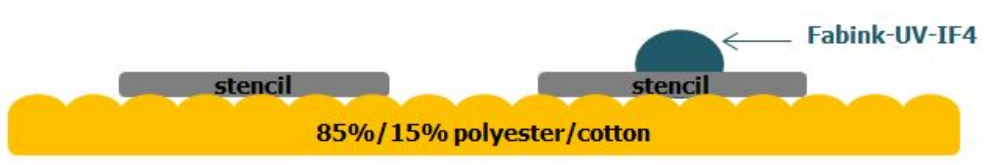

(b)

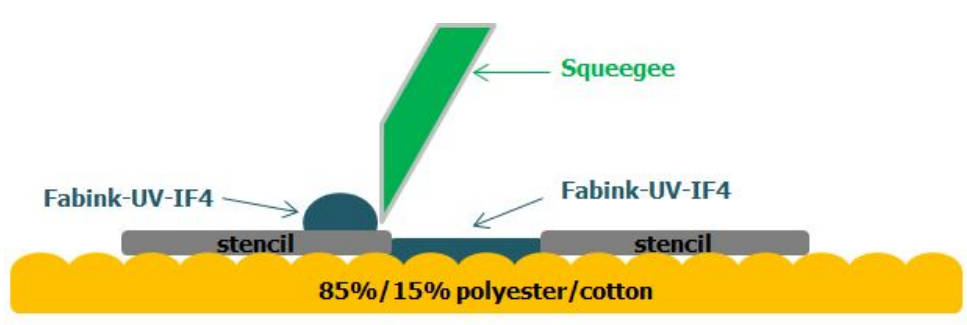

(c)

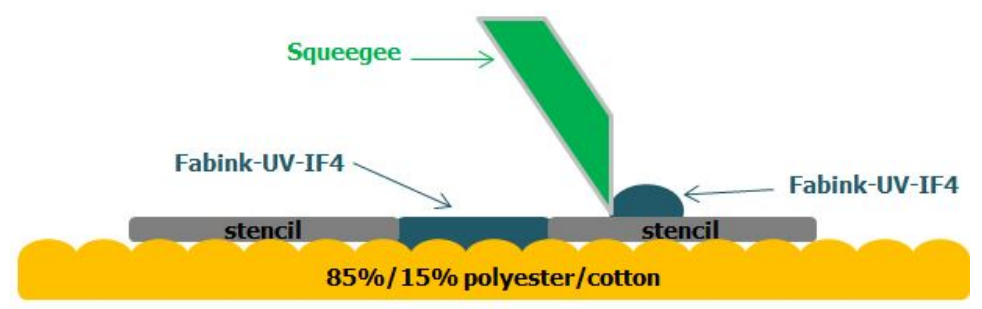

(d)

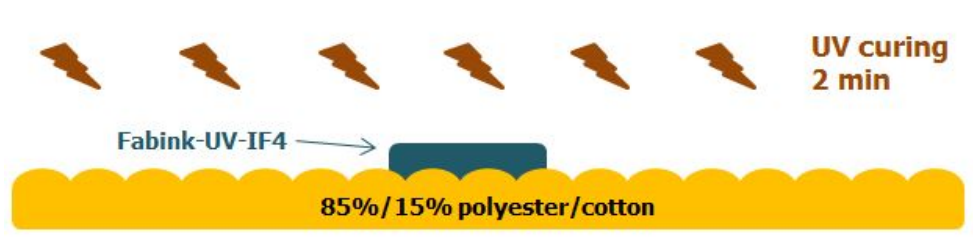

(e)

Figure 3.7: Interface layer formation steps using Fabink-UV-IF4 a) Set up b) dielectric paste application c) first squeegee pass d) second squegee pass e) UV curing

The resulting interface layer is shown in Fig. 3.8 . It can be seen that the layer is clear, uniform and flat, but slight surface roughness can still be observed. Light aureole can be detected around the interface layer rectangle. This happens due to the small leakage of interface ink onto the textile around stencil side edges. Such percolation should not take place during automatic or semi-automatic screen printing 
since the screen will not touch the fabric substrate. Note, this interface layer formation process will be used as the starting point for the interface layer creation via the general purpose CM116-20 dielectric ink.

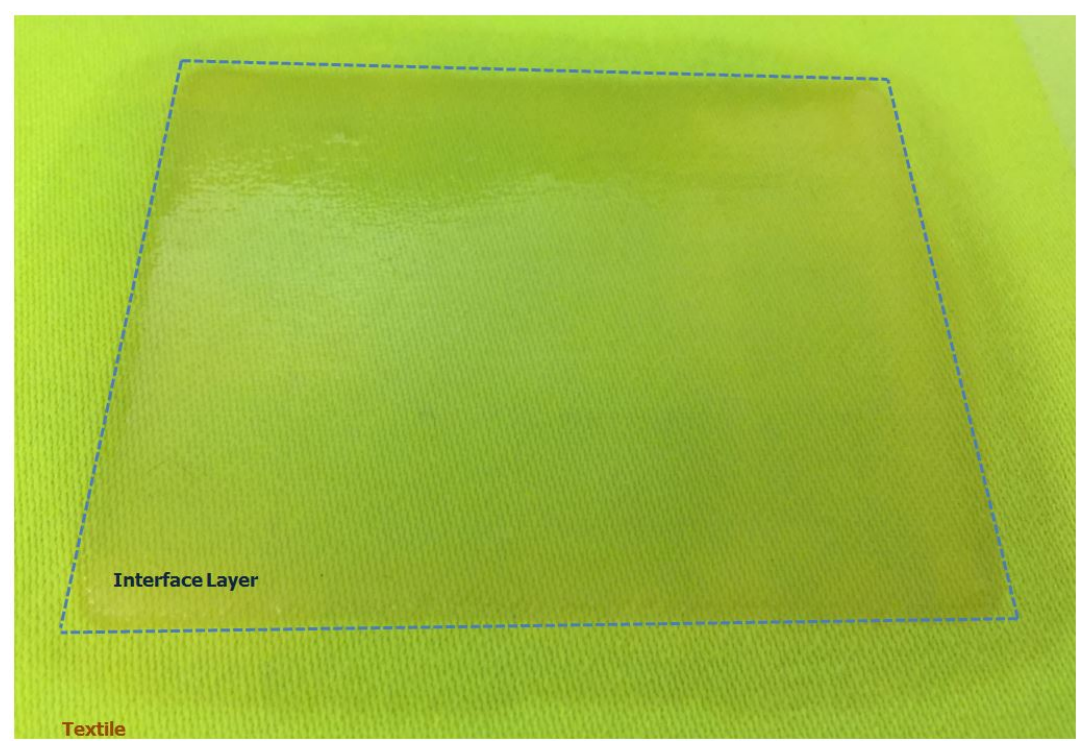

Figure 3.8: The interface layer formed using Fabink-UV-IF4. The dotted line was added for easier visualization.

Conductive ink-jet printing was performed on the Fabink-UV-IF4 interface layer. The resistance and conductivity results were consistent with previous research $[1-12]$.

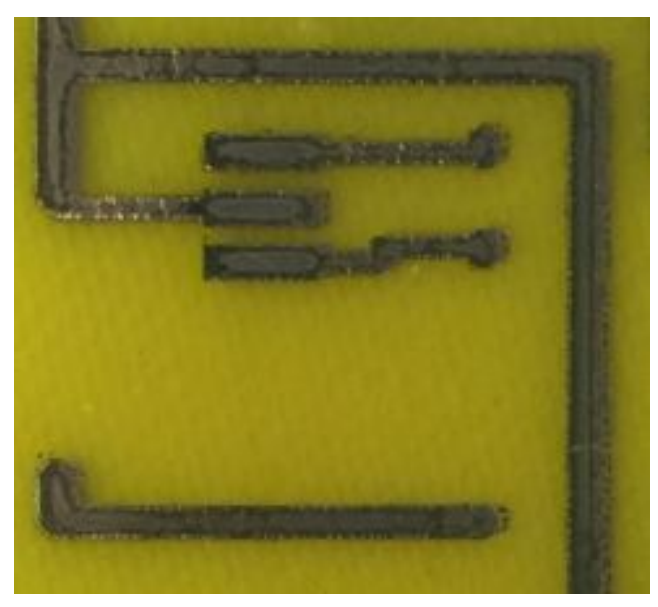

Figure 3.9: Conductive lines printed on the Fabink-UV-IF4 interface layer. 


\subsubsection{CM116-20 Dielectric Paste}

The Creative Materials 116-20 ink is a general purpose UV-curable dielectric paste. This paste was not specifically designed for textile materials. However, its cost is much less compared to the cost of Fabink UV-IF-4. When performing the same fabrication steps described in section 3.2.1 utilizing the CM116-20 dielectric ink, the interface layer is produced, but it is not suitable for conductive printing. When attempting to ink-jet print, heavy diffusion of conductive ink into the interface layer occurs (Fig 3.10). Due to this diffusion, silver nanoparticles fail to form a uniform conductive layer during the sintering step.

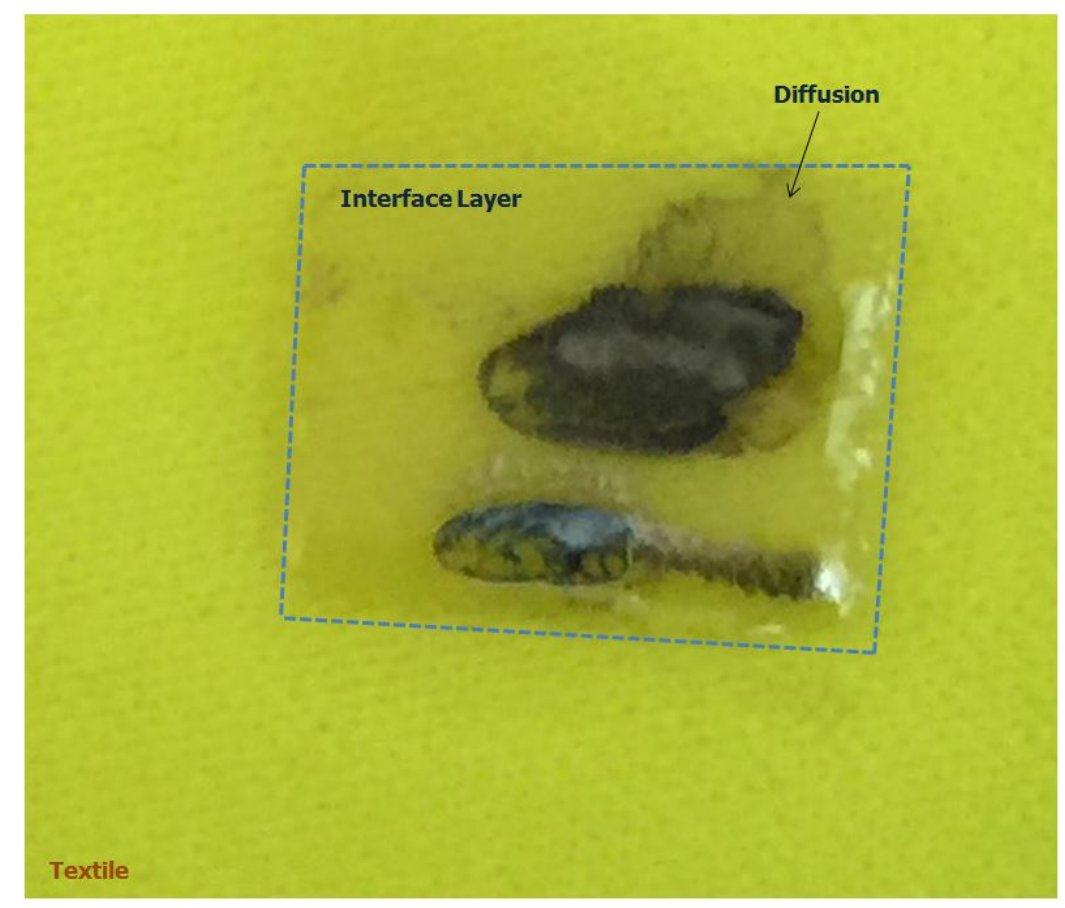

Figure 3.10: Diffussion of conductive ink into CM116-20 interface layer. The dotted line was added for easier visualization.

As it was expected, the interface layer fabrication process described in section 3.2.1 did not work for the general purpose CM116-20 ink and resulted in the massive diffusion of conductive ink into the interface layer. The diffusion takes place because the underlying CM116-20 interface layer is not completely UV-cured. This observa- 
tion is supported by the fact that even after prolonged UV treatment, the interface layer is not completely solidified and feels slightly viscous. The incomplete stabilization of the dielectric layer stems from the relatively high interface layer thickness. The CM116-20 ink was designed to be used with automatic or semi-automatic screen printers that are capable of depositing thin layers that are much easier and faster to UV-cure. However, to completely cover the textile surface roughness, the interface layer should be thick enough. Therefore, to use the CM116-20 dielectric paste, the initial Fabink-UV-IF4 interface fabrication process should be modified. There are two possible solutions. The first solution is to decrease substantially the stencil thickness and perform the same interface layer formation depicted on Fig 3.7 , and then marginally increase the stencil thickness and perform the interface layer formation procedure again, and so on. However, this approach is not ideal because it is labor intensive and, due to manual screen printing, it is hard to control accurately the thickness of deposited layers. Another approach is to modify the curing procedure in such way that the CM116-20 interface layer is completely solidified. After several fabrication attempts, curing steps that ensure interface layer solidification were designed and the following optimized interface layer fabrication steps using the general purpose CM116-20 were developed (steps one through four are same as in section 3.2.1):

5. The CM116-20 interface layer is UV-cured for $5 \mathrm{~min}$ at $1000 \mathrm{~mJ} / \mathrm{cm}^{2}$

6. The textile substrate is placed in an oven for 1 hour at $130^{\circ} \mathrm{C}$. During this step a proper ventilation should be ensured. Note, that textiles in this research were tested to withstand such temperature without noticeable degradation of textile qualities. However, textile flammability characteristics should be checked if any other textile material is considered as the substrate for the interface layer printing. 
7. Let the interface layer on textile dry at room temperature for at least 1 hour.

The resulting interface layer formed by the general purpose CM116-20 dielectric ink can be seen on Fig 3.11. The produced interface layer is flat, uniform and clear.

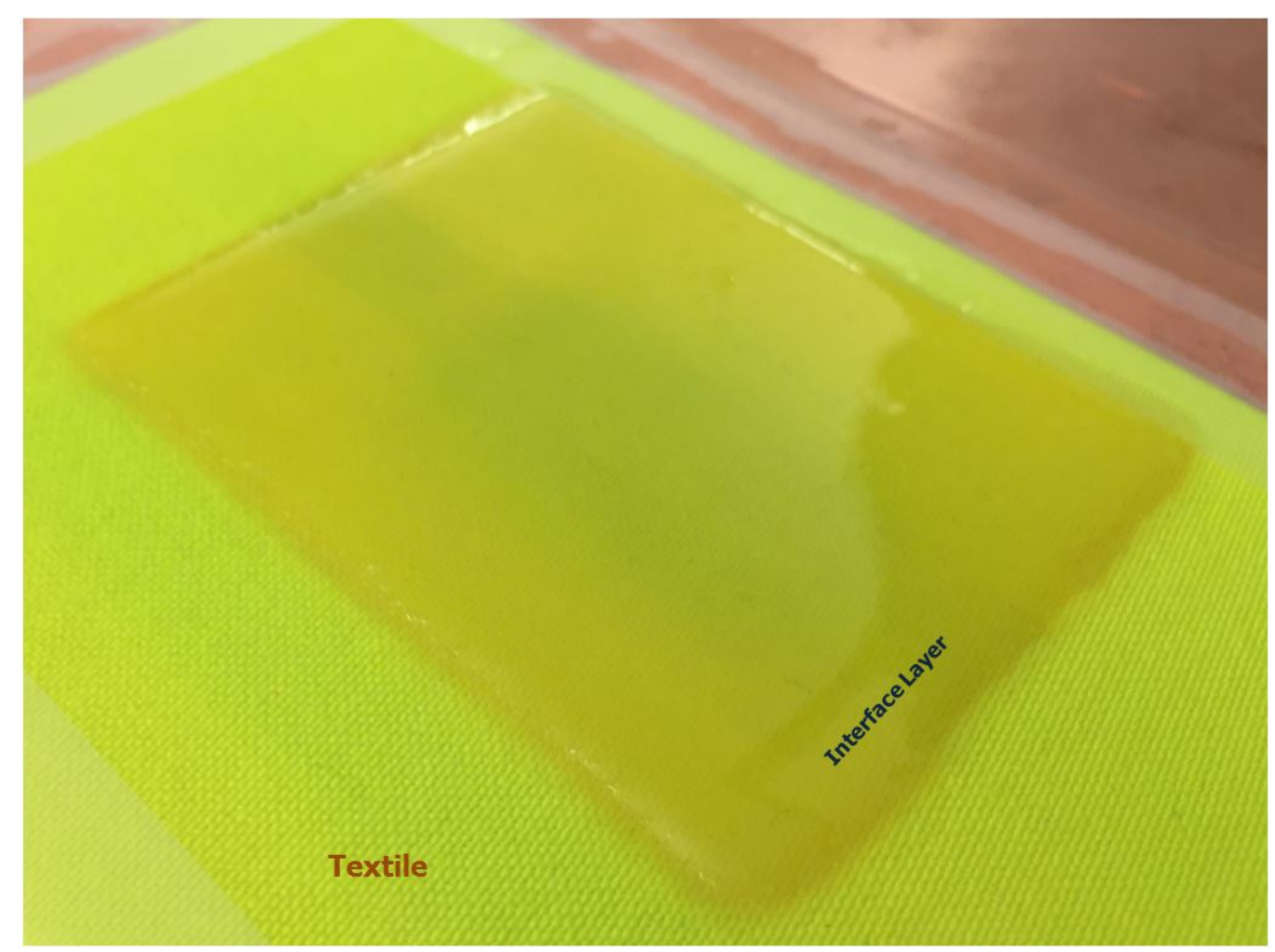

Figure 3.11: The interface layer formed using CM116-20.

\subsubsection{Fabink-UV-IF4 and CM116-20 Comparison}

It has been shown that it is possible to create interface layers using both the special interface layer paste Fabink-UV-IF4 and the general purpose dielectric ink CM116-20. Both interface layers have very similar physical appearances - clear, uniform and flat. However, when compared, the Fabink interface layer appears to be more transparent than the CM116-20 layer that appears yellowish as can be seen on Fig 3.12. Other physical characteristics are very similar. Both interface layers show the high degree of elasticity that is very beneficial for textile applications (Fig 3.12). After bending, 
the interface layers slowly return to the initial flat position. Even after fifty bending cycles similar to the ones done on Fig.3.12, both interface layers do not exhibit any cracking.

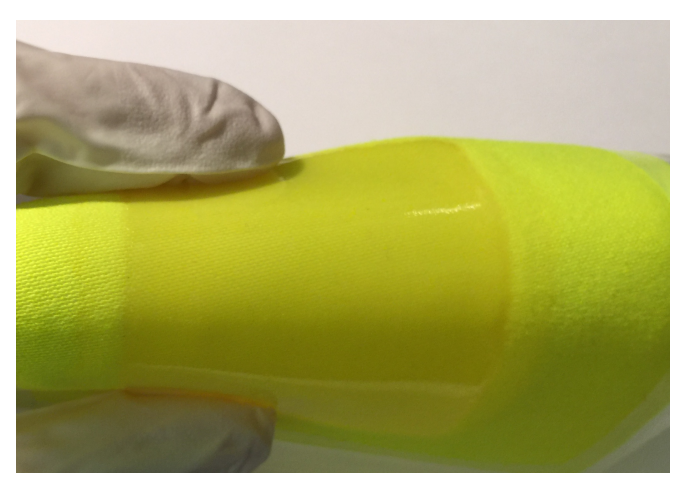

(a)

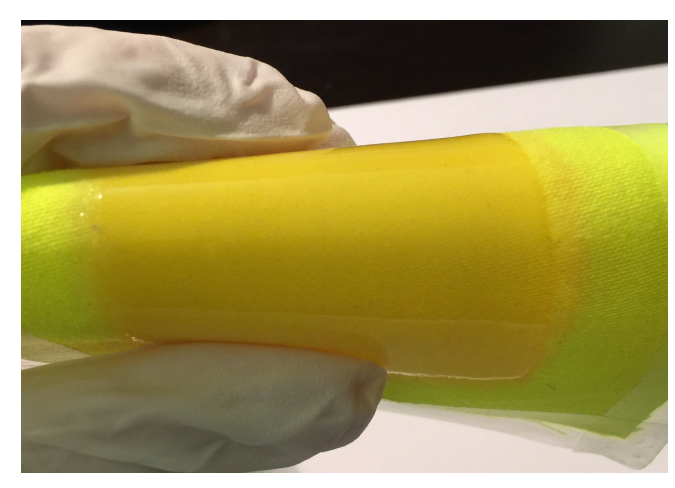

(b)

Figure 3.12: Interface layer bending: a)Fabink-UV-IF4 b)CM116-20

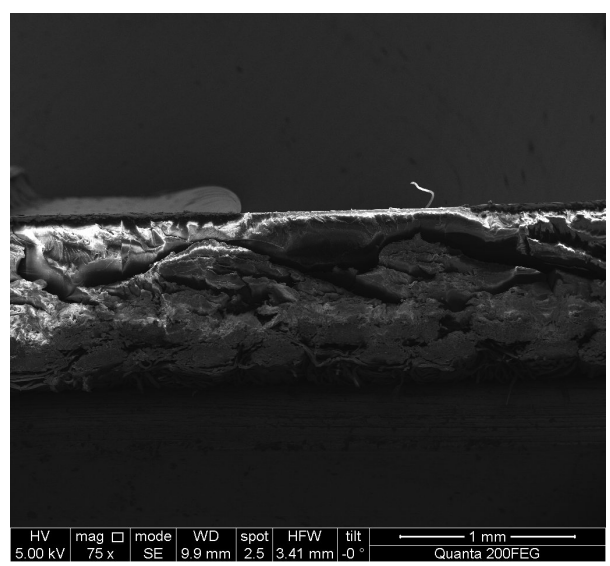

(a)

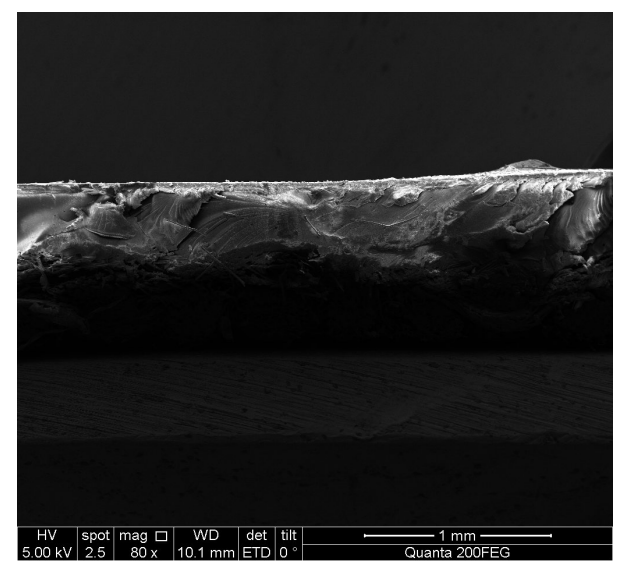

(b)

Figure 3.13: SEM of interface layers: a)Fabink-UV-IF4 b)CM116-20 


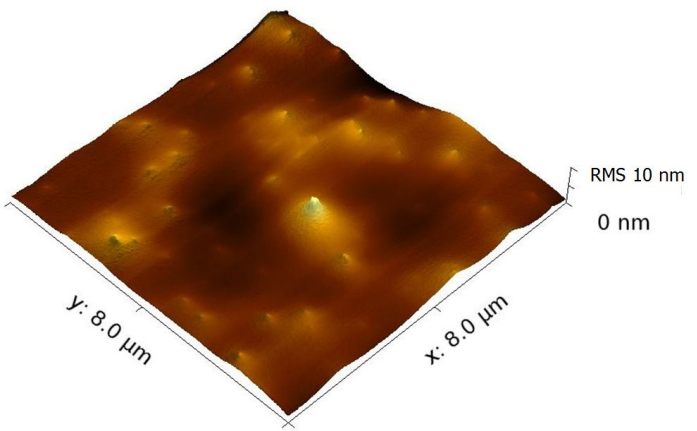

(a)

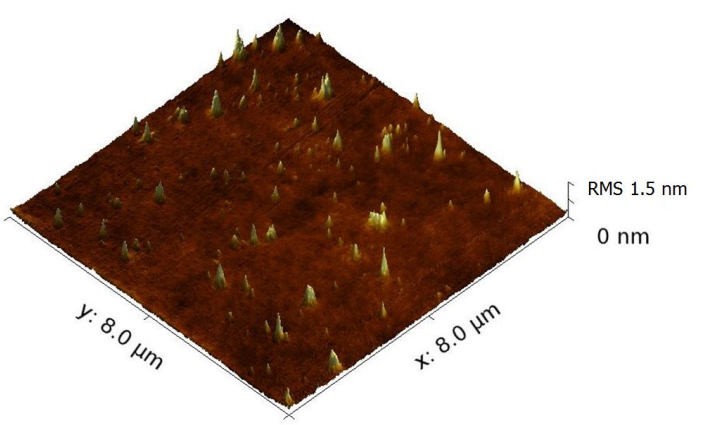

(b)

Figure 3.14: AFM of interface layers: a)Fabink-UV-IF4 b)CM116-20

The thickness of textile with interface layers was measured using a caliper and equals approximately $0.9 \mathrm{~mm}$ for both CM116-20 and Fabink-UF-IF4. The thickness of interface layers was estimated using Scanning Electro Microscopy (SEM) shown in Fig 3.13. Since the same stencil was used for the formation of both interface layers, their resulting thickness is approximately identical and equals to $100-350 \mu \mathrm{m}$. This considerable variation stems from the warp and weft yarns that, when intersect, result in repeating hills and deeps. In the hills region, the interface layer thickness decreases up to $100 \mu \mathrm{m}$, whereas in deeps the thickness increases up to $350 \mu \mathrm{m}$.

Atomic Force Microscopy that is shown on Fig 3.14 was used to measure the surface roughness of both interface layers. Fabink-UV-IF4 interface layer exhibited RMS surface roughness of 10nm whereas CM116-20 demonstrated RMS surface roughness of $1.5 \mathrm{~nm}$. Given that the overall thickness of a printed conductive layer is more than $500 \mathrm{~nm}$, these surface roughness values are satisfying. Nevertheless, according to AFM measurements in these samples the CM116-20 interface layer is about six times smoother than the surface of the Fabink-UV-IF4 interface layer. The RMS surface roughness values are very small and can be caused by dust or other pollutants that attach to the surface.

Table 3.3 summarizes the properties of interface layers constructed by Fabink-UVIF4 and CM116-20. 
Table 3.3: Fabink-UV-IF4 and CM116-20 Comparison

\begin{tabular}{|c|c|c|}
\hline Property & Fabink-UV-IF4 & CM116-20 \\
\hline Rheology & polymer & polymer \\
\hline Color & clear transparent & clear yellowish \\
\hline Surface & uniform and flat & uniform and flat \\
\hline $\begin{array}{c}\text { Thickness } \\
\text { with fabric }\end{array}$ & $0.9 \mathrm{~mm}$ & $0.9 \mathrm{~mm}$ \\
\hline $\begin{array}{c}\text { Thickness of } \\
\text { interface layer }\end{array}$ & $100-350 \mu \mathrm{m}$ & $100-350 \mu \mathrm{m}$ \\
\hline $\begin{array}{c}\text { RMS } \\
\text { Surface Roughness }\end{array}$ & $10 \mathrm{~nm}$ & $1.5 \mathrm{~nm}$ \\
\hline $\begin{array}{c}\text { Curing time } \\
\text { Cost per gram }\end{array}$ & $2 \mathrm{~min}$ & 2 hours \\
\hline
\end{tabular}

It can be depicted from Table 3.3 that physical properties of interface layers produced by two inks - special interface paste Fabink-UV-IF4 and general purpose CM116-20 are very much alike. The CM116-20 can boast lower surface roughness whereas Fabink is more transparent. However, main differences are reflected in processing time and cost. Fabink-UV-IF4 possesses much less processing time while cost is considerably higher. CM116-20 is a cheap alternative, but curing time for this recipe is more than two hours. However, this recipe is not exclusive and with more research another recipe can be put together that will boast a shorter processing time. It is worth noting that purchasing costs can be decreased if ink is purchased in bulk quantities. Also, employing parallel manufacturing can effectively neutralize the long curing time requirement. For prototype manufacturing, CM116-20 is the better choice since it is cheaper, so it can be used more freely. Therefore, in this research work, the conductive printing of the complete circuit layout of the localization device will be conducted on the CM116-20 interface layer. 


\subsection{Characterization}

Since the dielectric paste (CM116-20) has been selected and an interface layer is successfully formed on top of a textile substrate, a conductive printing process can take place. The conductive printing is accomplished using Dimatix 2831 ink-jet printer with the ANP DGP 40LT-15C silver nano-particle ink with 20um drop spacing. The sample deposited circuit layout(before thermal sintering) is represented in Fig 3.15 .

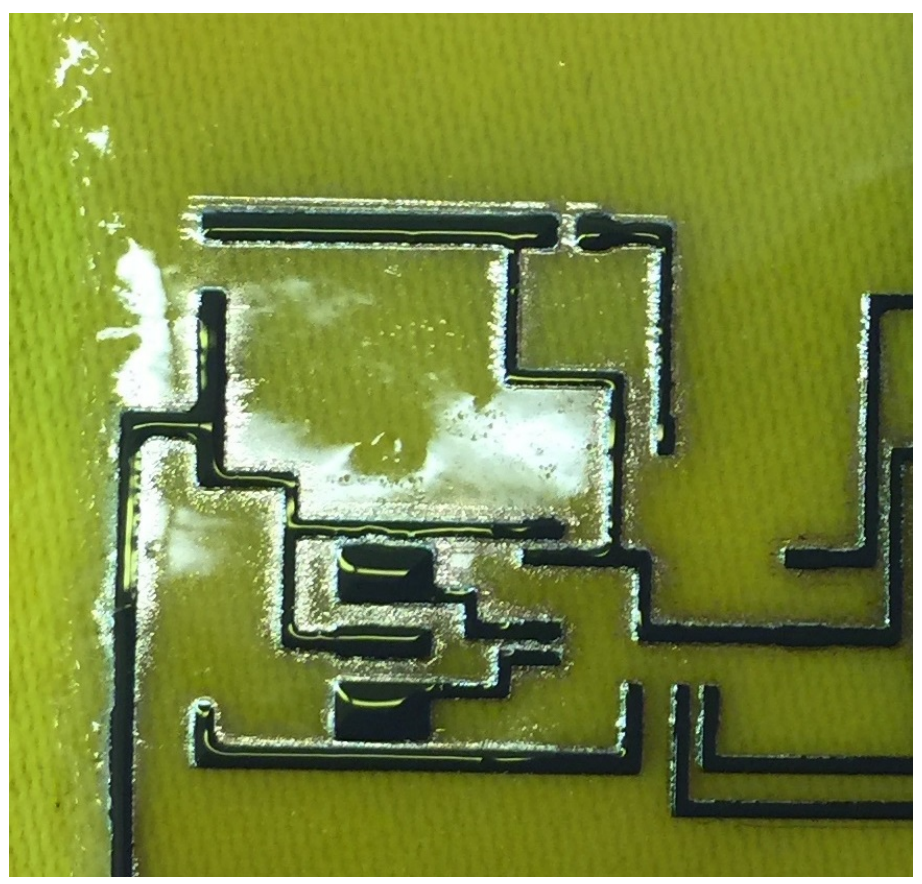

Figure 3.15: Deposited conductive layout of the localization device.

In order to print the final circuit layout, the extensive characterization of conductive printing and interface layer properties on the CM116-20 interface layer was accomplished. In this section, different parameters characterization on three textiles - 85\%/15\% polyester/cotton (Fig 3.11), 65\%/35\% polyester/cotton (Fig 3.16(a) and $100 \%$ cotton (Fig 3.16(b) will be studied. 


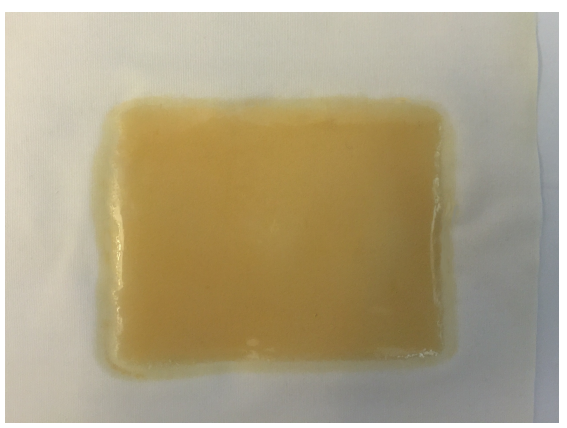

(a)

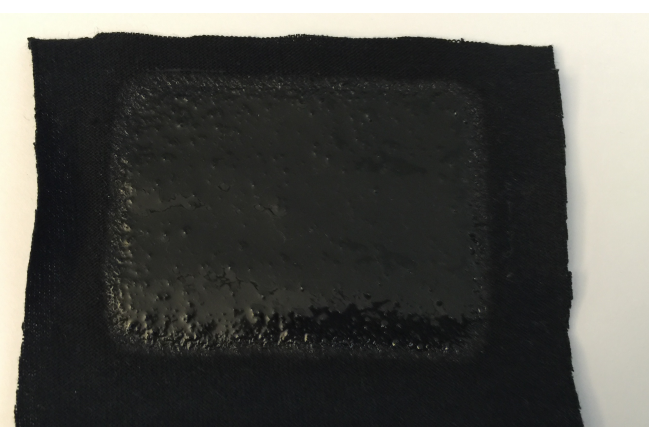

(b)

Figure 3.16: CM116-20 interface layer on: a) 65\%/35\% polyester/cotton b) $100 \%$ cotton

It is worth noting that according to Fig 3.16 the interface layer formation procedure using CM116-20 and 0.3mm thick PET stencil produces two visually different interface layers. The interface layer on $65 \% / 35 \%$ polyester/cotton shown on Fig.3.16(a) looks thick and smooth, however on Fig $3.16(\mathrm{~b})$ the interface layer on $100 \%$ cotton is considerably coarse. Distinct hillocks can be seen all across the $100 \%$ cotton interface layer.

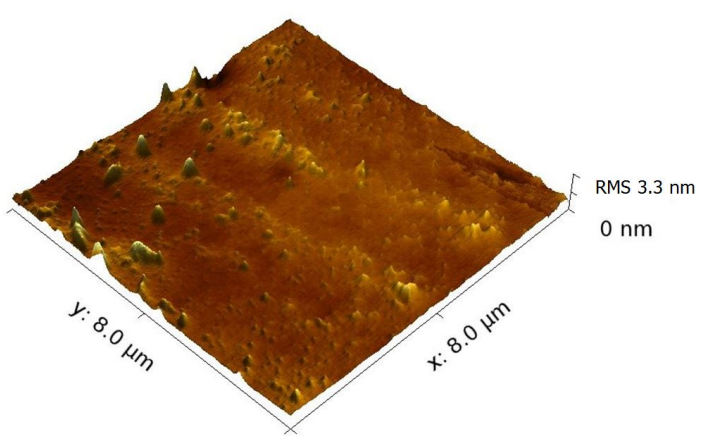

(a)

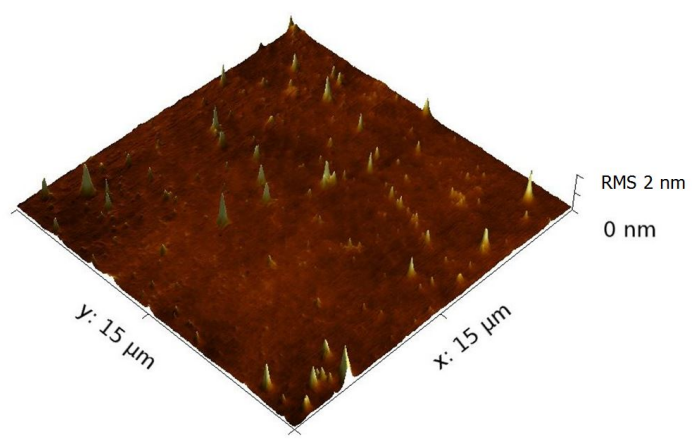

(b)

Figure 3.17: AFM of interface layer on: a) 65\%/35\% polyester/cotton b) $100 \%$ cotton

However, the perceived difference in surface roughness is not supported by AFM images of these two interface layers shown on Fig 3.17. The RMS surface roughness of $65 / 35$ polyester/cotton is $3.3 \mathrm{~nm}$ whereas it is equal to $2 \mathrm{~nm}$ for $100 \%$ cotton. These 
results are almost same. Such occurrence can be explained by the fact that in reality hillocks are separated by valleys of much flatter surface similar to the surface of $65 \% / 35 \%$ polyester/cotton interface layer. The existence of hillocks stems from the interface layer thickness produced by $0.3 \mathrm{~mm}$ stencil. For $65 / 35$ polyester/cotton substrate, which is thin and relatively smooth, such interface layer thickness is clearly redundant, and flat surface can be achieved using a thinner stencil. However, for $100 \%$ cotton substrate that is very rough and has high porosity, the $0.3 \mathrm{~mm}$ stencil thickness is not enough to cover all innate roughness, leaving some hillocks uncovered. These hillocks are detrimental to the printed conductive layers. Nevertheless, in this research work, only interface layers created by $0.3 \mathrm{~mm}$ PET stencil are studied.

Both $100 \%$ cotton and $65 \% / 35 \%$ polyester/cotton are bendable enough as it can be seen on Fig 3.18 .

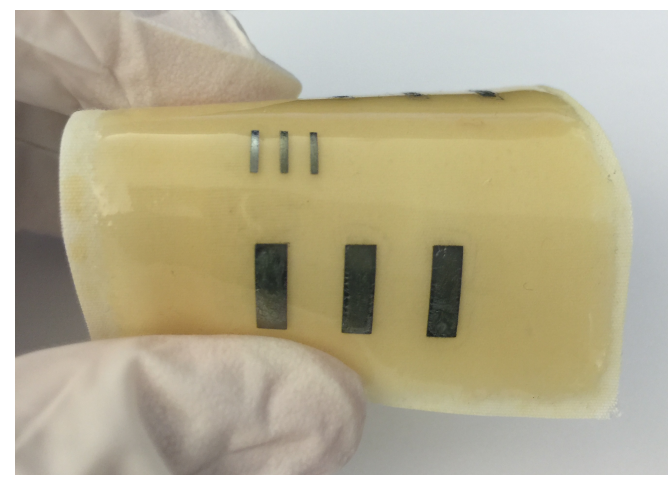

(a)

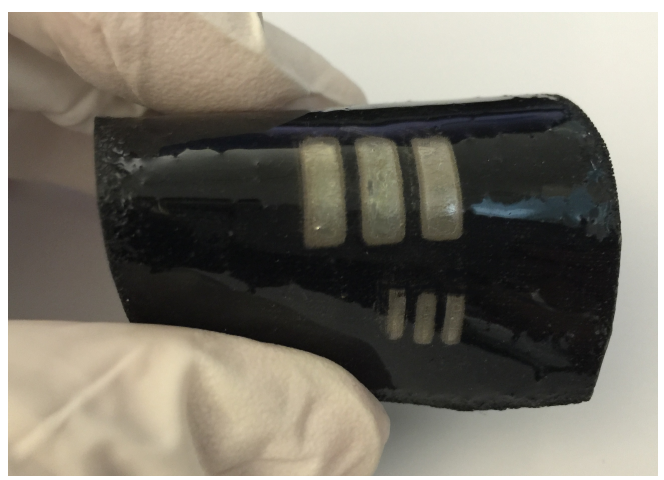

(b)

Figure 3.18: Demonstrating flexibility of: a) 65\%/35\% polyester/cotton b) $100 \%$ cotton

\subsubsection{Electrical Properties of Conductively Printed Layers on Interface Layer}

Electrical properties studied in this section include sheet resistance, volume resistivity and conductivity. Sheet resistance $R_{s h}(\Omega / s q$.$) is derived using four-point probe$ 
method and 4.2357 correction factor. Volume resistivity $\rho(\Omega \cdot m)$ and conductivity $\sigma(S / m)$ can be calculated from sheet resistance using Eq.3.1 and Eq 3.2 respectively.

$$
\rho=R_{s h} \cdot t
$$

Where $t$ is a thickness of a conductive layer in $\mathrm{m}$.

$$
\sigma=\frac{1}{\rho}
$$

To study the electrical properties of conductive printing on the interface layer the structure presented on Fig. 3.19 was printed. Eight lines $(5 \times 1 \mathrm{~mm})$ and eight rectangles $(10 x 3 m m)$ were printed on the CM116-20 interface layer formed on 85\%/15\% polyester/cotton substrate. Printing on other textile materials will be discussed later. Lines and rectangles are printed using the different number of conductive layers. Layers grow from two to nine. The 20 min thermal sintering at $130^{\circ} \mathrm{C}$ was performed after the printing of each two layers. Every rectangle is paired with the line that was constructed using the corresponding number of layers(i.e. the two layers line corresponds to the two layers rectangles and so on). Rectangles are used in four-point probe sheet resistance measurements and lines are required to calculate the approximate layers thickness using profilometer. Data derived from lines and rectangles is combined to produce resistivity and conductivity values. It can be observed that the rectangle surfaces are noticeable fissured. However, from Table 3.4 it seems that conductivity is not affected much by these fissures. 


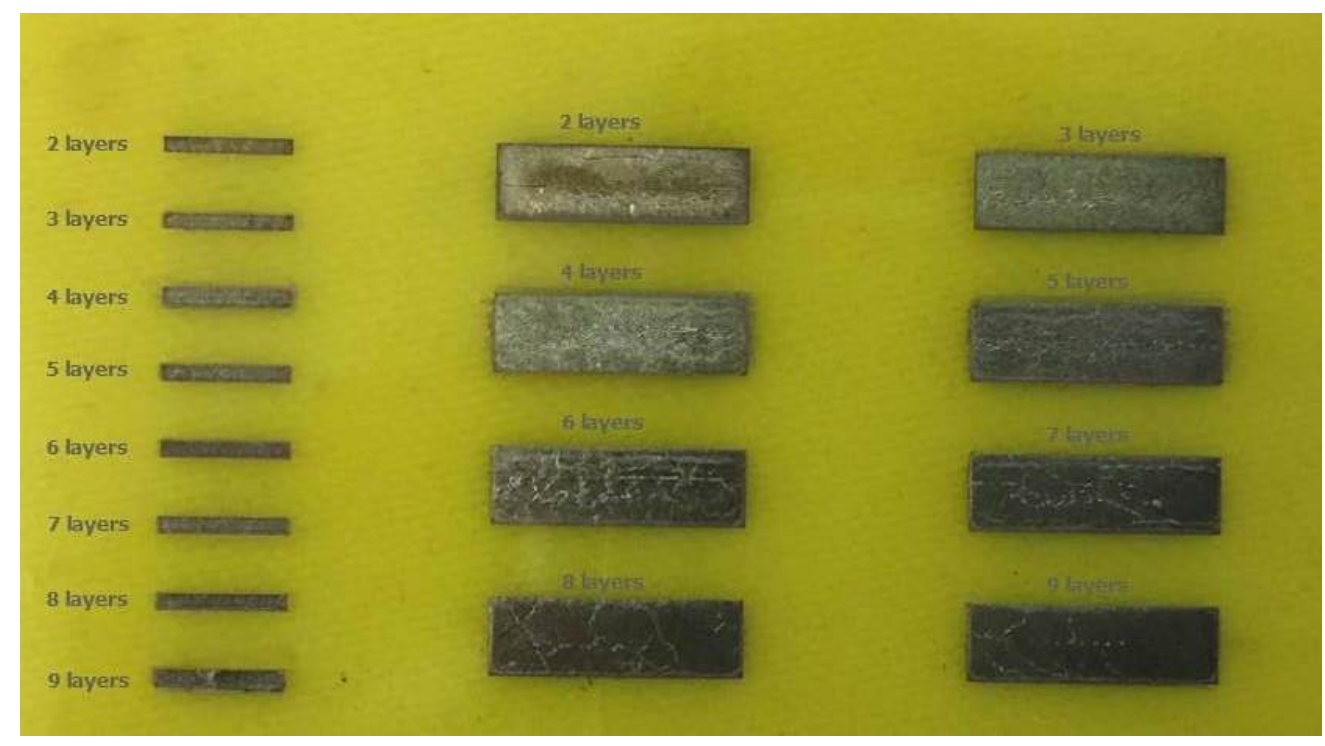

Figure 3.19: 8 lines and 8 rectangles for electric properties measurements.

Table 3.4 summarizes measured and calculated height, sheet resistance, bulk resistivity and conductivity of various number of printed layers.

Table 3.4: Electrical properties of conductive layers on $85 \% / 15 \%$ polyester/cotton.

\begin{tabular}{|l|c|c|c|c|c|c|c|c|}
\hline \# of layers & $\mathbf{2}$ & $\mathbf{3}$ & $\mathbf{4}$ & $\mathbf{5}$ & $\mathbf{6}$ & $\mathbf{7}$ & $\mathbf{8}$ & $\mathbf{9}$ \\
\hline thickness $(\mu \mathrm{m})$ & 5 & 6 & 7 & 8 & 9 & 11 & 12 & 13.5 \\
\hline sheet resistance $R_{s h}(m \Omega / s q)$. & $*$ & 1600 & 370 & 135 & 60 & 55 & 40 & 34 \\
\hline resistivity $\rho(\mu \Omega \cdot m)$ & $*$ & 9.6 & 2.59 & 1.08 & 0.54 & 0.6 & 0.42 & 0.46 \\
\hline conductivity $\sigma(M S / m)$ & $*$ & 0.1 & 0.39 & 0.926 & $\mathbf{1 . 8 5}$ & 1.92 & 2.08 & 2.15 \\
\hline
\end{tabular}

It can be seen from the Table 3.4 that the values for two conductive layers are starred. This does not mean that two printed layers are not conductive. It implies that two layers have a very high sheet resistance that is out of the CMT-SR2000N four point probe system's measurement range that, according to the data sheet, equals to $1 m \Omega / s q .-2 M \Omega / s q$. . The presence of conductive was proven by the multimeter DC resistance measurement that showed the value of $6 M \Omega$. Thus, at least three printed layers and two thermal sintering cycles of 20 mins at $150^{\circ} \mathrm{C}$ each are needed. The conductivity starts to increase with the number of layers. Fig 3.20 shows that the conductivity is steadily improving; however, the highest increase occurs when six 
conductive layers are printed. After six layers, conductivity improvements are not as impressive. Therefore, six conductive layers formed by three printing and sintering cycles are optimal for the formation of printed structures on CM116-20 interface layer with $85 \% / 15 \%$ polyester/cotton textile substrate. The conductivity of $1.85 \mathrm{MS} / \mathrm{m}$ exhibited by six ink-jet printed layers is an order of magnitude lower than the conductivity of typical "electronic" metals such as silver $(63 \mathrm{MS} / \mathrm{m})$ and $\operatorname{copper}(59.6 \mathrm{MS} / \mathrm{m})$. Nevertheless, such conductivity is still adequate for circuit layout printing, and it only affects the minimum circuit resolution i.e. the minimum usable trace width. Since the printed conductivity is not on par with the conductivity of copper, it will not be possible to achieve the trace resolution similar to traditional PCB production. Achievable trace resolution will be investigated in Section 3.3.2.

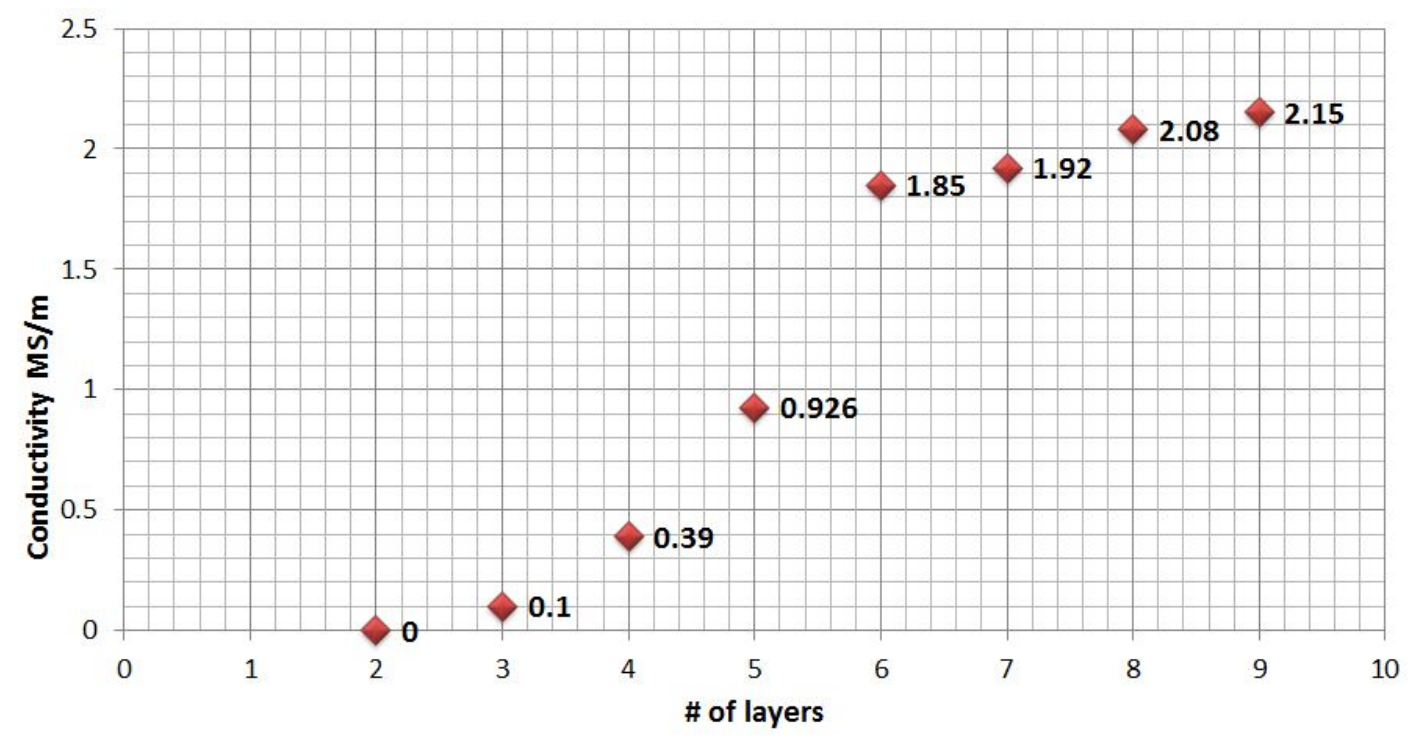

Figure 3.20: Conductivity dependency on the number of layers.

Electrical properties of printed conductive layers on $100 \%$ cotton and $65 \% / 35 \%$ polyester/cotton textile substrates are analyzed in the same manner. However, for these two substrates lesser number of layers were considered - only lines and rectangles of four, six and eight layers were fabricated via two, three and four printing and sintering cycles respectively. Table 3.5 summarizes electrical properties of conductive 
layers printed on the CM116-20 interface layer formed on $100 \%$ cotton and $65 \% / 35 \%$ polyester/cotton textile substrates. It can be spotted that $65 \% / 35 \%$ polyester/cotton exhibits very similar conductivity to $85 \% / 15 \%$ polyester/cotton analyzed earlier. Such behavior was expected because maximum surface roughness values are very close. However, the conductivity of four and six layers printed on $100 \%$ cotton is much lower when compared to the $85 \% / 15 \%$ polyester/cotton. Lower conductivity stems from the insufficient thickness of the interface layer that was not enough to completely cover the weaving of $100 \%$ cotton and resulted in the formation of hillocks that impede achieving same conductivity values. Consequently, the thicker interface layer will lead to improved conductivity that will allow successful printing on $100 \%$ cotton, however, the overall flexibility might decrease. Also, as it can be seen from Table 3.5, the eight conductive layers printed on $100 \%$ cotton produce comparable conductivity to $65 \% / 35 \%$ polyester/cotton.

Table 3.5: Electrical properties of conductive layers on $65 \% / 35 \%$ polyester/cotton and $100 \%$ cotton substrates

\begin{tabular}{|l|c|c|c|c|c|c|}
\hline & \multicolumn{3}{|c|}{$\mathbf{1 0 0 \%}$ cotton } & \multicolumn{3}{c|}{$\mathbf{6 5 \%} / \mathbf{3 5 \%}$ poly/cotton } \\
\hline \#of layers & $\mathbf{4}$ & $\mathbf{6}$ & $\mathbf{8}$ & $\mathbf{4}$ & $\mathbf{6}$ & $\mathbf{8}$ \\
\hline thickness $(\mu \mathrm{m})$ & 7 & 9 & 12 & 7 & 9 & 12 \\
\hline sheet resistance $R_{s h}(m \Omega / s q)$. & 5332 & 610 & 58 & 262 & 70 & 47 \\
\hline resistivity $\rho(\mu \Omega \cdot m)$ & 37.3 & 5.49 & 0.7 & 1.83 & 0.63 & 0.56 \\
\hline conductivity $\sigma(M S / m)$ & 0.027 & 0.18 & 1.43 & 0.55 & 1.59 & 1.78 \\
\hline
\end{tabular}

\subsubsection{Conductive Trace Resolution}

Since the sufficient conductivity can be achieved by printing six conductive layers on the interface layer, the next step is to determine what minimum trace width can be accomplished by ink-jet printing. The minimum trace width is estimated using the simple arrangement shown in Fig.3.21. 


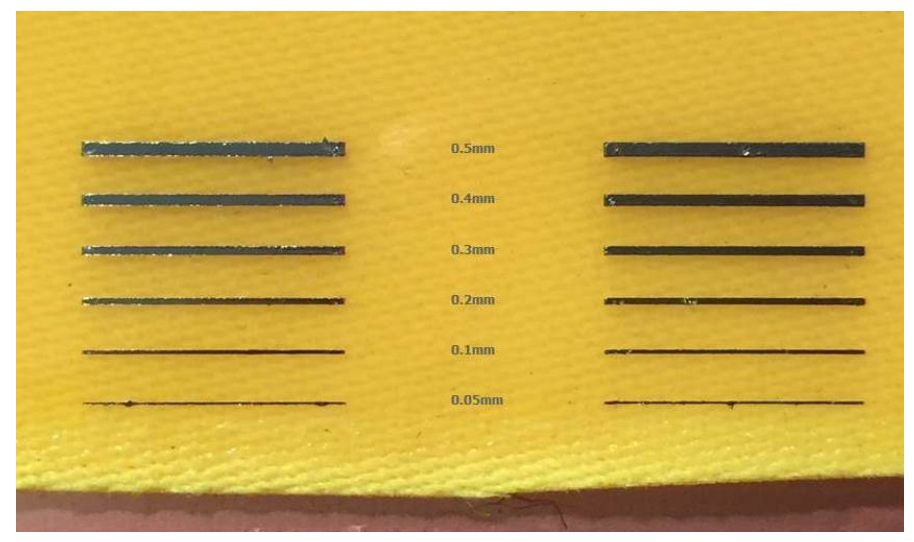

Figure 3.21: Minimum trace width measurement.

Two samples of six lines each were printed using three printing and sintering cycles resulting in six conductive layers. All six traces are of $10 \mathrm{~mm}$ length but of different width $-0.5,0.4,0.3,0.2,0.1$ and $0.05 \mathrm{~mm}$. The simple DC resistance measurement was performed by placing multimeter tips on line edges. Resulting DC resistance is represented in Table 3.6.

Table 3.6: DC resistance of traces with different width

\begin{tabular}{|l|c|c|c|c|c|c|}
\hline Trace width $(\mathrm{mm})$ & 0.5 & 0.4 & 0.3 & 0.2 & 0.1 & 0.05 \\
\hline DC resistance $(\Omega)$ left & 0.6 & 0.73 & 0.97 & 1.5 & $*$ & $*$ \\
\hline DC resistance $(\Omega)$ right & 0.55 & 0.74 & 1.04 & 1.4 & $*$ & $*$ \\
\hline
\end{tabular}

Traces up to $0.2 \mathrm{~mm}$ in width exhibited some DC resistance values. No successful DC resistance measurements of $0.1 \mathrm{~mm}$ and $0.05 \mathrm{~mm}$ traces were achieved, even though ink-jet printer can reliable print conductive lines up to 50 micron. The possible explanations of unsuccessful readings include very high resistivity, excessive surface roughness or open circuits along the lines. If thin lines up to $0.05 \mathrm{~mm}$ need to be printed, the printing should be optimized further with more investigation done. The bottom line is that for this research's localization circuit printing purposes 0.1 and $0.05 \mathrm{~mm}$ trace widths are not going to be used. Therefore, traces up to $0.2 \mathrm{~mm}$ in width will be used as a minimum circuit feature size, however, due to considerable DC resistance, structures featuring width of $0.2 \mathrm{~mm}$ should be kept relatively short. 
65

\subsubsection{RF Properties of Interface Layer}

Electromagnetic properties of materials such as relative permittivity $\left(\varepsilon_{r}\right)$ and $\operatorname{loss}$ $\operatorname{tangent}(\tan \delta)$ are critical in the design of RF structures such as antennas. Since an antenna is an integral part of any tracking device, the permittivity and loss tangent of interface layers on textile substrates should be acquired. These parameters are measured using the Agilent E4991A Material Analyzer. Since the formed interface is chemically linked to the textile substrate, the separate analyses of the interface and textile layers are not possible. Therefore, the whole interface and textile stack up was analyzed simultaneously. The Fig 3.22 presents the measurement of $\varepsilon_{r}$ (Fig.3.22(a) and $\tan \delta($ Fig, $3.22(\mathrm{~b})$ of the CM116-20 interface layer and 85\%/15\% polyester/cotton stack up.

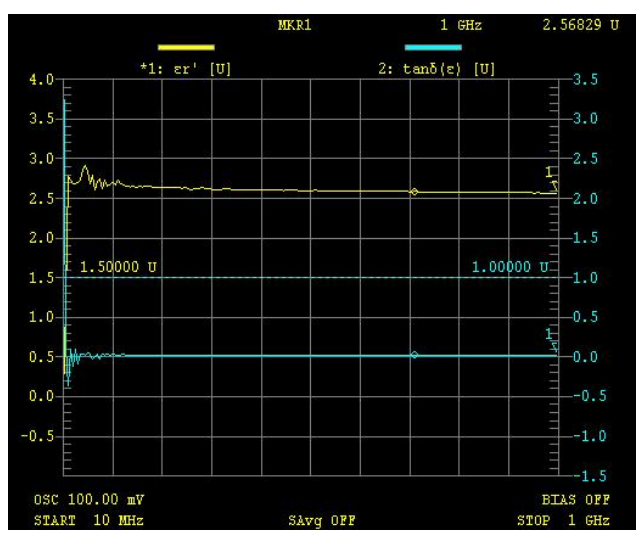

(a)

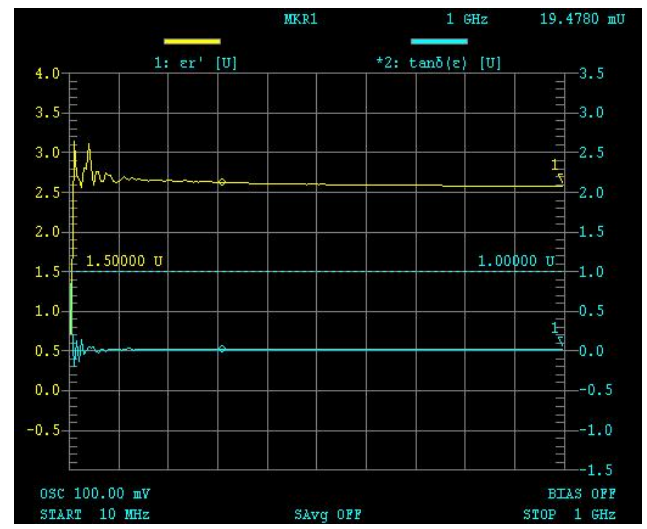

(b)

Figure 3.22: Measurement of: a) permittivity $\left(\varepsilon_{r}\right)$ b) loss $\operatorname{tangent}(\tan \delta)$

Table 3.7 summarizes RF properties for different textile materials. The values for permittivity and loss tangent are close, and the value variation can be attributed to the difference in textile substrates. The measured values for $85 \% / 15 \%$ polyester/cotton will be used for the antenna optimization in Chapter 4. 
Table 3.7: Permittivity and loss tangent for three textile substrates

\begin{tabular}{|l|c|c|c|}
\hline & $\mathbf{8 5 \%} / \mathbf{1 5 \%} \mathbf{~ p o l y} / \mathbf{c o t t o n}$ & $\mathbf{6 5 \%} / \mathbf{3 5 \%} \mathbf{~ p o l y} /$ cotton & $\mathbf{1 0 0 \%}$ cotton \\
\hline permittivity $\left(\varepsilon_{r}\right)$ & 2.57 & 2.87 & 2.93 \\
\hline loss tangent $(\tan \delta)$ & 0.019 & 0.019 & 0.028 \\
\hline
\end{tabular}

\subsubsection{Bending analysis}

The ability to remain conductive while being constantly subjected to bending is paramount for Smart Fabrics. Ordinary clothes bend, curve and twist all the time. Interface layers formed on textiles are flexible, thus, they also will be exposed to repetitive bending. It is practical to measure whether conductive lines printed on interface layers can withstand several bending cycles. To quantify the conductivity degradation, fifty bending cycles of the rectangle(10x5mm), printed on CM116-20 interface layer formed on $85 \% / 15 \%$ polyester/cotton textile substrate will be performed as shown on Fig 3.23 . The rectangle consists of six conductive layers constructed using three printed and sintering cycles. DC resistance will be measured after each five bending cycles by placing multimeter tips on rectangle edges.

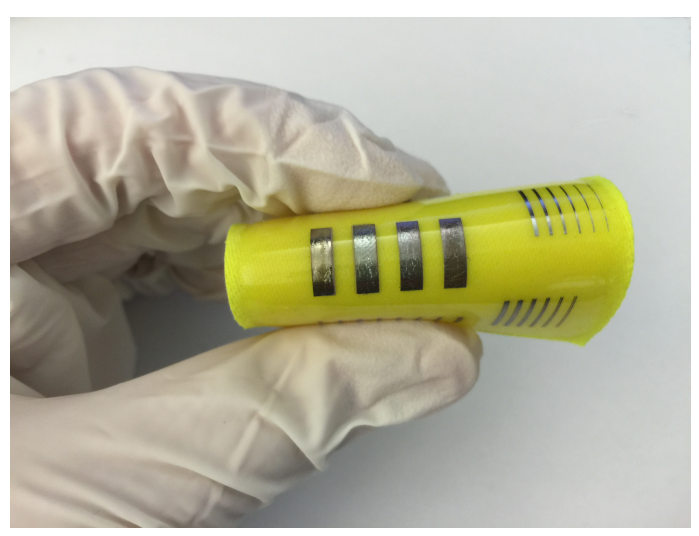

(a)

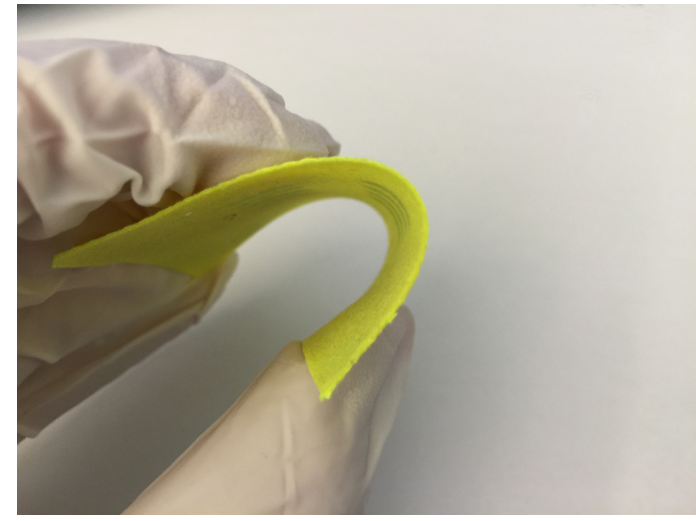

(b)

Figure 3.23: Interface layer bending to check structure conductivity a) front view b) side view

Table 3.8 summarizes the change in DC resistance with the number of bending 
cycles outlined on Fig 3.23 . It can be seen that the DC resistance does not considerably change even after fifty bending cycles. This fact suggests that a circuit layout printed on an interface layer will be able to withstand at least fifty severe physical deformations.

Table 3.8: Bending Impact on DC resistance

\begin{tabular}{|c|c|c|c|c|c|c|c|c|c|c|c|}
\hline \# bending cycles & 0 & 5 & 10 & 15 & 20 & 25 & 30 & 35 & 40 & 45 & 50 \\
\hline DC resistance $(\Omega)$ & 0.32 & 0.33 & 0.34 & 0.35 & 0.32 & 0.33 & 0.37 & 0.34 & 0.39 & 0.35 & 0.34 \\
\hline
\end{tabular}

\subsection{Summary}

The previous chapter established the Interface Layer method as a very promising for E-textiles industry because the method excels in the printed circuit production similar to traditional PCB manufacturing while preserving relative textiles flexibility. In this chapter, conductive printing via Interface Layer method was studied in more details. First, interface layer fabrication requirements were discussed, and specific technical directions were given. Next, interface layer formation steps using two dielectric inks - the specifically designed interface paste Fabink-UV-IF4 and the general purpose dielectric coating CM116-20 were derived. Resulting interface layers created with two different dielectric inks were compared. It turned out that physically interface layers are very similar - clear, flat, polymer surfaces with relatively low surface roughness. However, the fabrication of interface layer from the Fabink-UV-IF4 ink is faster but the material is expensive, whereas the CM116-20 paste provides a slower but lower cost alternative. The final section was devoted to the characterization of interface layers and conductive structures printed on them. It was measured that six conductive layers printed on the CM116-20 interface layer provide adequate conductivity, trace resolution up to $0.2 \mathrm{~mm}$ and remain conductive even after continuous bending. Using six layers and derived minimum feature width, any structure, including circuit layouts, can be conductively printed on textile via the Interface Layer method. 
After being able to fabricate the interface layer using general purpose dielectric ink and acquiring satisfactory characterization results, the only logical step is to print the complete localization circuit layout on fabric and build the final device. This steps along with antenna optimization and field tests will be discussed in the next chapter. 


\section{Chapter 4}

\section{Localization Device: Design, Fabrication, and Testing}

In the previous chapter, the interface layer formation process utilizing the general purpose CM116-20 dielectric ink was derived. Next, conductive printing was performed, and key parameters were analyzed. Thus, all required tools - the interface layer process and primary specification of conductive printing on the interface layer, are in place for the ultimate fabrication of a tracking device on fabric.

In this chapter, the WiFi tracking device printed directly on textile using the Interface Layer method will be created. Its prototyping procedures using standard PCB techniques will be discussed. After that, on-textile prototype fabrication steps, its characterization and field tests results will be shown. Finally, the completed tracking device incorporated on T-shirt will be presented.

\subsection{Prototyping}

Every device production cycle starts from the prototyping. Prototyping is the creation of a sample model that will be used to test a concept i.e. localization. The initial localization prototype will be manufactured using a regular printed circuit board manufacturing. The PCB prototype will be used to prove that hardware, firmware 
and software act properly. Once it is successful, the prototype will be modified to function on the textile substrate. All prototyping is carried out in-house, KAUST IMPACT Lab.

\subsubsection{System Design}

The abstract system design is always the first step in the prototype creation. System design provides a general overview of the intended device operation. System design for the localization device is presented in Fig 4.1 .

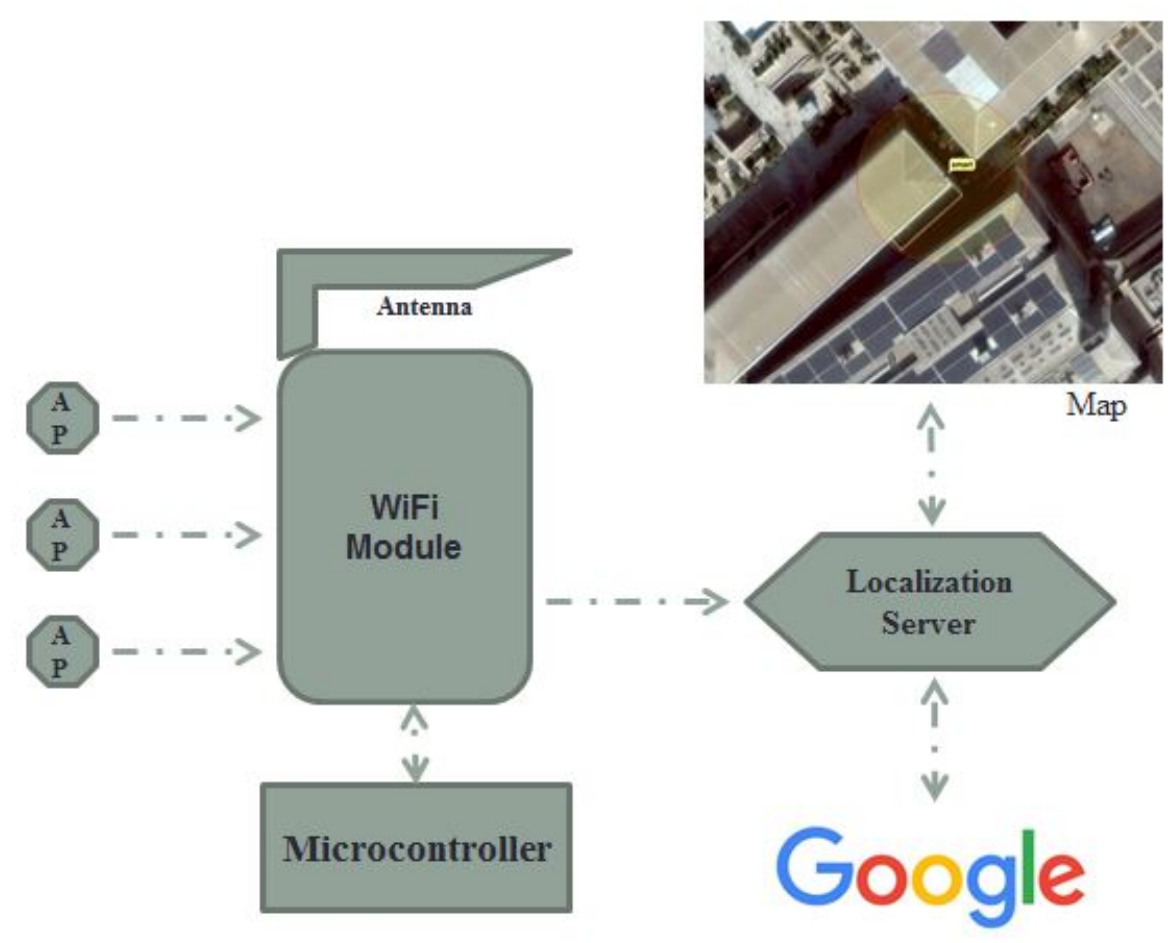

Figure 4.1: System design of the localization device

In short, the localization device scans nearby access points, formats this data, and sends it to a server where the access points data is used by the Google Geolocation API to locate the position of the localization device on a map. In more details, the WiFi access points such as WiFi routers allow different devices to connect to 
WiFi network. WiFi access points (or nodes) can broadcast different WiFi networks. Each WiFi node has its unique identifier - MAC address. API stands for Application Program Interface and is used to assist developers in writing code that interfaces with other software. In this research, Google Geolocation API is used. This API provides the interface to embed Google's Geolocation database and its triangulation algorithms into the code. In more details, the system design can be explained as follows:

- A WiFi module that is presented in the center of Fig.4.1 is the major part of the localization device. The WiFi module provides wireless LAN access. Such module has an on-board TCP/IP stack, it can automatically join a WiFi network, and send and/or receive data packets.

- The WiFi module is mainly used to scan nearby WiFi access points that are shown on the left on Fig 4.1 .

- Access points data that is scanned by WiFi module is then transported to a microcontroller. The microcontroller is the brain of this localization circuit. It processes the nodes data, formats it and sends the requests with access points data to the localization server.

- An antenna was deliberately emphasized because it is a very crucial part of the localization system. The antenna is used for reading access points, connecting to the WiFi network, and communicating with a server. Since the antenna will be conductively printed on the textile, the particular attention should be given to its proper functioning.

- The server is created to host specifically the localization application. The server's internal application reads the received nodes data, formats it and pumps it through the Google API to receive the location coordinates and accuracy radius. Then, the server application plots these values on a localization map. 
- The Google Maps Geolocation API is the service that is universally used for the WiFi-based localization. It takes WiFi nodes data, processes it through the vast WiFi access points database and then uses proprietary algorithms to return three values - latitude, longitude, and accuracy. Latitude and longitude points are used to pin the estimated location (center of the yellow circle on the map on Fig, 4.1), whereas accuracy specifies the localization search radius in meters (the complete yellow circle on the map on Fig,4.1).

- The map is used to provide an interface needed to visualize the location of the device. The map used in this research is the custom version of Google Maps. This map can be accessed from any Internet-enabled device such as laptops, mobile phones, etc., thus allowing for position tracking from any place where Internet is present.

The system level design is then implemented in hardware, firmware and software of the PCB prototype.

\subsubsection{Prototype Hardware}

In order to quickly and efficiently debug and tweak prototype's firmware and software, the localization prototype's hardware should be kept relatively simple but at the same time functional. The prototype's schematic is presented on Fig.4.2. The schematic is constructed via Eagle CAD software. 


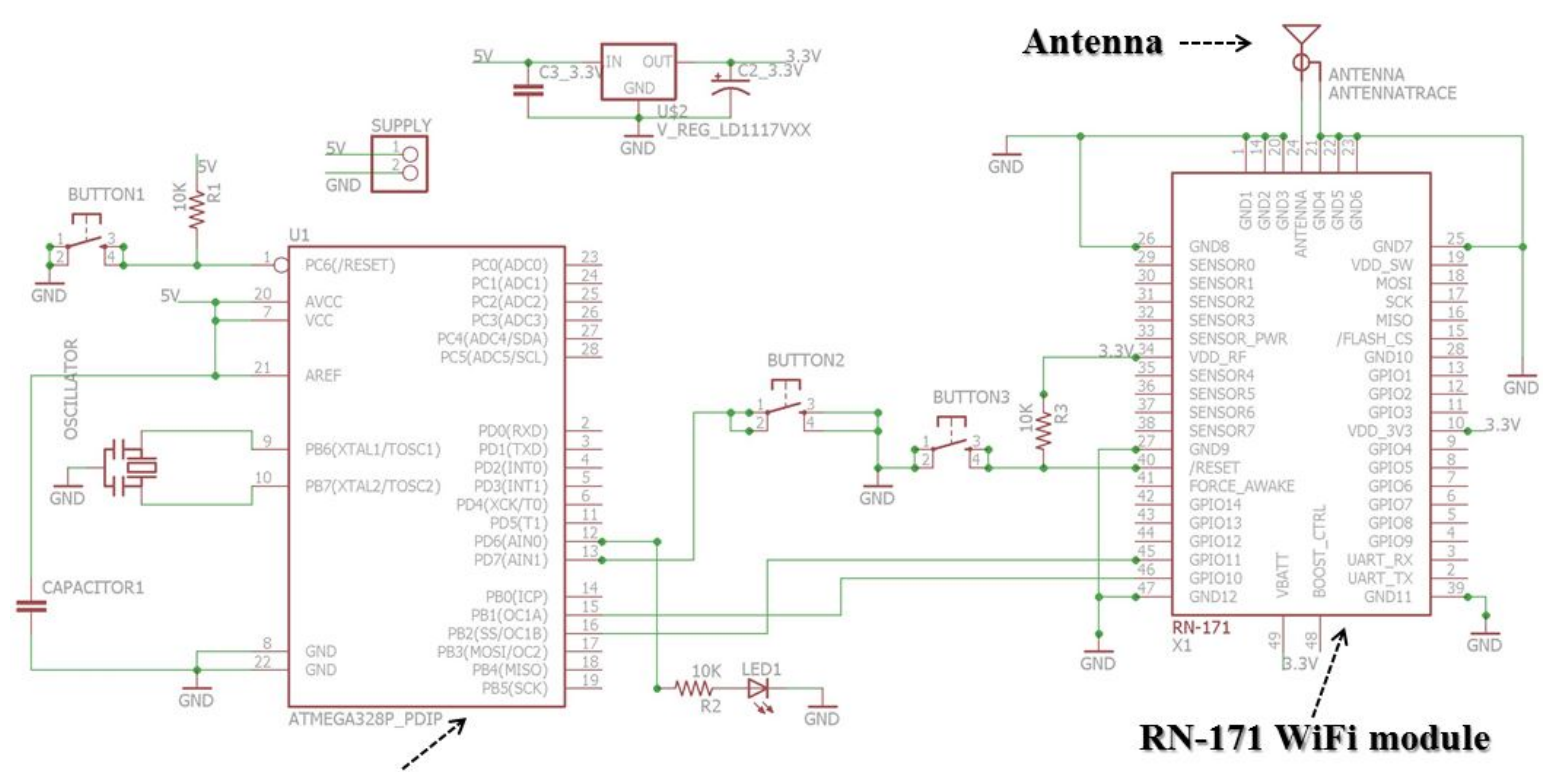

Microcontroller

Figure 4.2: Localization prototype schematic

It can be seen from the Fig 4.2 that the localization prototype's hardware is not particularly difficult. It consists of the following components:

- RN171 WiFi module that is responsible for the wireless connection. This particular module was selected due to its employment simplicity and that it does not require a lot of additional components to function properly.

- Atmega328P that is a very popular and simple AVR 8-bit microcontroller. It is powerful enough to handle all processing requirements, has a sufficient number of IO lines, and supports serial communication interfaces.

- Antenna is a very critical component for any localization device. For the prototyping purposes, the inverted F-type antenna is used. This antenna is optimized for the textile substrate in section 4.2.1.

- All other discrete components such as resistors, capacitors, voltage dividers, LED and buttons are needed to ensure the proper operation of the WiFi mod- 
ule and the microcontroller by supplying appropriate voltage, status checking, providing resetting capabilities, etc.

The prototype circuit design is developed using EAGLE CAD and shown on Fig $4.3(\mathrm{a})$. This circuit layout is then referenced by LPKF milling machine to create the prototype PCB from a FR-4 substrate. Components are then soldered to produce the final PCB prototype presented on Fig.4.3(b). This prototype PCB is needed to test the operation of localization device's firmware and software. It can be seen that there is a socket instead of the Atmega328P microcontroller. This socket is needed to provide the circuit with fast microcontroller programming capabilities, thus no desoldering is required.

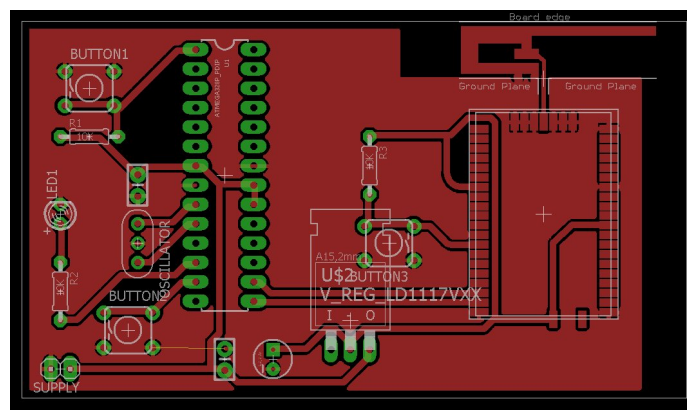

(a)

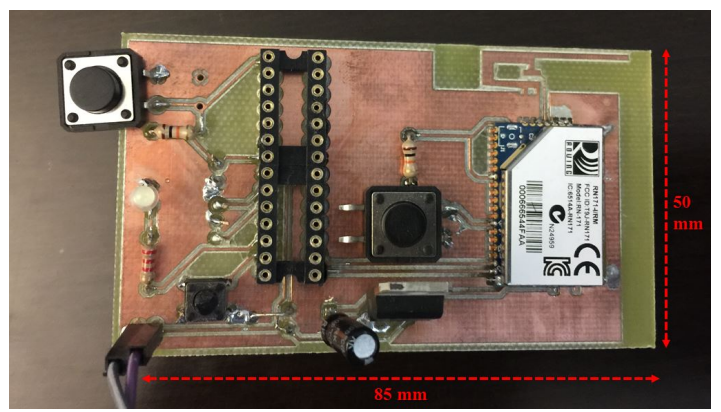

(b)

Figure 4.3: Localization prototype: a) circuit layout b) final PCB prototype

\subsubsection{Prototype Firmware and Software}

Firmware is the program that goes into the microcontroller, and software is the code that operates on the server side. The full code for both firmware and software is given in the Appendix A. In this section only code's governing principles are described.

Microcontroller code is written in $\mathrm{C}++$ using the Arduino development environment. Basically, the code operates the RN-171 module to scan ten nearby WiFi access points. RN-171 returns access point data stream in the following format: 


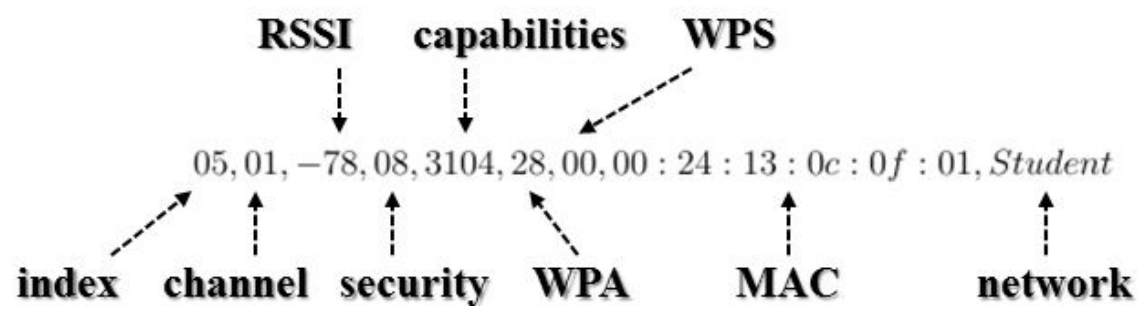

This data contains information regarding a single WiFi access point, however for localization purposes only channel, RSSI and MAC address parameters are required. RSSI stands for Received Signal Strength Indicator and measures WiFi network power level that a device is receiving from a WiFi node. The microcontroller's firmware extracts these values for each access point and then packs this data into one POST command that will be sent to the server via RN171 WiFi module. The firmware is also responsible for establishing serial data communication between RN171 and the microcontroller, as well as operating a status LED that signals in two cases - a) when the device is reading $\mathrm{WiFi}$ nodes, the LED produces a continual shimmering b) when the device is reset the LED flashes three times. LED is needed mainly for indication and debugging purposes and can be eliminated if necessary.

The localization server is operated via software written in PHP. Once the POST command is issued by the microcontroller, the server software is invoked. The software reads the data from the POST command, then formats it to send it to Google Geolocation API. The Google API processes the data comparing it to its node database and then sends the estimated longitude, latitude and accuracy back to the server. The server side software receives these three values and plots them on the customary map.

The prototype PCB that was created in the previous section is used to run and debug both firmware and software code. Once the code is properly checked, there is no need to modify it for the final on-textile localization device. 


\subsection{On-Textile Fabrication}

The fast prototype creation is a mature way to test and adjust the circuit design. In the previous section, the PCB prototype was created to test the circuitry, code and overall tracking operation. However, since the prototype was designed for PCB-based operation, some modifications are necessary to transfer the design onto the textile. Though the firmware and software do not need to be changed, the circuit itself needs to be modified in the following ways before the on-textile prototype can be created:

- The antenna should be optimized for textile substrates. Since the PCB antenna was specifically designed to radiate on a FR-4 substrate, changing the substrate will undoubtedly alter the antenna radiation parameters. Thus, the optimization and characterization of the antenna on textile is required.

- The prototype circuit should be simplified. Printing on textile is a more challenging process because of the minimum trace width. Inability to reliably produce very narrow conductive traces forces to use wider lines that restricts the number of interconnects that can be comfortably fitted into a given area. Thus, the number of components should be kept to a practical minimum. The good news is that the prototype circuit has a number of discrete components that are used solely for debugging. These components can be eliminated from the final on-textile circuit.

As it was discussed in previous chapters, the $85 \% / 15 \%$ polyester/cotton textile ordered from Klopman International will be used as a textile substrate for the localization circuit production. The CM116-20 dielectric ink from Creative Materials is used for the interface layer formation as discussed in Section 3.2.2. The conductive printing is accomplished via DGP 40LT-15C silver nano-particle ink from Advanced Nano Products. Each structure on the interface layer will consist of six printed conductive layers as it was discussed in Section 3.3.1. 


\subsubsection{On-Textile Antenna}

The modified inverted F-antenna (IFA) is used for both PCB and on-textile prototypes. This antenna is a $2.4 \mathrm{GHz}$ compact, low cost and high-performance antenna [66]. This antenna performed well with PCB prototype, however for textile substrate its parameters should be accordingly optimized. The modified inverted Fantenna that is used in prototyping is different from a standard IFA represented on

Fig 4.4. In the standard IFA configuration the radiating structure resembles inverted "F" letter. The standard IFA consists of a feed, right arm, left arm (shorting arm) and a large ground. Without the left arm, the resulting right arm and ground structure can be approximated as a dipole where the feed acts as a middle point. Such arrangement supplies capacitive impedance. In order to radiate, a left arm should be added. Depending on the length, the left arm provides a necessary inductive impedance that matches the capacitive impedance at a given frequency. As it can be seen from Fig 4.4, the standard IFA is different from the IFA used in prototyping. The shortening arm of the modified IFA is extended to provide adequate impedance matching without enlarging the antenna dimensions that is critical for maintaining the compactness of the final circuit. 


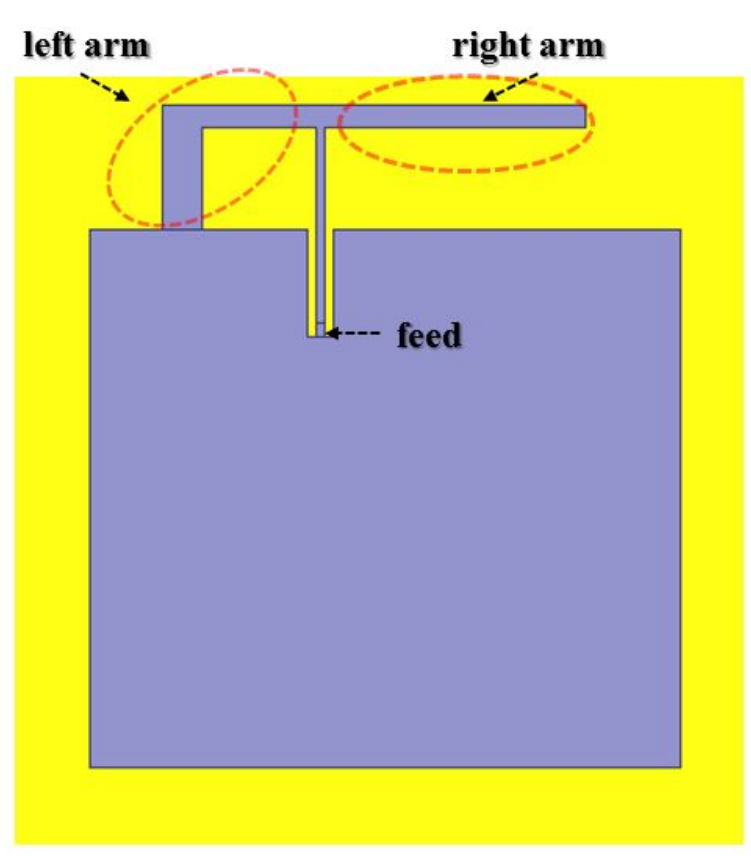

Figure 4.4: Standard inverted F-antenna simulated in HFSS

Utilizing the derived dielectric constant and loss tangent values for the interface layer on $85 \% / 15 \%$ polyester/cotton textile in conjunction with the measured conductivity of printed six silver ink layers, inverted F-antenna for the final on-textile circuit was customized in Ansys High Frequency Structure Simulator (HFSS). The simulated IFA model and corresponding parameters are given on Fig 4.5 and Table 4.1 respectively. This model and its simulation results will be used later to validate the actual antenna design printed on textile. 


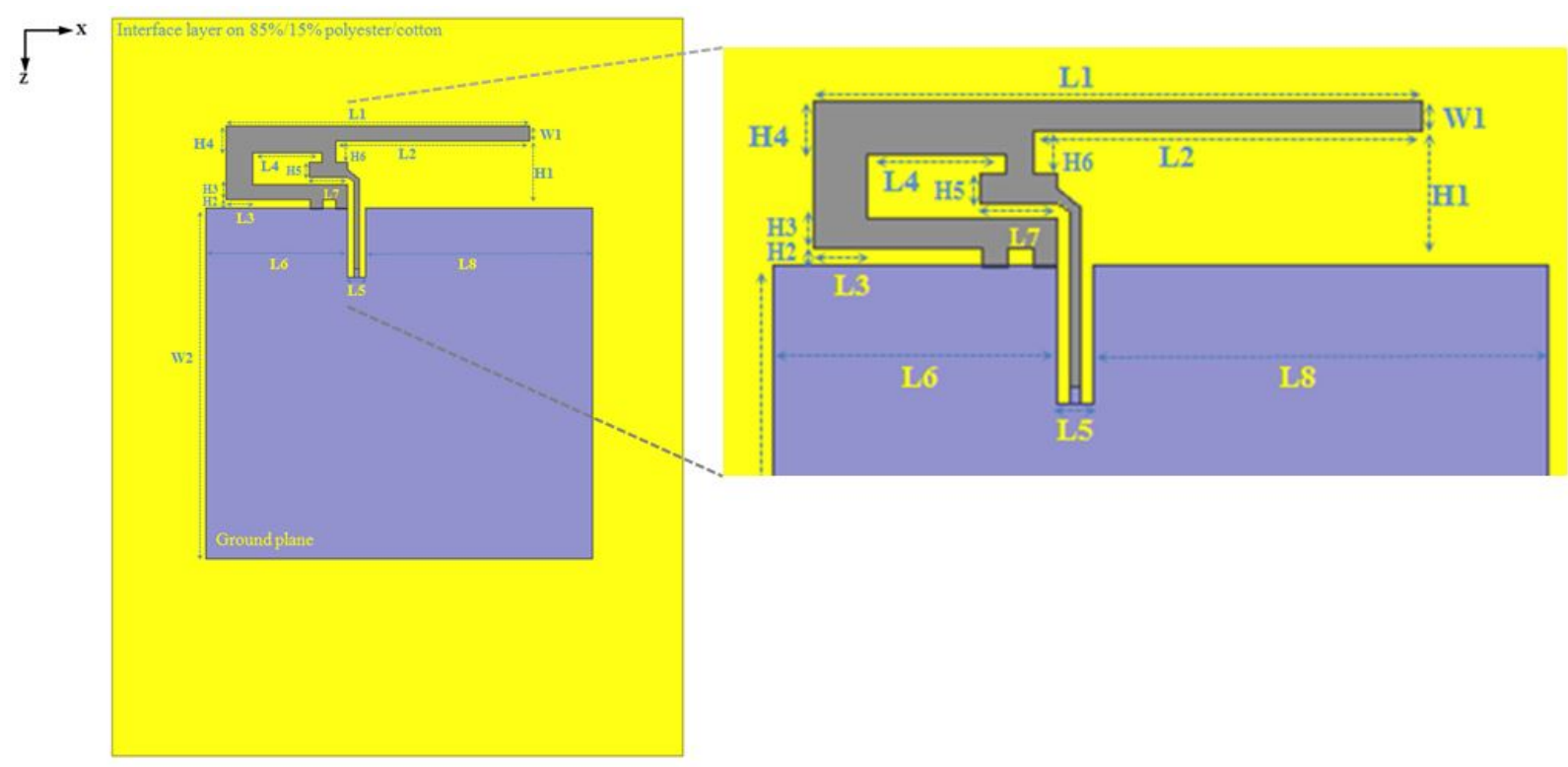

Figure 4.5: Localization circuit's antenna design and dimensions in HFSS

The $2.4 \mathrm{GHz}$ resonant frequency of the IFA on Fig 4.5 is tuned by varying the length of the L2 antenna beam. The ground plane, which is controlled by L5, L6, L8 and W2 dimension, is crucial to the establishment of an adequate impedance matching of the antenna.

Table 4.1: Inverted F-antenna parameters simulated in HFSS

\begin{tabular}{|l|c|c|c|c|c|c|c|c|}
\hline antenna parameters & H1 & H2 & H3 & H4 & H5 & H6 & W1 & W2 \\
\hline dimensions in mm & 5.7 & 0.74 & 1.29 & 2.21 & 1.21 & 1.8 & 1.21 & 29.46 \\
\hline antenna parameters & L1 & L2 & L3 & L4 & L5 & L6 & L7 & L8 \\
\hline dimensions in mm & 25.58 & 16.3 & 2.18 & 5.8 & 1.47 & 11.43 & 3.2 & 19.05 \\
\hline conductivity (MS/m) & 1.86 & \multicolumn{1}{|c|}{ dielectric constant } & 2.57 & loss tangent & 0.019 \\
\hline
\end{tabular}

Using the dimensions from HFSS simulation, the IFA antenna was printed on the $85 \% / 15 \%$ polyester/cotton textile substrate using the Interface Layer method. The antenna is constructed by printing six conductive layers in three printing and thermal curing cycles at $130^{\circ} \mathrm{C}$ for 20 minutes. The resulting antenna thickness is $9 \mu \mathrm{m}$. Additional printing details are fully described in Chapter 3. The fabricated antenna is shown on Fig 4.6(a), It can be seen that antenna is connected to a surface mount 
coaxial connector that is RF compatible with the nominal characteristic impedance

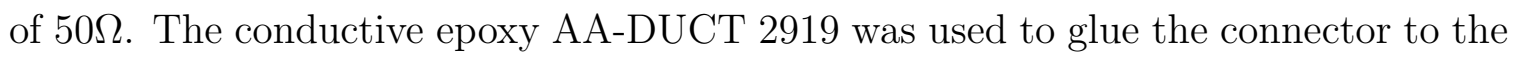
antenna feeding point. Neither the connector nor the epoxy joints were included in the simulation.

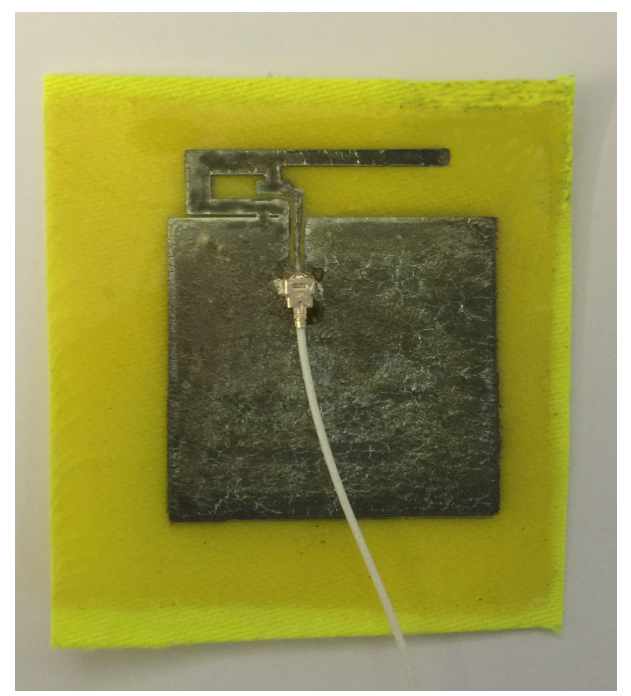

(a)

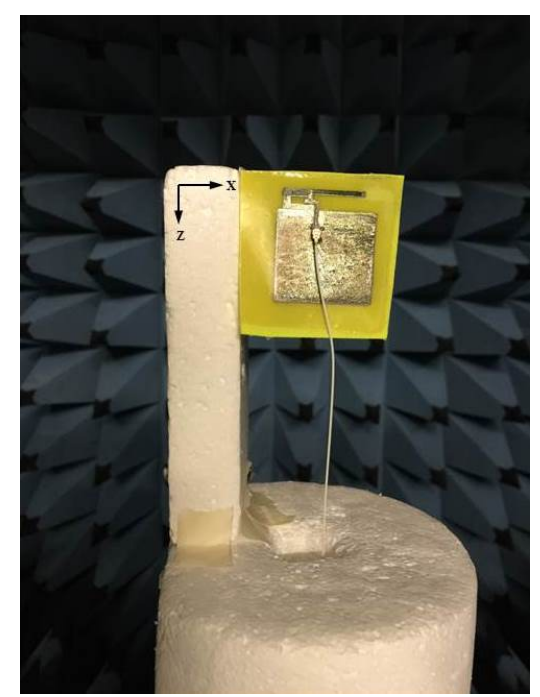

(b)

Figure 4.6: Fabricated IFA a) Front view with connector cable b) Antenna placed in anechoic chamber

The fabricated antenna's reflection coefficients $\left(S_{11}\right)$ were measured using Keysight N9923A FieldFox Vector Network Analyzer. Fig 4.7 plots the reflection coefficients of simulated and fabricated antennas. The similarity between graphs is apparent, and graphs are well matched at $2.4 \mathrm{GHz}$. The fabricated antenna is even more pronounced with very low $S_{11} \approx-20 \mathrm{~dB}$ at $2.4 \mathrm{GHz}$ and bandwidth of nearly $300 \mathrm{MHz}$ when compared to HFSS simulation with $-14 \mathrm{~dB}$ return loss at $2.4 \mathrm{GHz}$ and bandwidth of approximately $250 \mathrm{MHz}$. When the fabricated antenna is placed on a human body, the impedance matching is predictably decreasing to $-13.57 \mathrm{~dB}$ while the bandwidth is comparable to the bandwidth of HFSS simulation and equals to $270 \mathrm{MHz}$. It is clearly seen near the graph edges that the fabricated antenna experiences slight loss of approximately $1 \mathrm{~dB}$ that can be attributed to such unaccounted factors as the 
connector, gluing epoxy, surface roughness, etc.

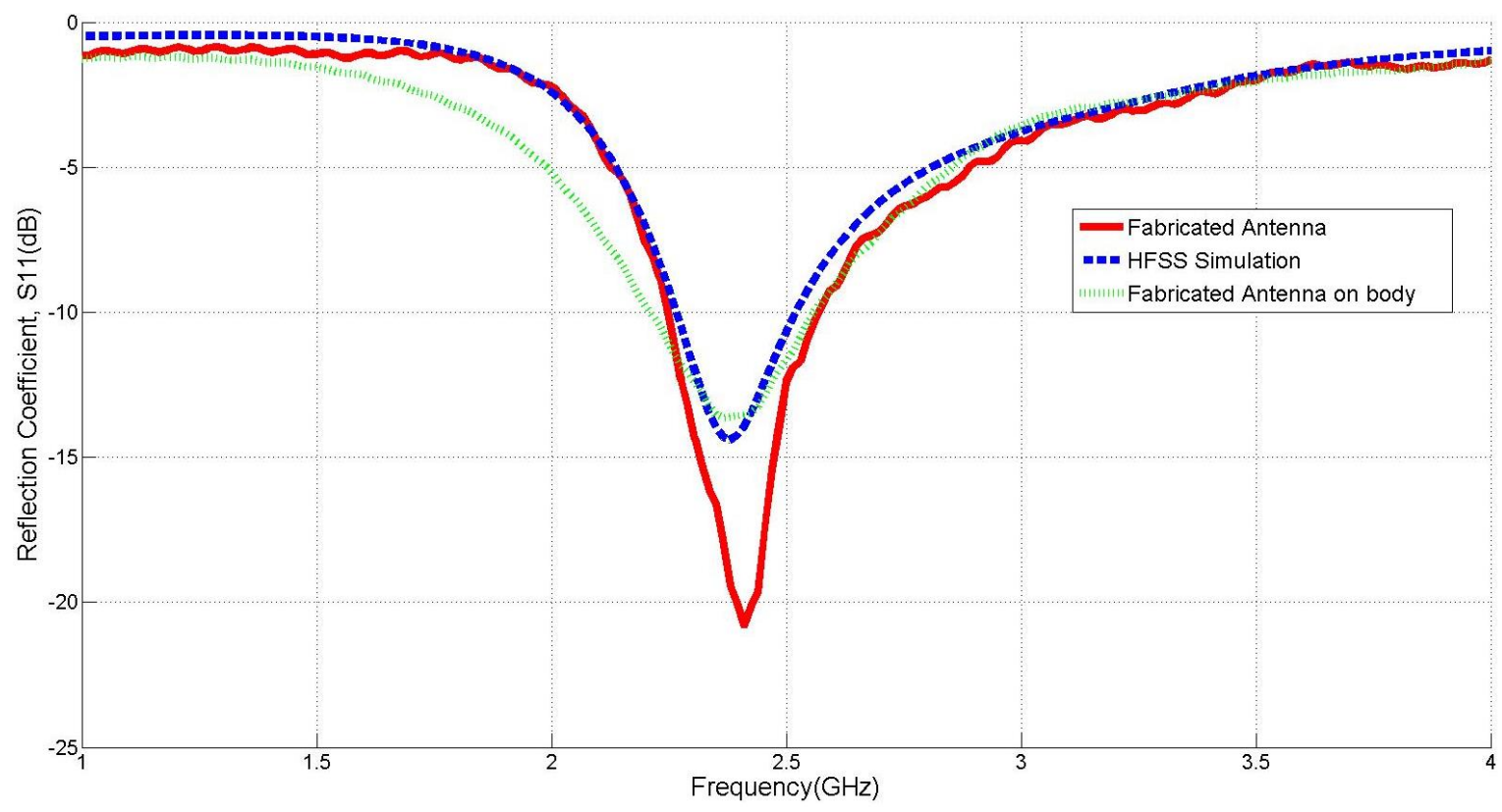

Figure 4.7: Reflection coefficients, S11, of fabricated antenna and HFSS simulation

A 3D radiation pattern of fabricated antenna was measured in Satimo's Star Lab anechoic chamber as shown in Fig 4.6(b), Radiation patterns for both HFSS simulation and fabricated antenna are presented on Fig.4.8. It can be seen that the radiation patterns have similar shapes and comparable concavities along the same directions. Both radiation shapes demonstrate the resemblance to the omni-directional behavior. However, the HFSS simulation estimates the total gain as $2.58 \mathrm{~dB}$ whereas the actual fabricated antenna exhibits the total gain of $1.92 \mathrm{~dB}$. The difference of $0.56 \mathrm{~dB}$ can stem from different factors such as non-ideal fabrication process, the presence of the connector cable that was not accounted for in the simulation, the influence of support structure in an anechoic chamber, etc. 


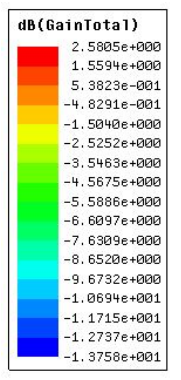

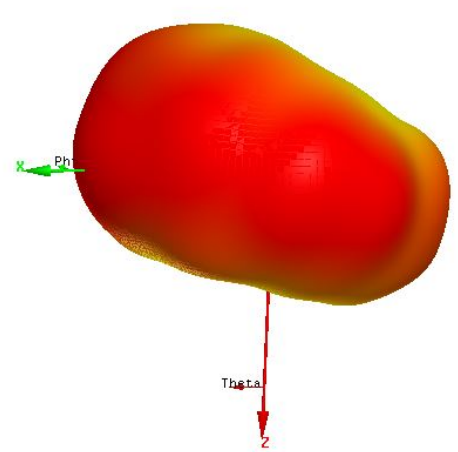

(a)

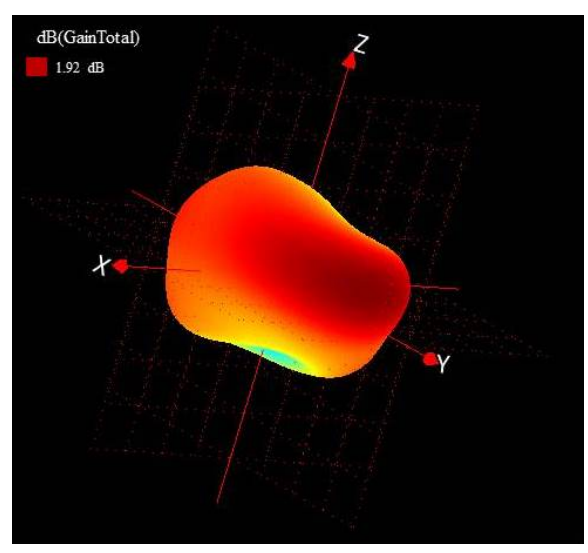

(b)

Figure 4.8: Radiation pattern of a) HFSS simulation b) fabricated antenna

The 2D polar plots of the radiation patterns shown on Fig 4.9 indicate that there is a strong correspondence between the fabricated antenna and HFSS simulation.

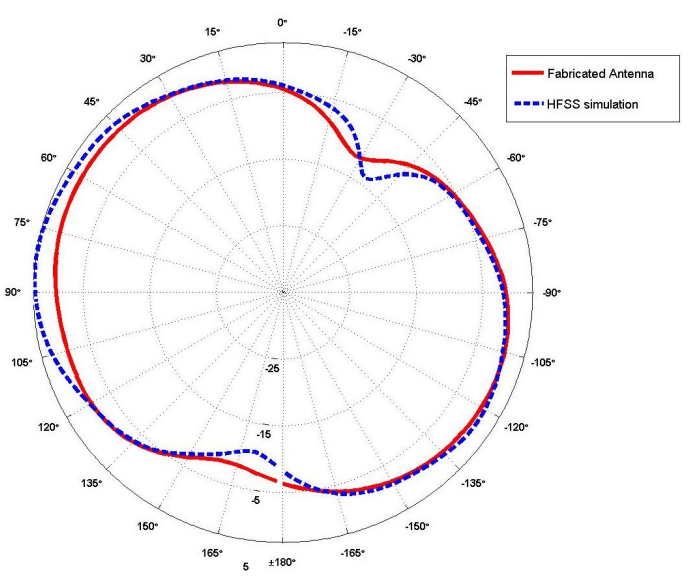

(a)

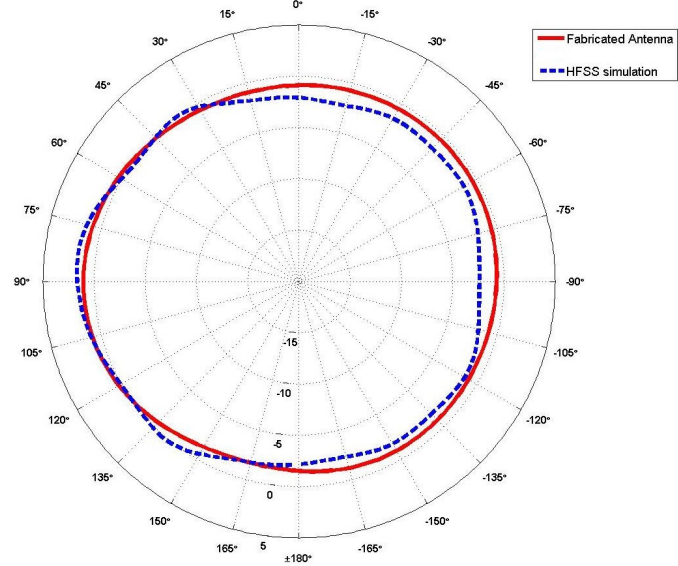

(b)

Figure 4.9: Radiation pattern cross-section of a) E-plane b) H-plane

The H-plane (XY) shown on Fig 4.9(b) exhibits approximately uniform gain across all radiation directions whereas the E-plane (XZ) shown on Fig.4.9(a) has nulls at $-30^{\circ}$ and $+165^{\circ}$. The radiation cross-sections are very similar to the standard dipole patterns with the only difference in the E-plane shift. This shift can be attributed 
to the current flowing in the lower beam shown on Fig 4.10 . This beam is the deviation from the standard inverted 'F' design that was shown on Fig, 4.4 and is used to lengthen the left antenna arm. Such modification is introduced to increase the overall serial inductance that allows better antenna matching while preserving small dimensions. However, this additional detail is the path for current to flow and radiate. This additional current path is essentially an antenna that interferes with the main radiation pattern shifting the E-plane. Nevertheless, the H-plane is still omni-directional, thus the antenna performance is not heavily affected.

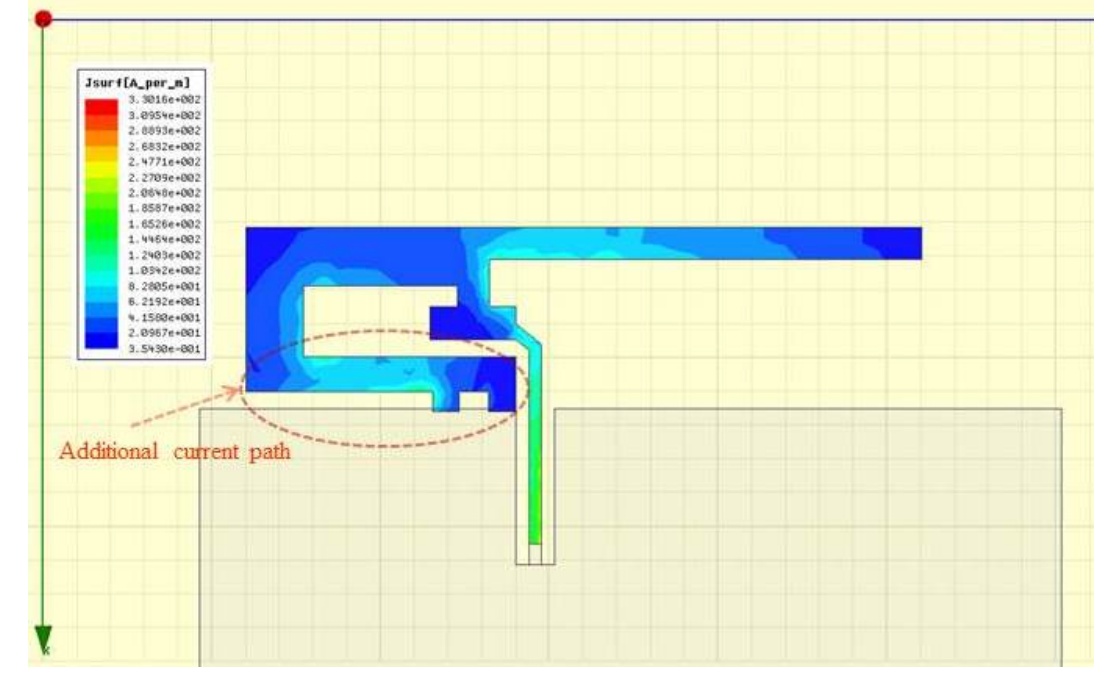

Figure 4.10: Additional current path contributed by extra beam

The fabricated antenna characterization summary is given in Table 4.2. This inverted F-antenna was matched for the use with the interface layer at $2.4 \mathrm{GHz}$ providing $1.82 \mathrm{dBi}$ gain. Thus, it can be incorporated on the $85 \% / 15 \%$ polyester/cotton for the on-textile localization circuit design.

Table 4.2: Fabricated on-textile IFA parameters

\begin{tabular}{|c|c|c|c|c|c|}
\hline Frequency & Return loss & Gain & BW & BW on body & Input imp. \\
\hline $2.4 \mathrm{GHz}$ & $20 \mathrm{~dB}$ & $1.92 \mathrm{dBi}$ & $300 \mathrm{MHz}$ & $270 \mathrm{MHz}$ & $50 \Omega$ \\
\hline
\end{tabular}




\subsubsection{On-Textile Prototype}

Once the antenna has been optimized for the textile substrate, the only thing to consider is the decreasing the number of components to a functional minimum to simplify the final tracking device. The Eagle CAD schematic and the circuit layout are given on Fig.4.11. Recognizing that all interconnects, ground and the antenna will be ink-jet printed, here is the final list of essential components:

- The RN171 WiFi module and Atmega328p microcontroller are main components that were described in the previous section.

- The LED indicates the operational status of the tracking device.

- The oscillator provides the working frequency of $16 \mathrm{MHz}$ to the microcontroller

- The reset resistor is used to prevent the microcontroller from restarting itself.

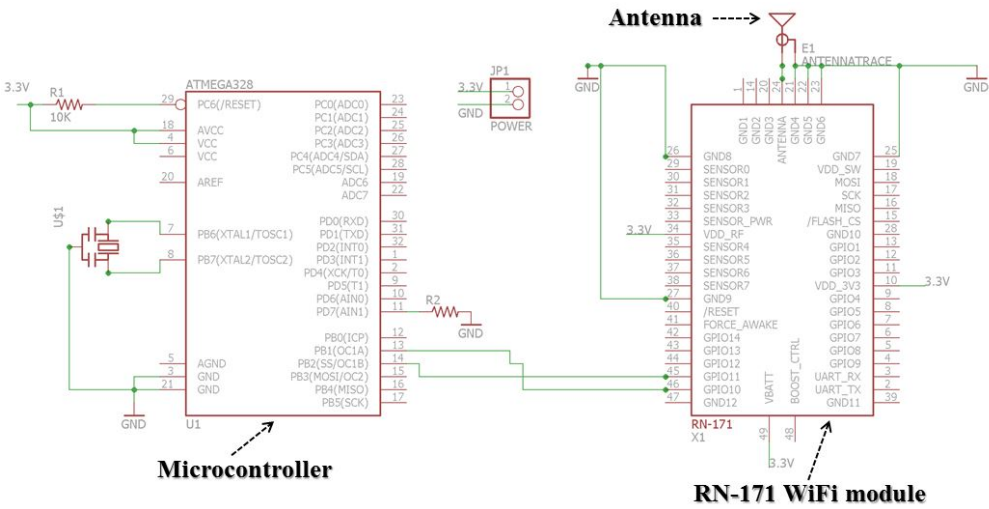

(a)

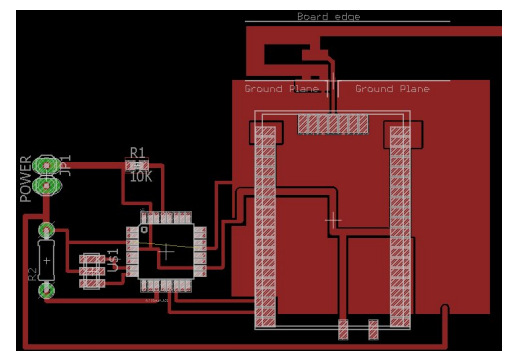

(b)

Figure 4.11: The final localization circuit design a) schematic b) layout 
Once the final circuit is finalized, the on-textile fabrication can be commenced. The following steps describe the production process:

- The circuit layout that is shown on Fig 4.11(b) is conductively ink-jet printed on the interface layer to produce the printed circuit presented on Fig 4.12(a), Conductive printing is accomplished via three printing and sintering cycles to produce six silver layers. The printing time heavily depends on the number of nozzles that are used by the printer. Using five nozzles will lead to the average production time of three hours. However, due to the often clogging of the nozzles, the deposition accuracy may be degraded if high nozzle number is used. Proper nozzle cleaning cycles should be administered to keep nozzles operational.

- The traditional soldering cannot be employed to bond the components to the printed circuit layout. Instead, the conductive epoxy should be used as it is shown on Fig 4.12(b), AA-DUCT 2919 conductive epoxy from Atom Adhesives is used because of its elasticity properties that are useful in flexible circuits such as circuits on textile.

- However, the conductive epoxy alone is not enough to establish strong bonding between big components such as RN171 and the interface layer. Also, without additional attachment, the conductive bonding is easily broken when the textile substrate is bend. A regular epoxy is used for additional fastening of components to the substrate as represented on Fig $4.12(\mathrm{~b})$. For this research 5 -minute epoxy from Devcon is applied but any epoxy can be used as well. 


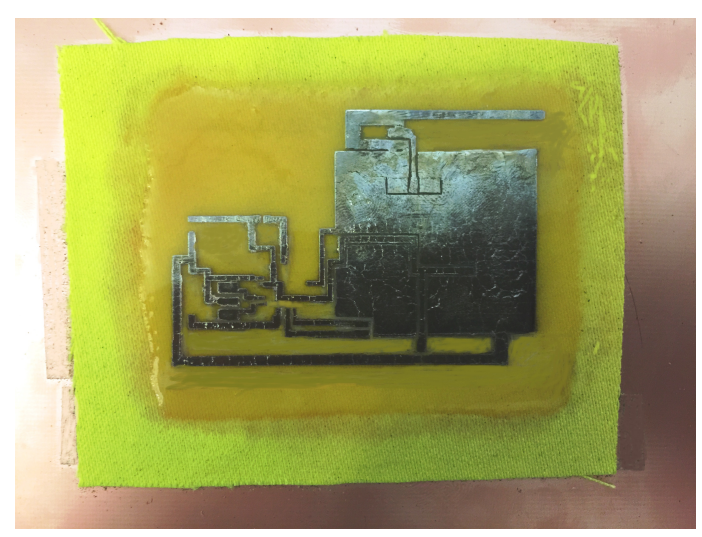

(a)

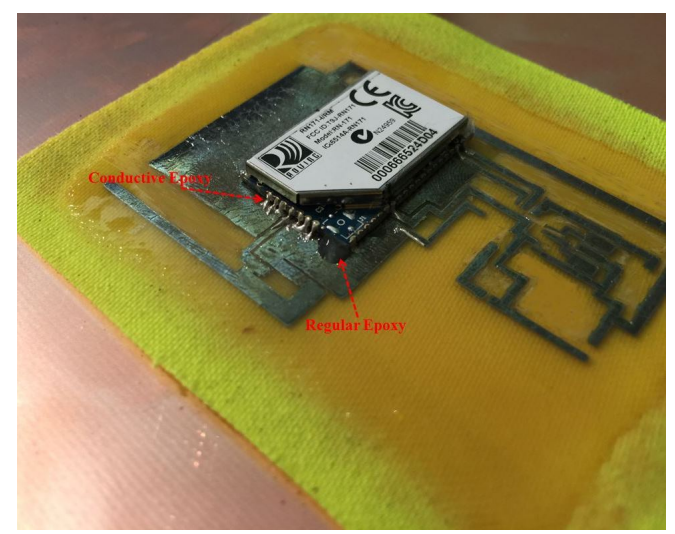

(b)

Figure 4.12: Tracking device fabrication process a) printing circuit layout b) components attachment

Once all components are attached to the printed circuit board, the on-textile prototype is assembled (Fig 4.13). This textile prototype is slightly different from the final localization device. It uses the Atmega328p in DIP package and also DIP socket whereas the final circuit will use the Atmega328p in TQFP package. The final localization device is presented on Fig 4.24 in section 4.4. Such change was administered to facilitate the testing of the on-textile prototype. The socket usage allows for unintrusive troubleshooting, debugging and firmware updating. Therefore, the prototype shown on the Fig 4.13 will be employed solely for the field tests. The prototype is powered by 3.7V Lithium Ion Polymer(LiPo) battery. The battery's capacity is $500 \mathrm{mAh}$ and it can last up to 10 hours at average current consumption of $50 \mathrm{~mA}$ of one transmit and receive call per second. Depending on the number of transmit and receive calls the battery lifetime can be dramatically improved. Also, the battery shown on Fig 4.13 can be replaced with a smaller battery, thus reducing the overall size but decreasing device's battery operation time. The battery shown on Fig 4.13 is rechargeable. Its physical dimensions are $29 \mathrm{~mm} \times 36 \mathrm{~mm} \times 4.75 \mathrm{~mm}$ and weight is 10.5 gram. In the final circuit design supply cables and the battery will be concealed as it is shown on Fig 4.24 . 


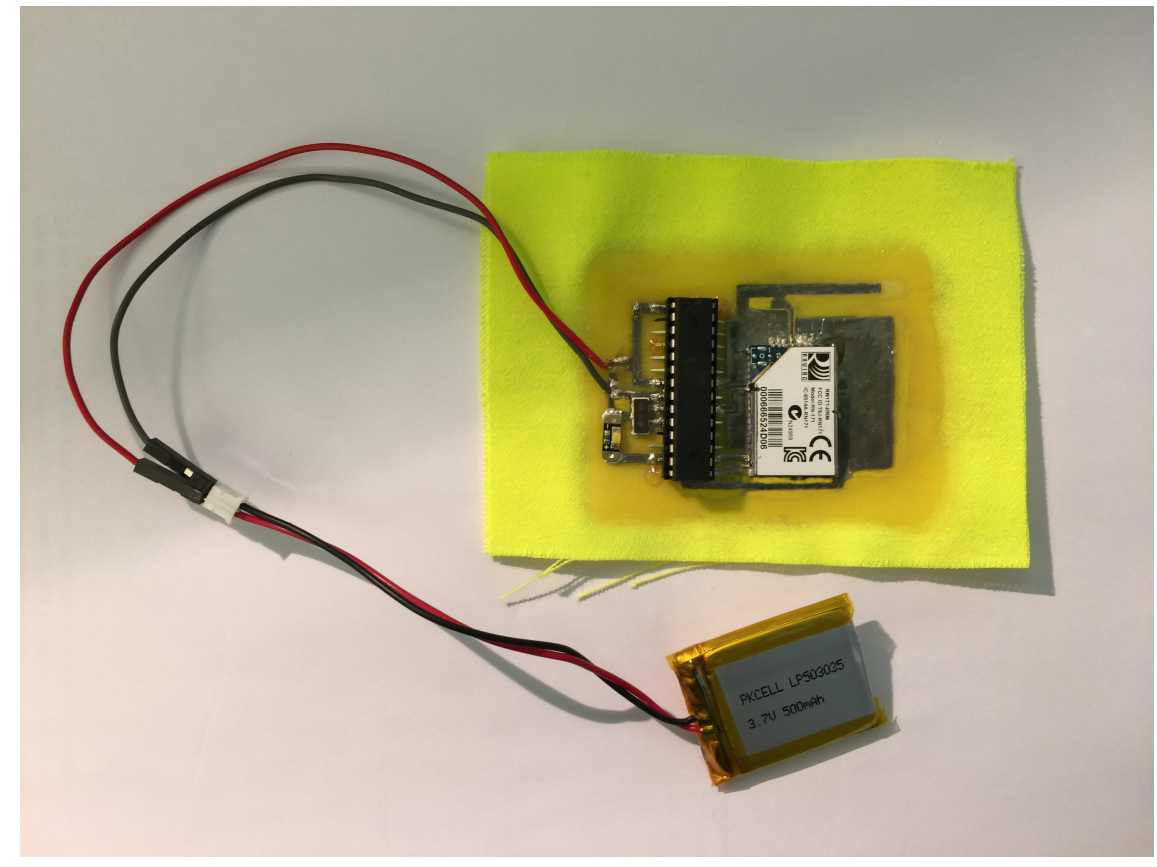

Figure 4.13: On-textile prototype for field tests powered from LiPo battery

\subsection{Field Tests}

Field tests are usually administered to confirm that a device operates correctly. The operation of the on-textile localization prototype developed in the previous section will be tested both in free space (air) and on a human body. It is difficult to simulate accurately the influence of a human body on the localization performance, therefore the field tests are needed to showcase that the localization circuit remains fully functional while being worn.

\subsubsection{Device Radiation Pattern}

Device radiation pattern measurements fall under active antenna testing that is used to confirm that an antenna is functional when incorporated into the final circuit and fed from the microcontroller. During the test, the E-plane and H-plane of the antenna are measured. Since it is an active testing, the real world conditions should be simu- 
lated, thus the measurements were not carried out in an anechoic chamber. Instead, manual measurement process, shown in Fig 4.14, is employed. It is worth noting that the manual radiation pattern measurement only provides a rough estimation of antenna radiation patterns.

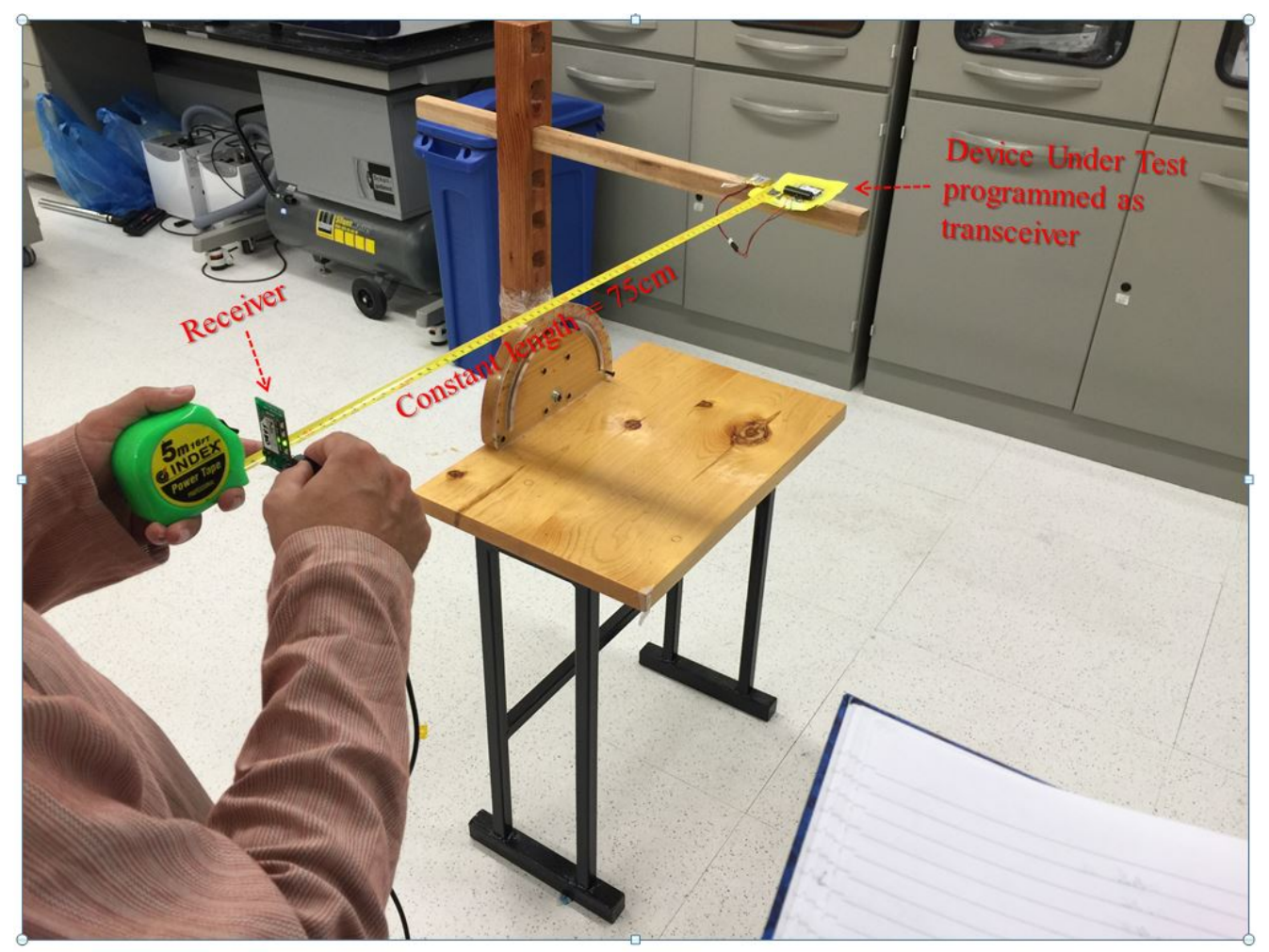

Figure 4.14: Device radiation pattern measurements

The experiment is carried out using transceiver and receiver. The tracking device or Device Under Test (DUT) is programmed as a transceiver, and RN171 evaluation board is configured as a receiver and connected to the laptop. The combination of receiver and laptop is capable of reading the signal transmitted from the DUT. 


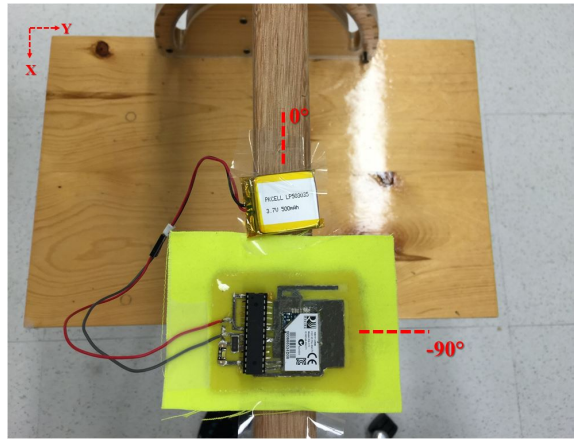

(a)

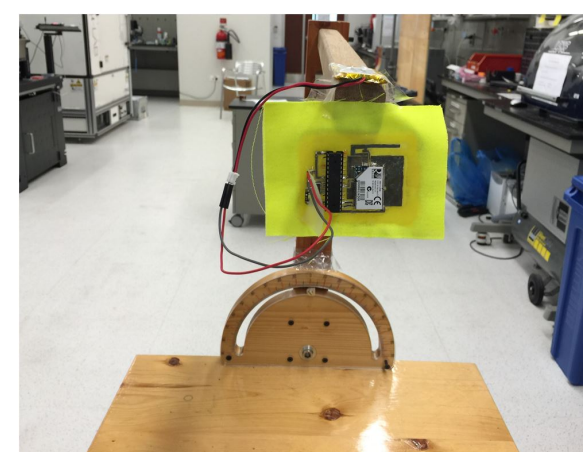

(b)

Figure 4.15: Radiation pattern measurements in free space a) E-plane b) H-plane

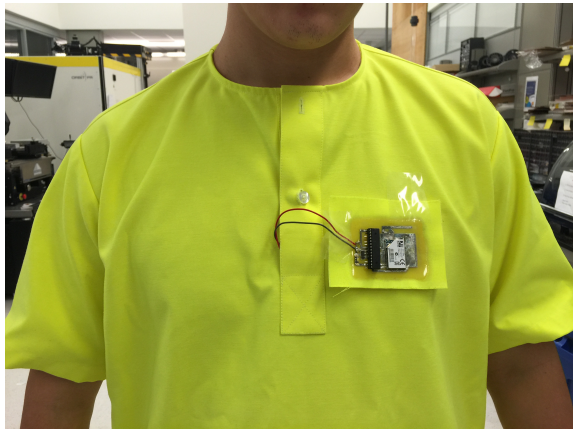

(a)

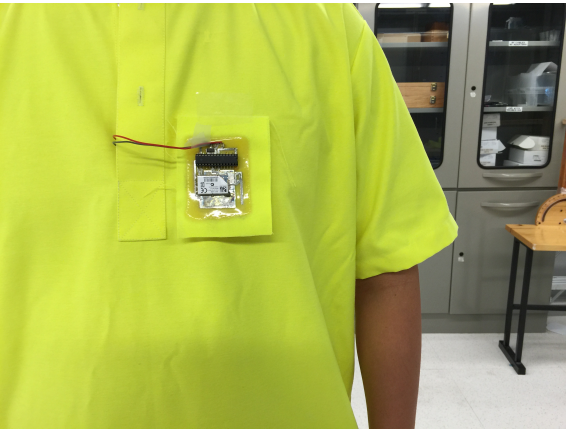

(b)

Figure 4.16: Radiation pattern measurements on body a) E-plane b) H-plane

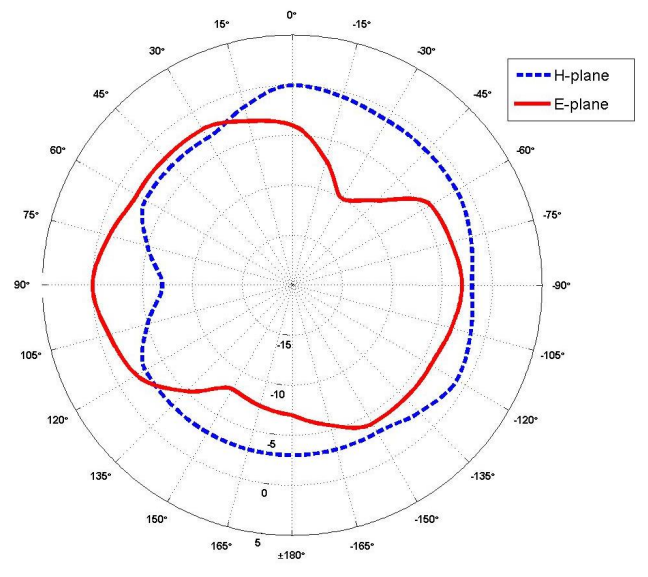

(a)

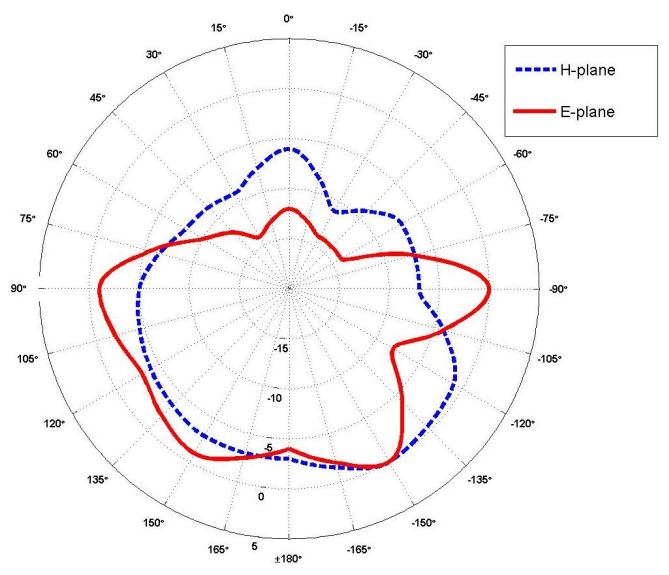

(b)

Figure 4.17: Measured E-plane and H-plane on a) free space b) body 
The DUT provides $12 \mathrm{dBm}(16 \mathrm{~mW})$ of maximum transmitted RF power, whereas receiver's sensitivity is $-83 \mathrm{dBm}$. The device is kept stationary while the receiver is rotated around the DUT in $15^{\circ}$ steps. At each point the RSSI measurement was recorded. The radius of the rotation circle should be in the far-field and should always be constant. For this test, the radius of $75 \mathrm{~cm}$ was selected. Naturally, all rotations will occur in $\mathrm{X}-\mathrm{Y}$ plane (walking on the floor) with the polar coordinate angles as specified on Fig 4.15(a), This exact arrangement will be used in E and $\mathrm{H}$ plane measurements for both free space and on-body tests. The measurement results are normalized with respect to the lowest measurement (because RSSi values are negative).

For free space, E-plane measurement arrangement is represented on Fig 4.15(a) and H-plane measurement setup is shown on Fig 4.15(b). The measured E-plane and H-plane in free space are represented on Fig 4.17(a), The H-plane can be considered omnidirectional with small variation of $7 \mathrm{~dB}$, mainly in the $90^{\circ}$ direction. The embedded circuitry that is located in the same direction may be the reason behind the variation. The E-plane is very similar to the E-field measured during the anechoic characterization of the standalone antenna.

For on-body testing, the DUT is attached to the T-shirt worn by a consenting human. The rest of the experiment is carried out in the same fashion as for free space. E-plane measurement device placement is presented on Fig 4.16(a) and Hplane measurement setup is shown on Fig 4.16(b). The evaluated E-plane and H-plane on the body are represented on Fig 4.17(b). It can be seen that both E and H planes are heavily affected in the direction of human body location ( $90^{\circ}$ to $0^{\circ}$ to $-90^{\circ}$ region) while the opposite direction $\left(90^{\circ}\right.$ to $180^{\circ}$ to $\left.-90^{\circ}\right)$ exhibits the variation in the radiation pattern of $-7 \mathrm{~dB}$. Such phenomenon can be explained by the absorption of radiation by a human body. The human body consists mainly of water and other organic tissues that possess very high dielectric constants and loss tangents. Thus, the human 
body tends to absorb the radiation that clearly can be seen on Fig 4.17(b) where the radiation in the wearer's direction is significantly attenuated. As it was shown before, the effect of the human body on the device radiation pattern is investigated using active testing. Since the degradation of the radiation field in the body direction was expected, and the radiation pattern variation in the out of the body direction is adequate and resembles free space radiation, the antenna can be used for E-textiles in wearable applications.

\subsubsection{Communication Distance}

Communication distance measurements are required to quantify the transmitting and receiving range of the device. Similar to the device radiation pattern measurements, the on-textile prototype was programmed as a transceiver with $12 \mathrm{dBm}$ of maximum $\mathrm{RF}$ power and the RN-171 evaluation board was configured as a receiver with the sensitivity of $-83 \mathrm{dBm}$.

The test was administered by placing both the transceiver and receiver on position zero and then moving the transceiver away from the receiver in five-meter steps. At each point the RSSI measurement was recorded. The test was carried out in an open area on the university campus to reproduce actual operational circumstances. Zero and forty meters measurements are shown in Fig 4.18(a) and Fig.4.18(b) respectively. 


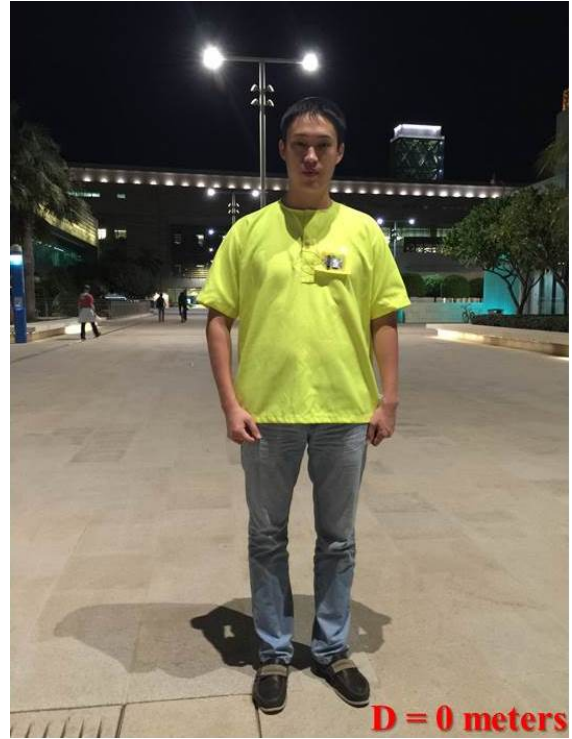

(a)

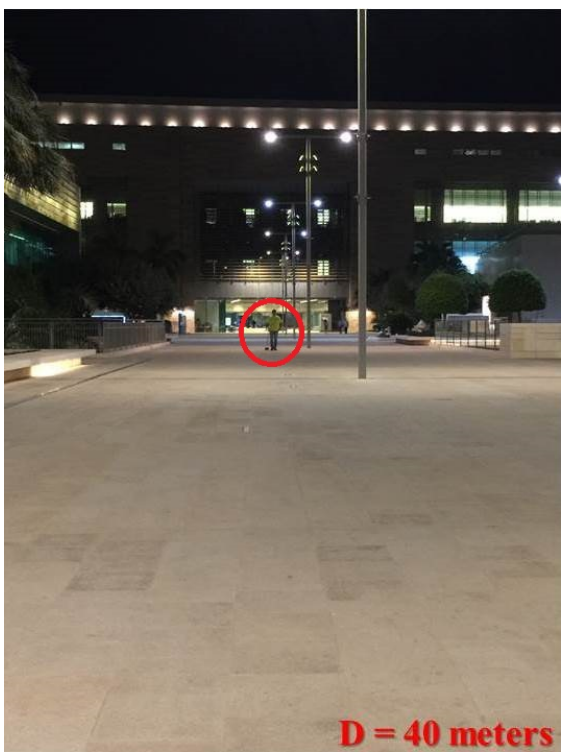

(b)

Figure 4.18: Communication distance measurements on-body at a) 0 meters b) 40 meters

The measurements have been taken both in air and on-body by attaching the device on the T-shirt worn by a consenting human. The measurement results are plotted on Fig 4.19. It turns out that in the air the communication distance of up to 70 meters can be achieved. The distance is reduced to a maximum of 55 meters when the device is tested on the human body. Both of these values are adequate for the targeted applications. It can be seen that with increasing distance the measured RSSI values tend to cluster in -70 to $-80 \mathrm{dBm}$ range that is very close to the receiver sensitivity limit. Thus, improving the receiver sensitivity (for ex. to $-100 \mathrm{dBm}$ ) can easily increase the potential communication distance for both in the air and on-body setups. Nevertheless, the evaluated communication distance of 55 meters is sufficient to read nearby WiFi access points to perform the localization procedure. 


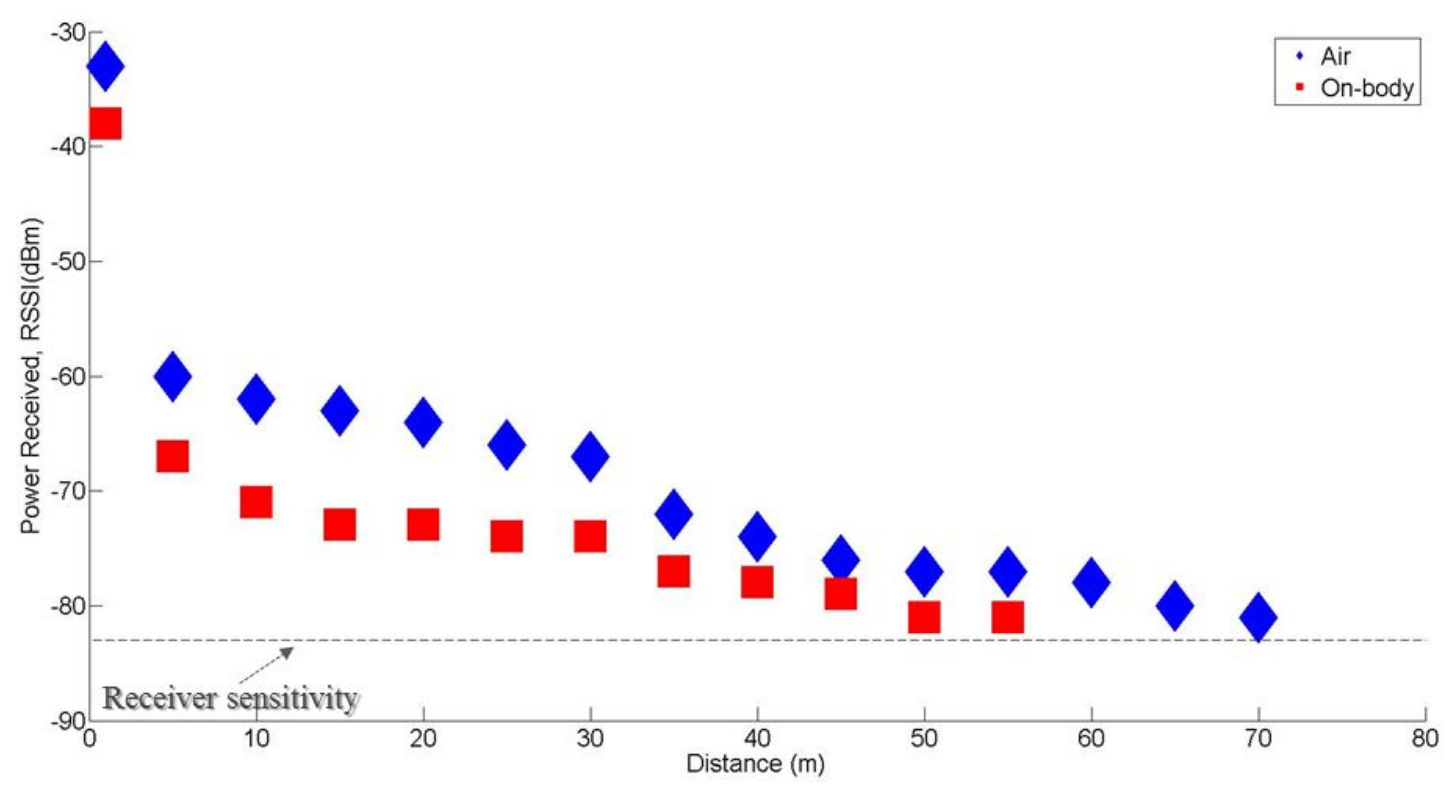

Figure 4.19: Far field communication distance measurement results

\subsubsection{Localization Accuracy}

The main field test for any tracking device is the measurement of the localization accuracy. It is crucial to know whether the tracking device provides accurate position readings. For this test, the tracking device is employed in the normal operation mode. In this mode the localization device scans ten nearby WiFi access points, sends this data to the server that uses Google API to triangulate the position. Finally, the position is plotted on the map that can be accessed from any Internet-enabled device such as laptop or smartphones. Fig 4.20 shows the map with plotted tracking location. Google API returns three values - latitude, longitude and accuracy. The center of the yellow circle ('smart' label) is the estimated device position pinned down by latitude and longitude. The circuit radius from center to red circle outline is a position search region that is constructed by provided accuracy value. Therefore, the Google expects the device to be present somewhere inside the yellow circle (aka search circle). As it will be seen later, the localization accuracy is heavily dependent on Google's WiFi 
access points database and Google's proprietary triangulation algorithms. The access points database is constantly updated when anyone shares his/hers location using a mobile telephone, laptop, etc. Thus, the better localization accuracy expected in more crowded places.

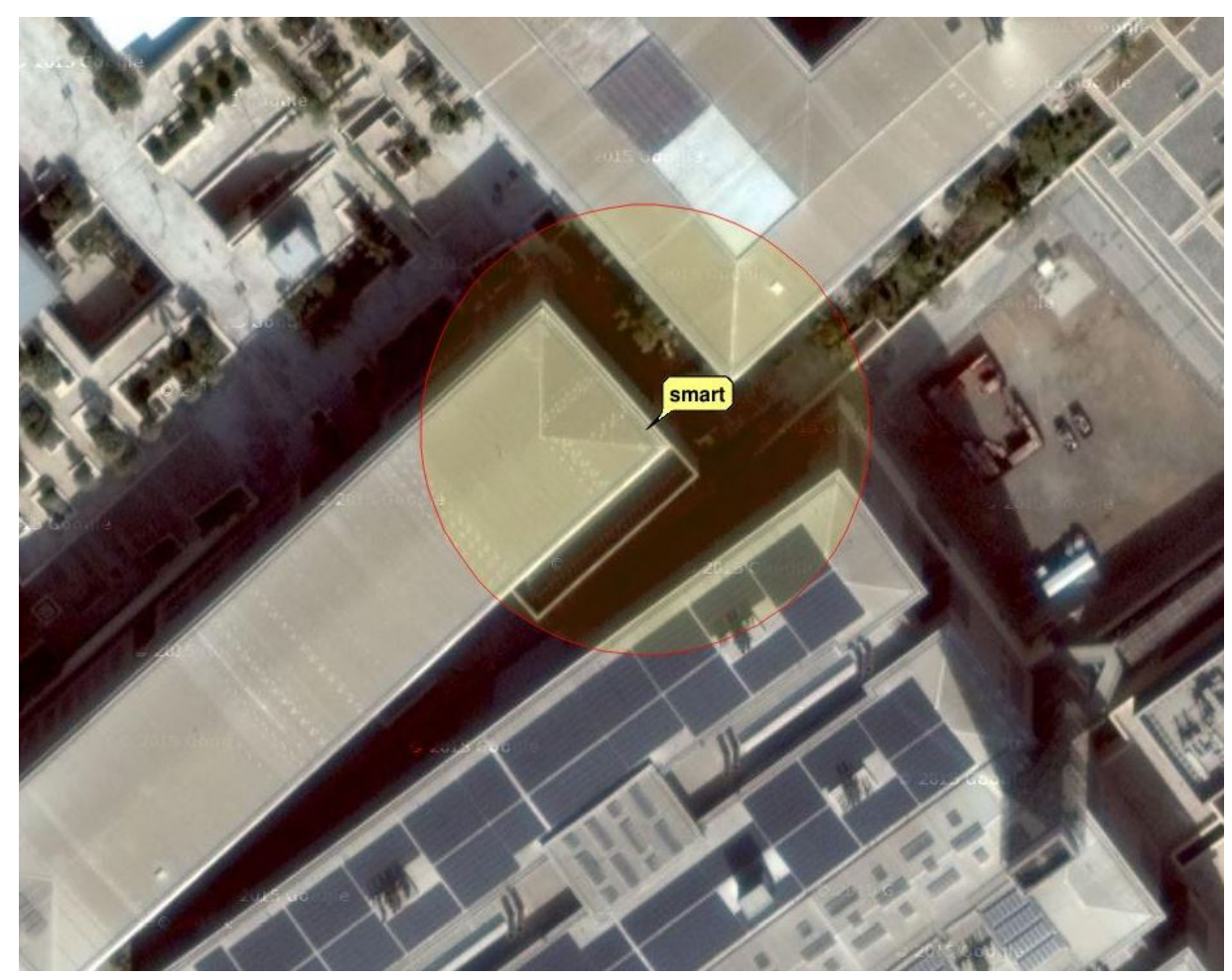

Figure 4.20: Plotting the position of the device on the map

The localization accuracy testing process is carried out using following steps:

1. Ten different locations were selected across KAUST campus. Their latitudes and longitudes were recorded.

2. Human test subject wearing T-shirt with the attached localization device prototype was placed exactly on each of these locations as shown in Fig 4.21 . The position is indicated by the smile face.

3. The position's location was measured via the localization prototype both in air and on body. Estimated latitudes, longitudes and accuracies were recorded, and 
the search circle is plotted on the map as shown on Fig 4.21 .

4. Distance $(d)$ between the original position and the measured position is calculated using formulas (4.1) and (4.2) where $\mathrm{R}$ is the radius of the Earth. These formulas account for the Earth curvature between two latitude and longitude points 67. This deviation distance is indicated as arrow line on Fig.4.20. Moreover, this deviation distance is the actual localization accuracy that is of test's interest.

$$
\begin{gathered}
a=(\sin (\Delta l a t))^{2}+\cos (\operatorname{lat} 1) \cdot \cos (\operatorname{lat} 2) \cdot\left(\sin \left(\frac{\Delta l o n g}{2}\right)\right)^{2} \\
d=2 R \cdot \operatorname{atan} 2(\sqrt{a}, \sqrt{1-a})
\end{gathered}
$$

5. Finally, the original position is checked whether it is inside the search circle or not. The search circle is represented by the yellow circle on Fig 4.21 and its radius is equal to measured accuracy value received from the Google API.

In this test, the deviation distance between the actual position and measured one is of the main concern because it indicates the actual tracking accuracy that the on-textile localization device outputs. Also, it is important to establish whether the actual location is inside the search accuracy radius provided by Google API. Being inside the radius indicates that according to Google algorithms the tracking was accurate enough. The test results for five positions (out of ten) are summarized in Table 4.3. 

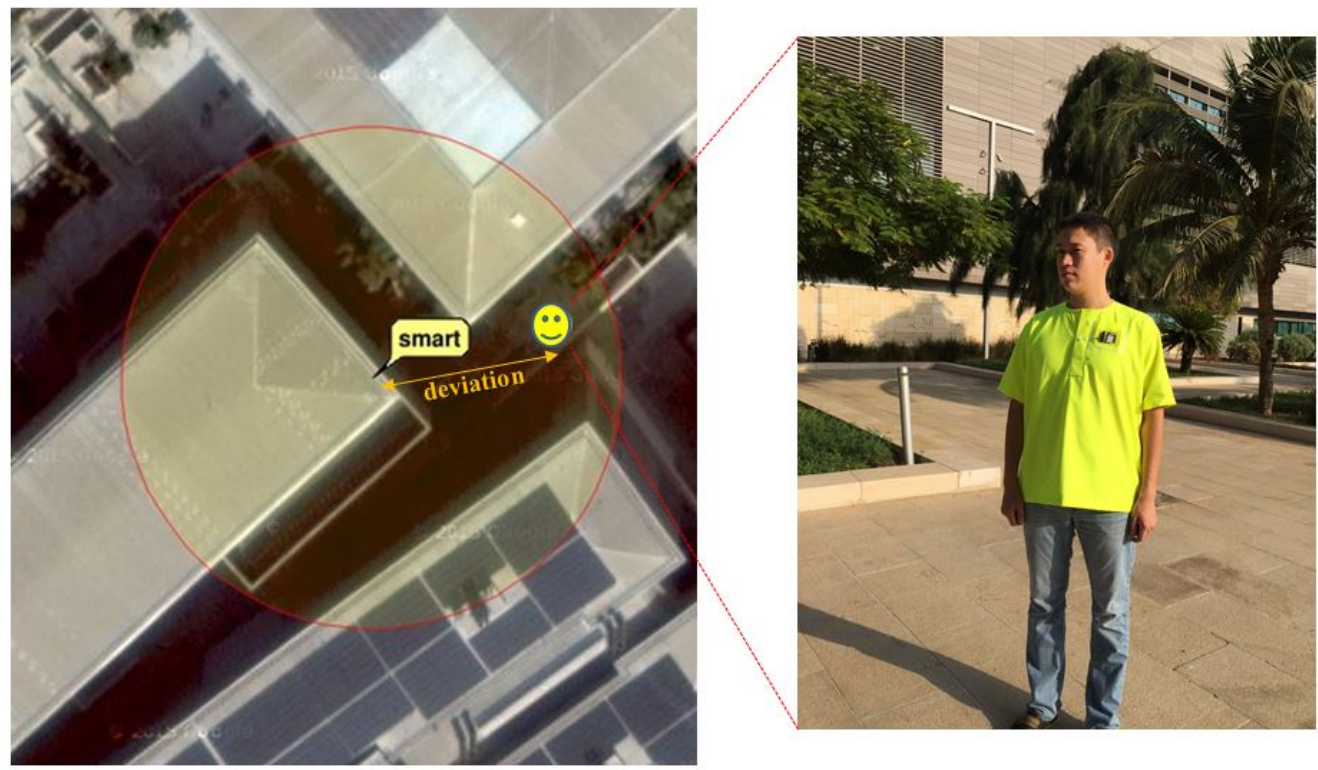

Figure 4.21: Localization accuracy testing process

Table 4.3: Localization accuracy testing results

\begin{tabular}{|c|c|c|c|c|c|c|}
\hline & Position & 1 & 2 & 3 & 4 & 5 \\
\hline \multirow{2}{*}{$\begin{array}{c}\text { Ori } \\
\text { gi } \\
\text { nal }\end{array}$} & $\begin{array}{l}\text { Position } \\
\text { latitude }\left({ }^{\circ}\right)\end{array}$ & 22.307146 & 22.308911 & 22.309901 & 22.309663 & 22.308499 \\
\hline & $\begin{array}{l}\text { Position } \\
\text { longitude }\left({ }^{\circ}\right)\end{array}$ & 39.106044 & 39.105492 & 39.106567 & 39.105446 & 39.104121 \\
\hline \multirow{5}{*}{$\begin{array}{l}\text { In } \\
\text { air }\end{array}$} & Latitude $\left({ }^{\circ}\right)$ & 22.3073012 & 22.3088907 & 22.3099304 & 22.3098656 & 22.3085509 \\
\hline & Longitude $\left({ }^{\circ}\right)$ & 39.1060472 & 39.1056683 & 39.1064914 & 39.1055722 & 39.1041785 \\
\hline & Accuracy (m) & 22 & 74 & 92 & 47 & 20 \\
\hline & Deviation (m) & 17 & 18 & 8 & 26 & 8 \\
\hline & $\begin{array}{l}\text { Within the } \\
\text { search radius? }\end{array}$ & yes & yes & yes & yes & yes \\
\hline \multirow{5}{*}{$\begin{array}{c}\text { On } \\
\text { body }\end{array}$} & Latitude $\left({ }^{\circ}\right)$ & 22.3073385 & 22.3088968 & 22.3099365 & 22.3099429 & 22.3085756 \\
\hline & Longitude $\left({ }^{\circ}\right)$ & 39.1060522 & 39.1056942 & 39.1065042 & 39.1053923 & 39.1042954 \\
\hline & Accuracy $(\mathrm{m})$ & 30 & 75 & 93 & 34 & 35 \\
\hline & Deviation (m) & 17 & 23 & 8 & 25 & 20 \\
\hline & $\begin{array}{l}\text { Within the } \\
\text { search radius? }\end{array}$ & yes & yes & yes & yes & yes \\
\hline \multirow{5}{*}{$\begin{array}{c}25 \\
\mathrm{AP}\end{array}$} & Latitude $\left({ }^{\circ}\right)$ & 22.3073931 & 22.308879 & 22.3101238 & 22.3096563 & 22.3086504 \\
\hline & Longitude $\left({ }^{\circ}\right)$ & 39.1059909 & 39.1056887 & 39.1068614 & 39.1052697 & 39.1041644 \\
\hline & Accuracy (m) & 22 & 76 & 62 & 52 & 49 \\
\hline & Deviation (m) & 28 & 22 & 38 & 20 & 14 \\
\hline & $\begin{array}{l}\text { Within the } \\
\text { search radius? }\end{array}$ & no & yes & yes & yes & yes \\
\hline
\end{tabular}


According to the Table4.3 and Fig 4.23 the distance deviation for in-air and onbody results varies from 8 to 26 meters. Fig 4.22 shows that the average for in air and on body deviation is 15.5 and 18 meters. Thus, there is a 2.5 meters degradation of accuracy for on-body measurements when compared to the in-air results that can be attributed to the shorter transmitting distance of the on-body device. Nevertheless, the on-body localization device is still able to read enough access points to perform the localization process.

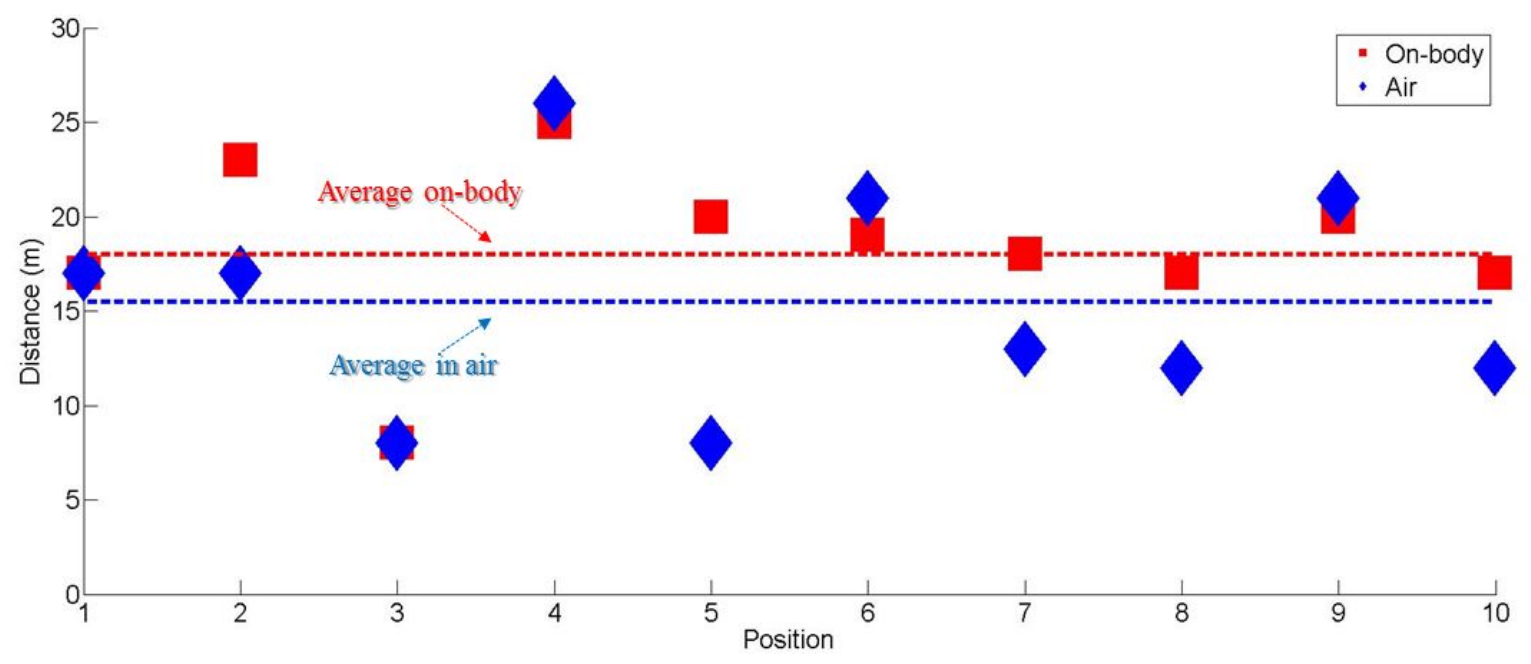

Figure 4.22: The deviation from the original position for on-body and in air measurements

Due to memory limitations the localization device reads data only for ten access points. However, at most locations on KAUST campus up to $25 \mathrm{WiFi}$ access points can be detected. Using RN-171 evaluation board up to 25 access points were recorded for each of the test locations. These data was manually processed through Google API to determine if a higher number of WiFi nodes will improve the localization accuracy. Per Fig 4.23 this prediction did not hold. There is no connection between the number of access points and deviation distance(localization accuracy). Thus, reading ten closest access points is enough to perform the adequate localization. This statement is indirectly seconded by the fact that there was only slight distance 
deviation degradation detected for the on-body testing.

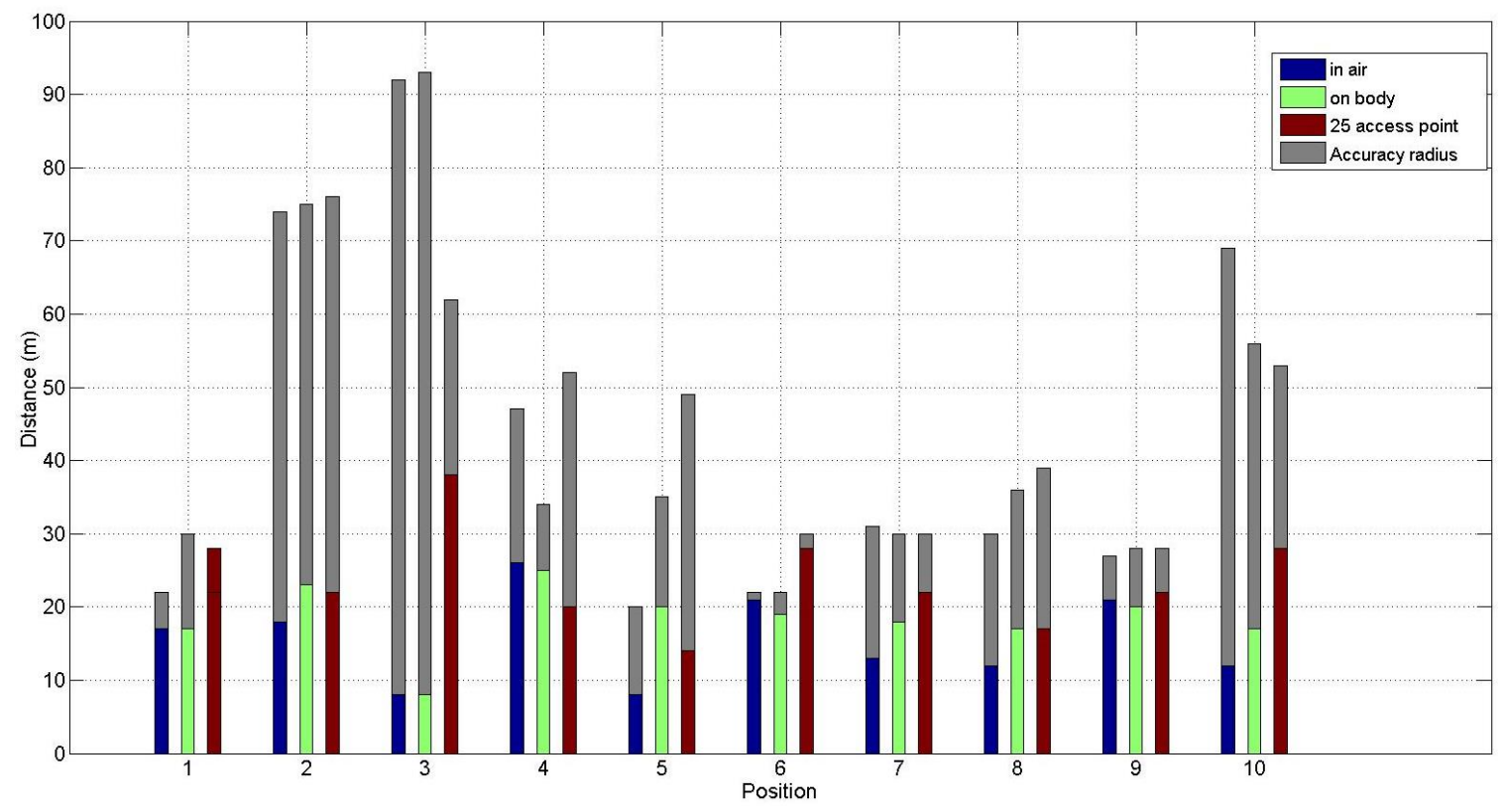

Figure 4.23: Distance deviation between the actual position and the measured position for in air, on body and 25 access points with provided Google accuracy values(grey bars)

It is also clearly represented by grey bars on Fig 4.23 that the original position falls within the Google search radius in virtually all localization measurements. This indicates that according to the Google API the tracking was accomplished successfully.

Of course, the derived localization accuracy of 8-26 meters is not an ideal tracking accuracy. However, the WiFi localization technology is constantly improving due to more access points are being entered in the database and new triangulation algorithms are continuously developed. Nevertheless, the localization accuracy of 8-26 meters is more than enough for a vast number of different applications. Moreover, due to the better filling of Google database in more populous areas, the tracking accuracy of the proposed WiFi localization system can potentially be much better. 


\subsection{Final Device}

The testing results derived in previous sections showcased that the on-textile prototype has acceptable radiation pattern, communication distance and localization accuracy both in free space and when worn on a body. Thus, the on-textile prototype can be turned into the final device by replacing DIP microcontroller and socket with a miniature TQFP package. The final assembled device conductively printed on 85\%/15\% polyester/cotton textile via CM116-20 interface layer is shown on Fig 4.24. The final device can boast a small size of $55 \times 45 \mathrm{~mm}$ and weight of 10 gram without a battery (20.5 gram with battery). The device can be powered from any 3.3-3.7V battery that is rechargeable. Its power consumption is estimated at around $40 \mathrm{~mA}$ in receive $(\mathrm{Rx})$ mode and $180 \mathrm{~mA}$ during transmit $(\mathrm{Tx})$ operation. However, the device remains in transmit or receive mode only for fractions of a second. The current consumption of the whole device was approximated to $50 \mathrm{~mA}$. Thus, the device can be powered from Li-Po $3.7 \mathrm{~V} 500 \mathrm{mAh}$ battery for up to 10 hours. Table 4.4 summarizes the final bill of material for single prototype and bulk quantities including the estimated cost of silver nanoparticle ink, dielectric paste, and epoxies, except the textile cost that varies depending on the fabric type and size. It can be seen that the major cost is contributed by the WiFi module. Replacing the WiFi module with the cheaper alternative will substantially decrease the overall production cost. Bulk costs of ink, paste and epoxies are available only per request.

The additional point worth mentioning is the washability of the circuit. The washability studies have not been carried out and are left for future research. However, to increase the device lifetime when subjugated to washing, the protective polymer paste can be used to conceal the whole device and repel the incoming water. Also, the dry cleaning may be recommended for clothes with the incorporated tracking device. 


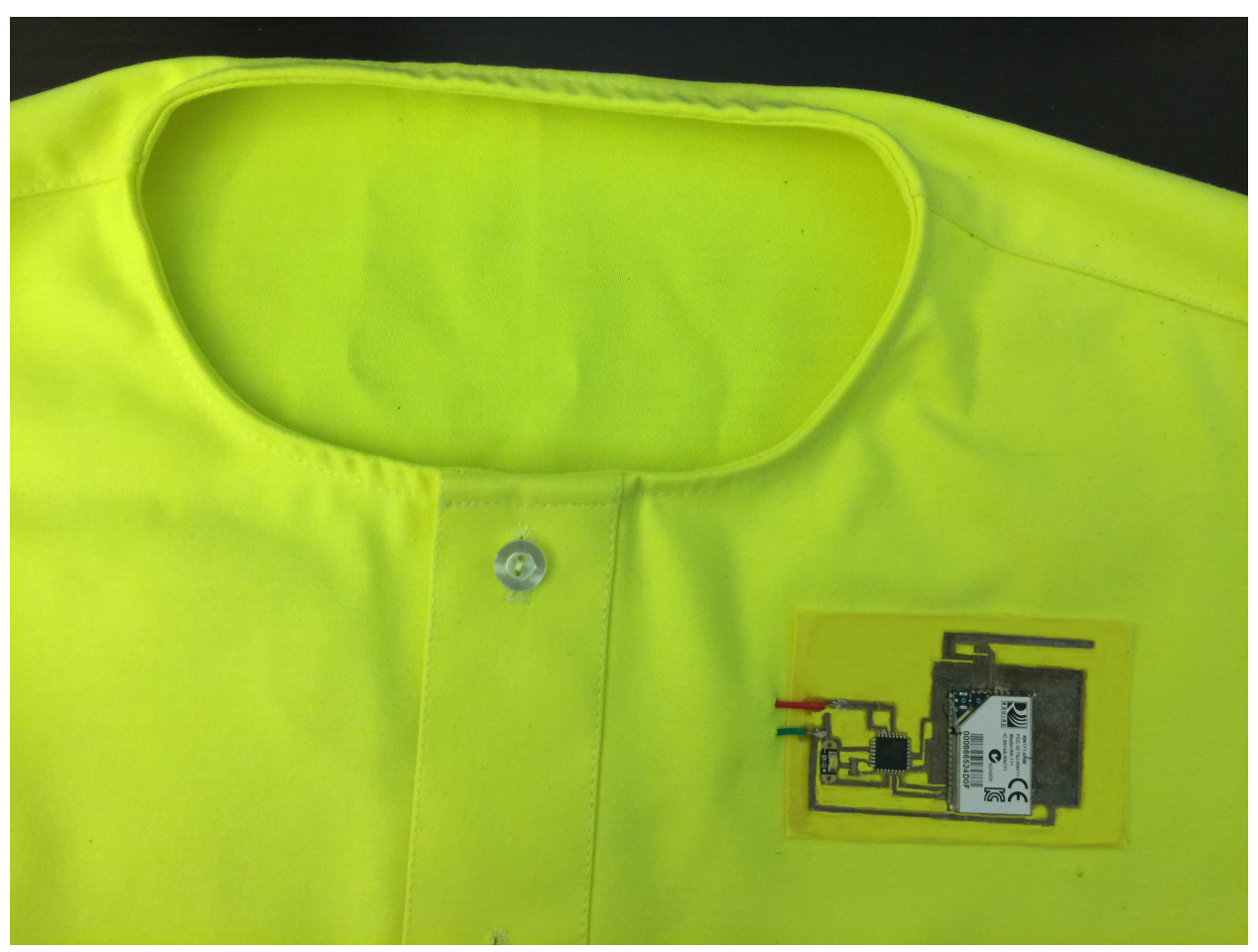

Figure 4.24: The final localization device attached on T-shirt

Table 4.4: Bill of materials of the localization device

\begin{tabular}{|c|c|c|c|}
\hline Material & Required quantity & Cost per prototype (\$) & Cost in bulk (\$) \\
\hline $\begin{array}{l}\text { Atmega328P } \\
\text { TQFP package }\end{array}$ & 1 & 3.38 & 1.69 \\
\hline RN171 & 1 & 26.25 & 20 \\
\hline $16 \mathrm{MHz}$ oscillator & 1 & 0.68 & 0.4 \\
\hline Resistors & 2 & 0.2 & 0.04 \\
\hline LED & 1 & 0.29 & 0.05 \\
\hline $3.7 \mathrm{~V}$ battery & 1 & 5.95 & 4.76 \\
\hline CM116-20 & $5 g$ & 2.5 & per request \\
\hline ANP DGP 40LT & $1 \mathrm{~g}$ & 6 & per request \\
\hline AA-DUCT 2919 & $1 \mathrm{~g}$ & 2 & per request \\
\hline 5 minute epoxy & $1 \mathrm{~g}$ & 0.04 & per request \\
\hline & Total cost: & 47.29 & 37.48 \\
\hline
\end{tabular}

As it was described in Chapter 3, the interface layer formed by CM116-20 dielectric ink is flexible and can be bent several times without any considerable degradation of conductive properties. While remaining relatively flexible as on Fig 4.25, the elasticity 
of the final localization circuit is affected by sizable components such as RN171 WiFi module. This module is big enough to resist the bending attempts. Furthermore, excessive textile curvature can potentially rip apart the bonding between layout and the chip. On the other hand, smaller electronic components are much more resilient to bending. Thus, decreasing the WiFi module size will dramatically increase the overall circuit flexibility.

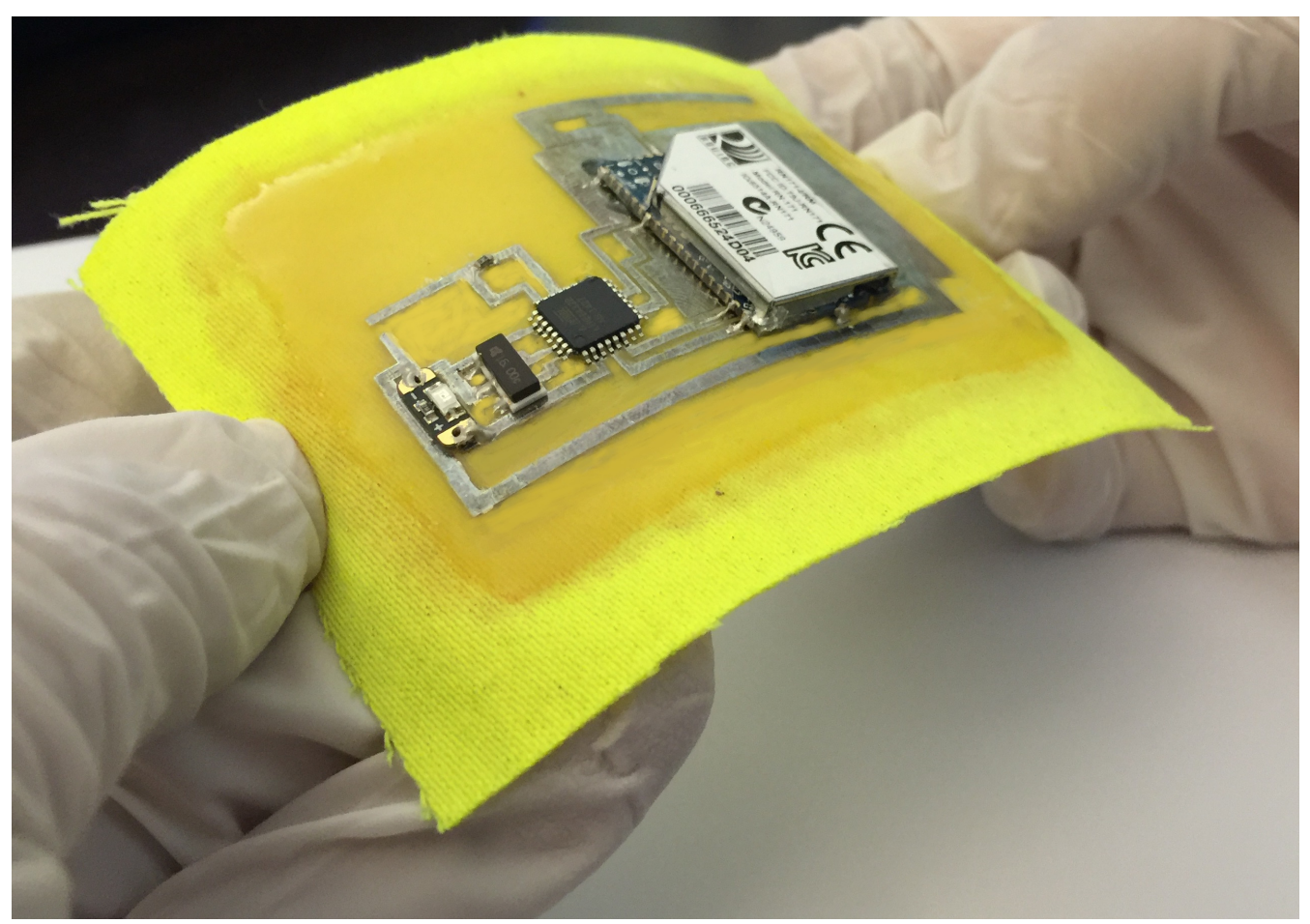

Figure 4.25: The overall flexibility of the final localization device

\subsection{Summary}

This chapter was devoted to the fabrication and testing of the on-textile localization device. Initially, the proposed operation and system diagram were presented. Based on that, the PCB prototype of the localization device was constructed. Through the PCB prototype the microcontroller firmware and server software were debugged and finalized. 
In the next section, the localization prototype was constructed on the textile substrate via the Interface Layer method. In order to accomplish this task, the antenna was optimized to function properly on the textile substrate. As the result, the inverted F-antenna, capable of functioning on the textile substrate at $2.4 \mathrm{GHz}$ outputting $1.92 \mathrm{dBi}$ of total gain, was derived. Then, the on-textile prototype was fabricated that featured only the most essential components required for the test fields.

Finally, the on-textile prototype performance was field tested. The radiation pattern, transmitting distance and localization accuracy were analyzed. Once the test results proved the adequate functioning of the localization prototype, the final device was constructed. The parameters of the final localization device are summarized in Table 4.5.

Table 4.5: The parameters of the localization device build on textile substrate via the Interface Layer method

\begin{tabular}{|l|c|}
\hline Parameter & Value \\
\hline Weight & 10 gram \\
\hline Dimensions & $55 \times 45 \mathrm{~mm}$ \\
\hline Power consumption & $40 \mathrm{~mA}$ in Rx, $180 \mathrm{~mA}$ in Tx \\
\hline Operating frequency & $2.4 \mathrm{GHz}$ \\
\hline Bandwidth & $270 \mathrm{MHz}$ \\
\hline Antenna gain & $1.92 \mathrm{dBi}$ \\
\hline Transmitting distance & up to $55 \mathrm{~m}$ \\
\hline Localization accuracy & up to $8 \mathrm{~m}$ \\
\hline Interface layer material & CM116-20 \\
\hline Silver ink material & ANP DGP 40LT-15C \\
\hline
\end{tabular}

The final localization device is presented on Fig,4.24. It is small, light-weight and flexible. It features, for the first time, the complete circuit layout printed on textile via the Interface Layer method. The employment of the Interface Layer fabrication method makes it potentially easy to reproduce this localization device with standard mass production techniques such as automated screen and ink-jet printing, pick and place manufacturing, etc. Thus, the fabrication process of this localization device 
is very similar to the traditional PCB manufacturing while preserving innate textile flexibility. Finally, the developed device is the first true E-textile WiFi localization gadget that is completely incorporated with regular clothes via conductive printing. 


\section{Chapter 5}

\section{Concluding Remarks}

\section{$5.1 \quad$ Summary}

This thesis describes the creation of the fully functional localization device on textile via the Interface Layer method. The primary contribution of this thesis lies in its multidisciplinary nature because it combines both materials and device research aspects. The materials part is focused on the expansion of scantily explored direct conductive printing via the Interface Layer method. The device is part is focused on the creation of the first true E-textiles tracking device using the conductive printing results developed in the materials part.

The conductive printing via the Interface Layer method has never been accomplished using any other material than the special interface paste Fabink-UV-IF4. However, this ink is seven times more expensive than ordinary dielectric ink. In order to decrease the cost and, consequently, foster the development of the Interface Layer method new interface layer materials should be researched. Thus, in Chapter 3, the interface layer formation process utilizing the general purpose CM116-20 dielectric paste is investigated, and the processing recipe is created. It has been proven that the smooth and flexible interface layers can be formed using CM116-20 and manual screen printing technique. Furthermore, the conductive printing was successfully performed on the CM116-20 interface layer, and its characterization was carried out. 
Next, the CM116-20 characterization results were used to print the complete circuit layout of the developed tracking device on textile via the Interface Layer method. The tracking applications are common in different fields, but there are currently no true E-textile localization devices. And the Interface Layer method can be used for their creation because this method is easily mass producible and it preserves the textile flexibility. In chapter 4 the on-textile tracking device was produced via conductive printing on the CM116-20 interface layer followed by the electronic components attachment. The resulting device was field tested and showed the communication distance up to 55 meters and localization accuracy up to 8 meters while being worn on a body. The developed localization device is flexible and small enough for people to comfortably wear it on clothes such as uniforms, lab coats, nursing gowns, etc. This device is connected to the server, thus its position can be determined from any Internet-enabled device such as a mobile phone or a laptop.

\section{$5.2 \quad$ Future Research Work}

It is strongly believed that the development of E-textiles via the Interface Layer method is still in its infancy, and much more work can be done in this field. The developed localization device is also can be improved in several ways. Thus, the work presented in this thesis can be extended in the following directions.

1. Since the interface layer fabrication time using the general purpose CM116-20 is long, new processing recipes can be researched that will decrease the production time.

2. In this study only the single layered circuit layouts were considered. However, for more complicated devices it will be necessary to print multiple layered circuit designs. The research in this direction is very critical for the development of the E-textile field. 
3. The field tests were used to access the body effect on the antenna radiation performance. However, the better approach that can provide more depth into actual antenna operation is to include the body effect into simulation.

4. Since the tracking device is incorporated with clothes, the issue of cleaning arises. However, no washability testings have been accomplished in this research. The resilience to washing can be improved by covering the circuit with a protective polymer coating. More research needs to be done in this direction. 


\section{REFERENCES}

[1] W. Whittow, A. Chauraya, J. Vardaxoglou, Y. Li, R. Torah, K. Yang, S. Beeby, and J. Tudor, "Inkjet-printed microstrip patch antennas realized on textile for wearable applications," Antennas and Wireless Propagation Letters, IEEE, vol. 13, pp. 71-74, 2014.

[2] A. Chauraya, W. Whittow, J. Vardaxoglou, Y. Li, R. Torah, K. Yang, S. Beeby, and J. Tudor, "Inkjet printed dipole antennas on textiles for wearable communications," Microwaves, Antennas Propagation, IET, vol. 7, no. 9, pp. 760-767, June 2013.

[3] G. Paul, F. Cao, R. Torah, K. Yang, S. Beeby, and J. Tudor, "A smart textile based facial emg and eog computer interface," Sensors Journal, IEEE, vol. 14, no. 2, pp. 393-400, Feb 2014.

[4] W. Whittow, Y. Li, R. Torah, K. Yang, S. Beeby, and J. Tudor, "Printed frequency selective surfaces on textiles," Electronics Letters, vol. 50, no. 13, pp. 916-917, June 2014.

[5] Y. Li, R. Torah, S. Beeby, and J. Tudor, "Fully direct-write dispenser printed dipole antenna on woven polyester cotton fabric for wearable electronics applications," Electronics Letters, vol. 51, no. 17, pp. 1306-1308, 2015.

[6] Y. Wei, R. Torah, K. Yang, S. Beeby, and J. Tudor, "A novel fabrication process to realise piezoelectric cantilever structures for smart fabric sensor applications," in Sensors, 2012 IEEE, Oct 2012, pp. 1-4.

[7] Y. Li, R. Torah, S. Beeby, and J. Tudor, "An all-inkjet printed flexible capacitor for wearable applications," in Design, Test, Integration and Packaging of MEMS/MOEMS (DTIP), 2012 Symposium on, April 2012, pp. 192-195. 
[8] Y. Wei, Y. Li, R. Torah, and J. Tudor, "Laser curing of screen and inkjet printed conductors on flexible substrates," in Design, Test, Integration and Packaging of MEMS/MOEMS (DTIP), 2015 Symposium on, April 2015, pp. 1-4.

[9] Y. Wei, R. Torah, K. Yang, S. Beeby, and J. Tudor, "A novel fabrication process to realise a valveless micropump on a flexible substrate," in Solid-State Sensors, Actuators and Microsystems (TRANSDUCERS EUROSENSORS XXVII), 2013 Transducers Eurosensors XXVII: The 17th International Conference on, June 2013, pp. 1079-1082.

[10] C. Kutzner, R. Lucklum, R. Torah, S. Beeby, and J. Tudor, "Novel screen printed humidity sensor on textiles for smart textile applications," in Solid-State Sensors, Actuators and Microsystems (TRANSDUCERS EUROSENSORS XXVII), 2013 Transducers Eurosensors XXVII: The 17th International Conference on, June 2013, pp. 282-285.

[11] Y. Li, R. Torah, S. Beeby, and J. Tudor, "An all-inkjet printed flexible capacitor on a textile using a new poly(4-vinylphenol) dielectric ink for wearable applications," in Sensors, 2012 IEEE, Oct 2012, pp. 1-4.

[12] Y. Wei, R. Torah, K. Yang, S. Beeby, and J. Tudor, "A novel fabrication process for capacitive cantilever structures for smart fabric applications," in Design, Test, Integration and Packaging of MEMS/MOEMS (DTIP), 2012 Symposium on, April 2012, pp. 136-139.

[13] S. Zhang, R. Seager, A. Chauraya, W. Whittow, and Y. Vardaxoglou, "Textile manufacturing techniques in rf devices," in Antennas and Propagation Conference (LAPC), 2014 Loughborough, Nov 2014, pp. 182-186.

[14] Cisco, "Cisco visual networking index: Global mobile data traffic forecast update 2014-2019," 2014, accessed: 2015-10-23. [Online]. Available: http://www.cisco.com/c/en/us/solutions/collateral/service-provider/ visual-networking-index-vni/white_paper_c11-520862.html

[15] Tractica, "Smart clothing and body sensors: Connected sports and fitness apparel, fashion apparel, baby and pregnancy monitors, heart rate monitors, headbands, posture monitors and 3d trackers," May 2015, 
accessed: 2015-10-23. [Online]. Available: http://www.giiresearch.com/report/ trac329326-smart-clothing-body-sensors-connected-sports.html

[16] I. Locher, "Technologies for system-on-textile integration," Ph.D. dissertation, ETH Zrich, 2006.

[17] A. Derek, "The best kids trackers: Using wearables for child safety," accessed: 2015-10-29. [Online]. Available: http://www.wareable.com/internet-of-things/ the-best-kids-trackers

[18] H. Alan, "Screen printing for the industrial user," accessed: 2015-11-01. [Online]. Available: http://www.gwent.org/gem_screen_printing.html

[19] H. Nurul, F. Malek, P. Soh, G. Vandenbosch, V. Volski, S. Ooi, and I. Adam, "Evaluation of a wearable hybrid textile antenna," in Antennas and Propagation Conference (LAPC), 2010 Loughborough, Nov 2010, pp. 337-340.

[20] Y. P. Huang, "Effect of sewing types on flexible embroidery antennas in uhf band," in Microwave Conference (EuMC), 2013 European, Oct 2013, pp. 88-91.

[21] S. Shin, B. Kim, Y. K. Son, J. E. Kim, and I.-Y. Cho, "A flexible textile wristwatch using transfer printed textile circuit technique," in Consumer Electronics (ICCE), 2012 IEEE International Conference on, Jan 2012, pp. 21-22.

[22] A. Kiourti, C. Lee, and J. Volakis, "Fabrication of textile antennas and circuits with $0.1 \mathrm{~mm}$ precision," Antennas and Wireless Propagation Letters, IEEE, vol. PP, no. 99, pp. 1-1, 2015.

[23] C. Zysset, K. Cherenack, T. Kinkeldei, and G. Troster, "Weaving integrated circuits into textiles," in Wearable Computers (ISWC), 2010 International Symposium on, Oct 2010, pp. 1-8.

[24] M. Varga, N. Munzenrieder, C. Vogt, and G. Troster, "Programmable etextile composite circuit," in Electronic Components and Technology Conference (ECTC) , 2015 IEEE 65th, May 2015, pp. 678-684. 
[25] L. Li, K. Yang, G. Song, L. Zhang, and M. Liu, "Development of knitted fabric sensors for monitoring human respiration," in Electronic Measurement Instruments, 2009. ICEMI '09. 9th International Conference on, March 2015.

[26] H. Kim, Y. Kim, Y.-S. Kwon, and H.-J. Yoo, "A 1.12mw continuous healthcare monitor chip integrated on a planar fashionable circuit board," in Solid-State Circuits Conference, 2008. ISSCC 2008. Digest of Technical Papers. IEEE International, Feb 2008, pp. 150-603.

[27] Y. Kim, H. Kim, and H.-J. Yoo, "Electrical characterization of screen-printed circuits on the fabric," Advanced Packaging, IEEE Transactions on, vol. 33, no. 1, pp. 196-205, Feb 2010.

[28] J. Yoo and H.-J. Yoo, "Fabric circuit board-based dry electrode and its characteristics for long-term physiological signal recording," in Engineering in Medicine and Biology Society, EMBC, 2011 Annual International Conference of the IEEE, Aug 2011, pp. 2497-2500.

[29] J. Yoo, L. Yan, S. Lee, Y. Kim, and H.-J. Yoo, “A 5.2 mw self-configured wearable body sensor network controller and a $12 \mathrm{mu}$ w wirelessly powered sensor for a continuous health monitoring system," Solid-State Circuits, IEEE Journal of, vol. 45, no. 1, pp. 178-188, Jan 2010.

[30] J. Yoo, L. Yan, S. Lee, H. Kim, B. Kim, and H.-J. Yoo, "An attachable ecg sensor bandage with planar-fashionable circuit board," in Wearable Computers, 2009. ISWC '09. International Symposium on, Sept 2009, pp. 145-146.

[31] J. Yoo, L. Yan, S. Lee, H. Kim, and H.-J. Yoo, "A wearable ecg acquisition system with compact planar-fashionable circuit board-based shirt," Information Technology in Biomedicine, IEEE Transactions on, vol. 13, no. 6, pp. 897-902, Nov 2009.

[32] S. Lee, B. Kim, T. Roh, S. Hong, and H.-J. Yoo, "Arm-band type textile-mp3 player with multi-layer planar fashionable circuit board (p-fcb) techniques," in Wearable Computers (ISWC), 2010 International Symposium on, Oct 2010, pp. $1-7$. 
[33] L. Yan, J. Bae, S. Lee, T. Roh, K. Song, and H.-J. Yoo, "A 3.9 mw 25-electrode reconfigured sensor for wearable cardiac monitoring system," Solid-State Circuits, IEEE Journal of, vol. 46, no. 1, pp. 353-364, Jan 2011.

[34] H. Kim, Y. Kim, B. Kim, and H.-J. Yoo, "A wearable fabric computer by planarfashionable circuit board technique," in Wearable and Implantable Body Sensor Networks, 2009. BSN 2009. Sixth International Workshop on, June 2009, pp. 282-285.

[35] T.-H. Kang, C. Merritt, E. Grant, B. Pourdeyhimi, and H. Nagle, "Nonwoven fabric active electrodes for biopotential measurement during normal daily activity," Biomedical Engineering, IEEE Transactions on, vol. 55, no. 1, pp. 188-195, Jan 2008.

[36] T.-H. Kang, C. Merritt, B. Karaguzel, J. Wilson, P. Franzon, B. Pourdeyhimi, E. Grant, and T. Nagle, "Sensors on textile substrates for home-based healthcare monitoring," in Distributed Diagnosis and Home Healthcare, 2006. D2H2. 1st Transdisciplinary Conference on, April 2006, pp. 5-7.

[37] B. Karaguzel, C. Merritt, T. Kang, J. M. Wilson, H. Nagle, E. Grant, and B. Pourdeyhimi, "Flexible, durable printed electrical circuits," The Journal of The Textile Institute, vol. 100, no. 1, pp. 1-9, 2009.

[38] Y. Design, "Gps snowboarding jacket by nick ellrich," March 2007, accessed: 2015-10-23. [Online]. Available: http://www.yankodesign.com/2007/03/27/ gps-snowboarding-jacket-by-nick-ellrich/

[39] S. James, "Smart textile/fabric market to reach usd 1.59 billion by 2020: Grand view research, inc." August 2015, accessed: 2015-10-25. [Online]. Available: http://www.prnewswire.com/news-releases/ smart-textilefabric-market-to-reach-159-billion-by-2020-grand-view-research-inc-521228641. html/

[40] B. Ryan, "Wearable biosensors for firefighters: Wasp," in BME 281 Presentation, Oct 2014 . 
[41] F. Hilde, "Final report summary - safe@sea(protective clothing for improved safety and performance in the fisheries)," August 2013, accessed: 2015-10-25. [Online]. Available: http://cordis.europa.eu/result/rcn/57672_en.html

[42] S. Sagem, "Land defense sagem soldier system," accessed: 2015-10-25. [Online]. Available: http://www.sagem.com/land-defense/infantry/soldier-system

[43] O. smart textile technology, "Esa measuring astronauts' muscle activity," accessed: 2015-10-25. [Online]. Available: http://www.ohmatex.dk/?portfolio= esa-3

[44] C. S. Agency, "Astroskin: Canada's smart shirt for space," March 2015, accessed: 2015-10-25. [Online]. Available: http://www.asc-csa.gc.ca/eng/ sciences/astroskin.asp

[45] OMSignal, "Om smart shirt how it works," accessed: 2015-10-25. [Online]. Available: http://www.omsignal.com/pages/how-it-works

[46] P. van Dongen, "Werable solar," 2013, accessed: 2015-10-25. [Online]. Available: http://paulinevandongen.nl/projects/wearable-solar/

[47] tshirtOS, "About tshirtos why ballantine's and tshirtos?" accessed: 2015-10-25. [Online]. Available: http://www.tshirtos.com/about/

[48] Technovelgy, "Navjacket guides skiers with built-in display, headphones," accessed: 2015-10-29. [Online]. Available: http://www.technovelgy.com/ct/ Science-Fiction-News.asp?NewsNum=1354

[49] B. D., "These high-tech shirts and pants can help protect kids with autism," February 2015, accessed: 2015-10-25. [Online]. Available: http: //www.huffingtonpost.com/2015/02/18/autism-gps-device_n_6705368.html

[50] M. Stoppa and A. Chiolerio, "Wearable electronics and smart textiles: A critical review," Sensors, vol. 14, no. 7, p. 11957, 2014. [Online]. Available: http://www.mdpi.com/1424-8220/14/7/11957 
[51] M. Jiri, "The use of conductive threads in the production of experimental textile antennas," in Elektrotechnika, Informacne technologie, Aug 2009.

[52] E. Post, M. Orth, P. Russo, and N. Gershenfeld, "E-broidery: Design and fabrication of textile-based computing," IBM Systems Journal, vol. 39, no. 3.4, pp. 840-860, 2000.

[53] A. Rida, L. Yang, R. Vyas, and M. Tentzeris, "Conductive inkjet-printed antennas on flexible low-cost paper-based substrates for rfid and wsn applications," Antennas and Propagation Magazine, IEEE, vol. 51, no. 3, pp. 13-23, June 2009.

[54] M. Tentzeris, "Novel paper-based inkjet-printed antennas and wireless sensor modules," in Microwaves, Communications, Antennas and Electronic Systems, 2008. COMCAS 2008. IEEE International Conference on, May 2008, pp. 1-8.

[55] P. Holmes and R. Loasby, Handbook of Thick Film Technology. UK: Electrochemical, 1976.

[56] P. Tees, "Printum tees, sreen printing and embroidery," accessed: 2015-10-28. [Online]. Available: http://www.printnumtees.com/

[57] B. Stern, "Flora gps jacket," accessed: 2015-10-29. [Online]. Available: https://learn.adafruit.com/flora-gps-jacket

[58] Mando, "Gps basics," accessed: 2015-10-29. [Online]. Available: https: //learn.sparkfun.com/tutorials/gps-basics

[59] L. Boris, "Small breakout for sim900 gsm module," May 2011, accessed: 2015-10-29. [Online]. Available: http://www.open-electronics.org/ small-breakout-for-sim900-gsm-module/

[60] F. Zahradnik, "Wifi positioning system," accessed: 2015-10-29. [Online]. Available: http://gps.about.com/od/glossary/g/wifi_position.htm

[61] A. Rida, G. Shaker, F. Nasri, T. Reynolds, S. Nikolaou, and M. Tenzeris, "Inkjet printing of dual band conformal antenna for use in wifi frequency bands," in Radio and Wireless Symposium (RWS), 2010 IEEE, Jan 2010, pp. 65-67. 
[62] N. Salman, N. Alsindi, L. Mihaylova, and A. Kemp, "Super resolution wifi indoor localization and tracking," in Sensor Data Fusion: Trends, Solutions, Applications (SDF), 2014, Oct 2014, pp. 1-5.

[63] N. Hernandez, M. Ocana, J. Alonso, and E. Kim, "Wifi-based indoor localization and tracking of a moving device," in Ubiquitous Positioning Indoor Navigation and Location Based Service (UPINLBS), 2014, Nov 2014, pp. 281-289.

[64] M. Hoang, J. Schmalenstroeer, C. Drueke, D. Tran Vu, and R. Haeb-Umbach, "A hidden markov model for indoor user tracking based on wifi fingerprinting and step detection," in Signal Processing Conference (EUSIPCO), 2013 Proceedings of the 21st European, Sept 2013, pp. 1-5.

[65] S. Eisa, J. Peixoto, F. Meneses, and A. Moreira, "Removing useless aps and fingerprints from wifi indoor positioning radio maps," in Indoor Positioning and Indoor Navigation (IPIN), 2013 International Conference on, Oct 2013, pp. 1-7.

[66] A. Audun, "2.4ghz inverted f antenna," 2008.

[67] U. S. N. Institute, Naval Institute Proceedings. United States Naval Institute, 1910, no. v. 36, pt. 2. [Online]. Available: https://books.google.com.sa/books? id=KSNKAAAAYAAJ 


\section{APPENDICES}

\section{A Localization Device's Firmware and Software}

Microcontroller Atmega328P firmware.

\#include < Arduino.h $>$

\#include < SoftwareSerial.h $>$

\#include <avr/wdt.h>

\#define SSID "Localization"

\#define KEY "L0calSystem"

SoftwareSerial wiflyUart $(9,10)$;

String macs;

int ledPin $=7$;

int WDcount;

int respreceive(uint 8 _t $*$ buf, int timeout)

$\{\quad$ int read_bytes $=0$;

int ret;

unsigned long end_millis ; 


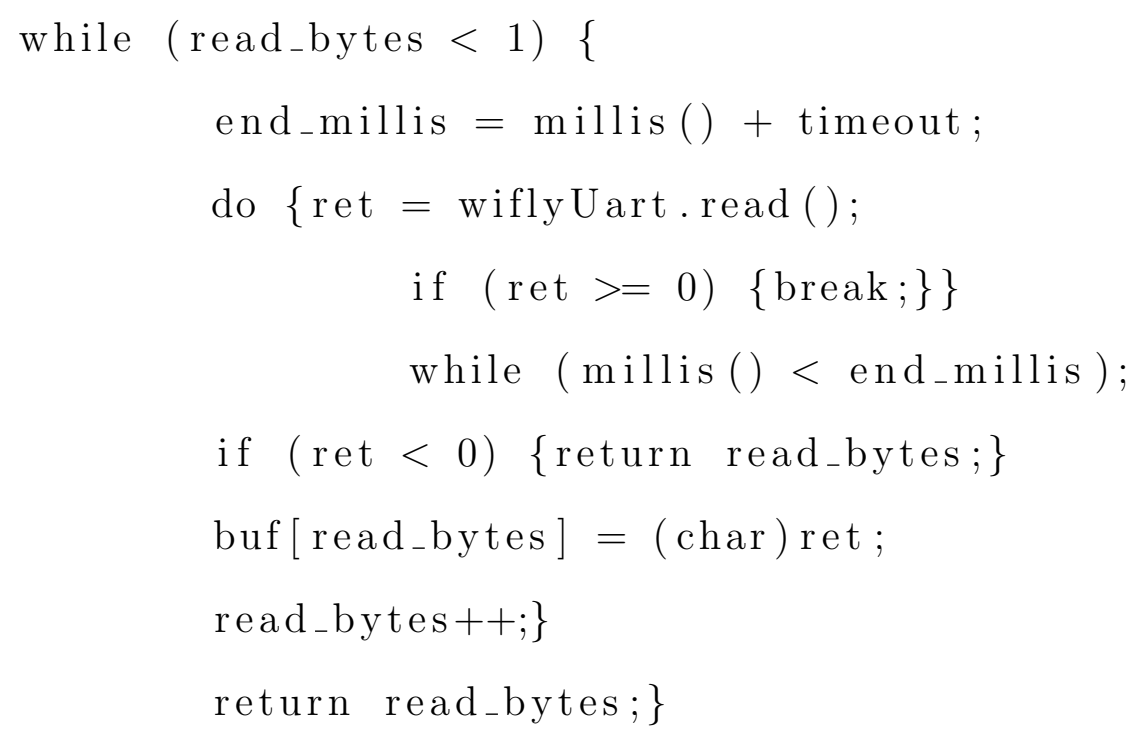

void ok_enforcer (String cmd)

$\{\quad$ String okstring $=" "$;

wdt_enable (WDTO_8S );

while (okstring.indexOf ("AOK")<0)

$\{\quad$ okstring $=$ cmd_manager $(\mathrm{cmd})$;

$\operatorname{delay}(100) ;\}$

wdt_disable ( ) ; 


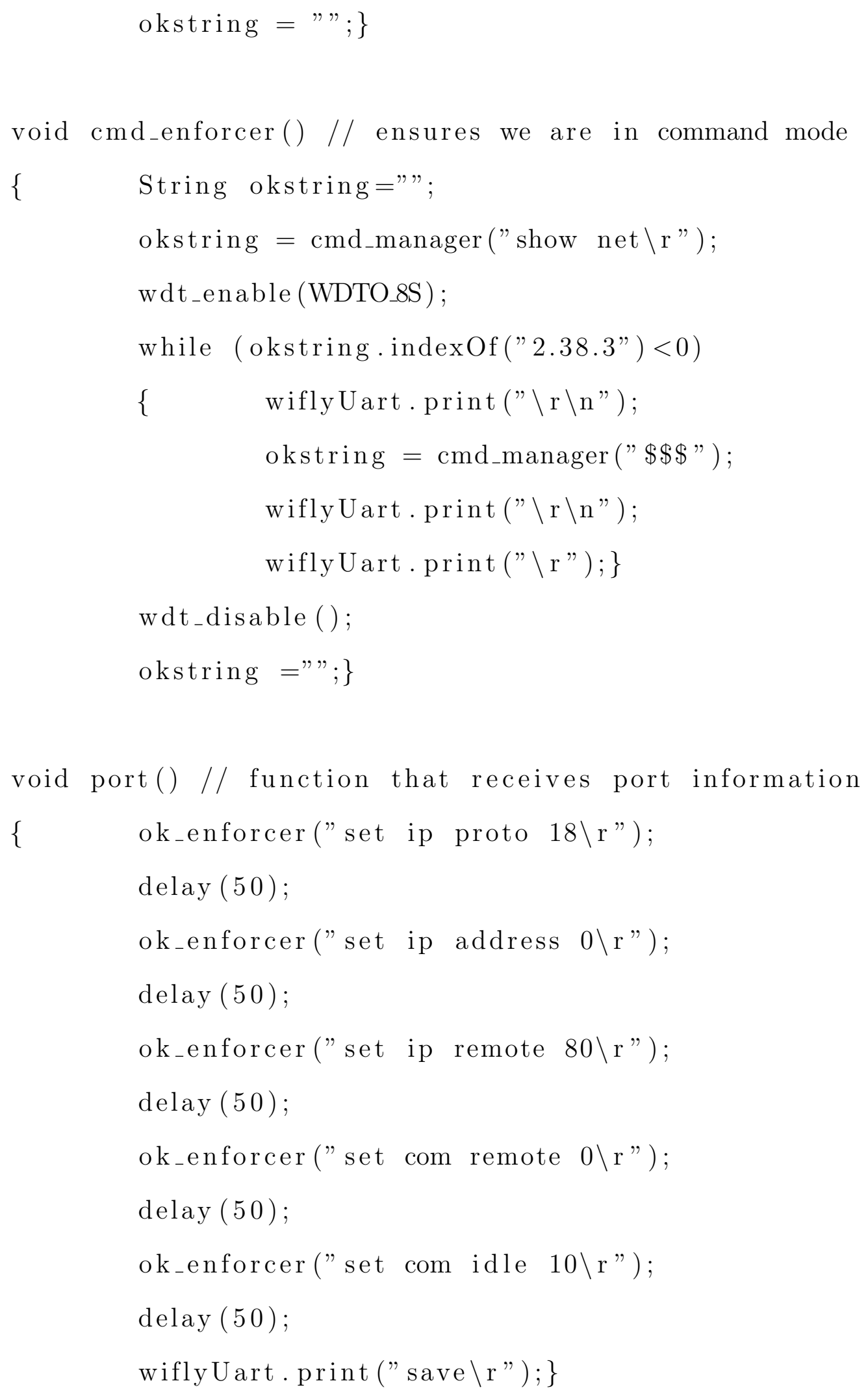


void doscan ()

\{ digitalWrite(ledPin, HIGH);

char c;

in $\operatorname{var}=0$;

int count $=0$;

String $\mathrm{ch}=" ”$;

String rssi="”;

String $\operatorname{resp}=" ” ;$

resp.reserve (700);

ch.reserve (5);

rssi.reserve (5);

wiflyUart.flush ( );

wifly Uart . print ("scan \r");

while (respreceive $\left(\left(\right.\right.$ uint $\left.\left.8 \_t *\right) \& c, 4000\right)>0$

$\& \& \operatorname{var} !=13)$

$\{\quad \operatorname{resp}+=\mathrm{c}$;

if $\quad\left(c==’ \backslash n^{\prime}\right)\{$ $\operatorname{var}++;\}\}$

$\operatorname{var}=0 ;$

for $\left(\right.$ int $\left.i=0 ; \quad \operatorname{resp}[i] !={ }^{\prime}, ' ; i++\right)\{$

$\operatorname{var}++;\}$

resp . remove $(0$, var -2$)$;

$\operatorname{var}=0$;

for $($ int $\quad \mathrm{i}=0 ; \quad \mathrm{i}<$ resp. length ()$; \quad \mathrm{i}++)\{$

if $\left(\operatorname{resp}[i]=={ }^{\prime} \backslash n^{\prime}\right)$

$\operatorname{var}++$;

if $(\operatorname{var}==10)\{$ 


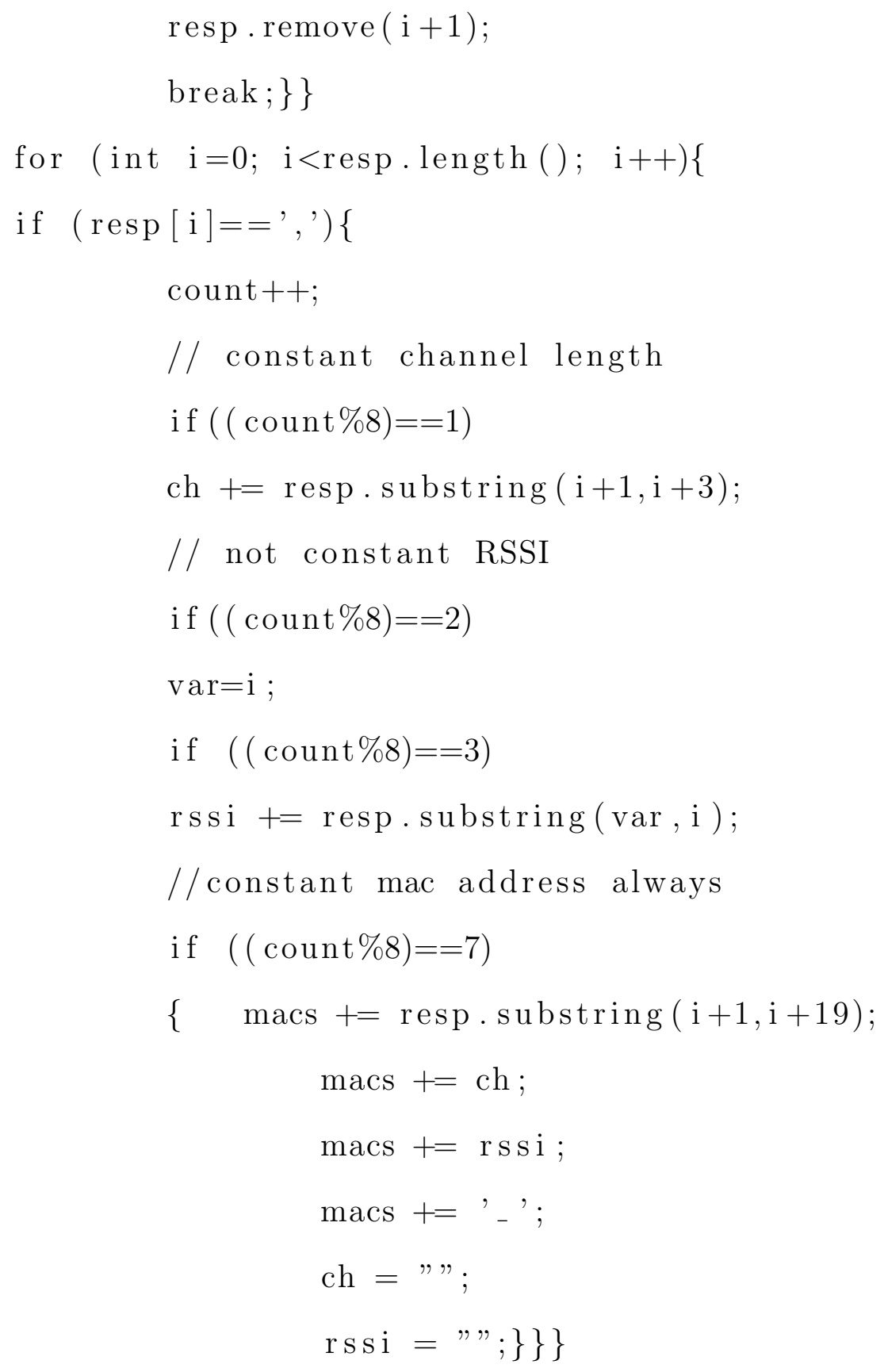


wifly Uart . print (macs);

Serial . print (macs);

macs $="$ GET / Test/wtrack $\cdot$ php? ID=smart\&W=";

wiflyUart.flush (); //important needed to escape v1 wiflyUart.print (" $\$ \$ \$) ; / /$ important needed to escape v1 digitalWrite (ledPin, LOW);

void wifi()// checks and joins the wifi

$\{\quad$ wiflyUart.flush ()$; / /$ flushing buffer

String okstring $="$;

okstring $=$ cmd_manager ("show net $\backslash \mathrm{r} ")$;

delay $(50)$;

// connectincting to wifi if not connected

if $($ okstring. indexOf $($ SSID $)<0)$

$\{\quad$ wiflyUart.print ("set w p ");

wifly Uart . print (KEY);

wifly Uart . print ("\r");

wiflyUart.print (" join ");

wifly Uart . print ( SSID);

wifly Uart . print ("\r");

delay $(500)$;

cmd_enforcer ( ); \}

okstring $="$;

okstring $=$ cmd_manager ("show net $\backslash \mathrm{r} "$ ");

while (okstring.indexOf("Auth=Fail")>0) \{

wiflyUart.print (" set w p");

wifly Uart . print (KEY); 


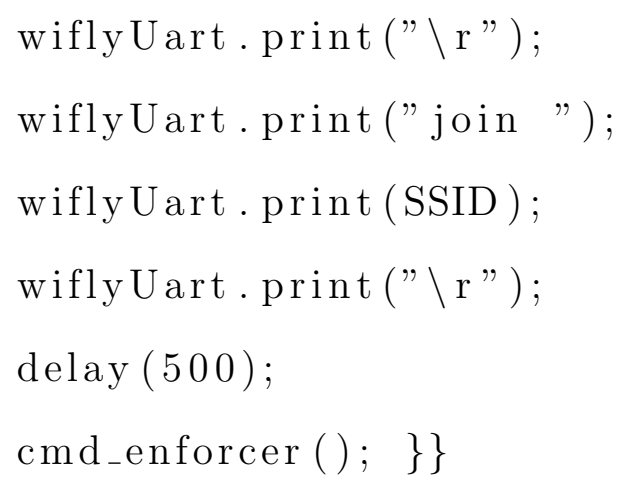

void authorization( $)\{/ /$ checking is association ok wiflyUart.flush();// flushing buffer String okstring ="”; 


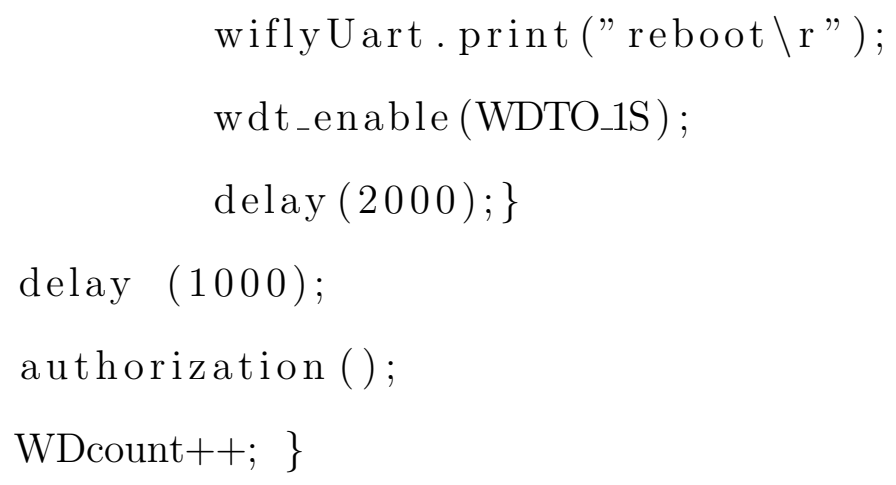




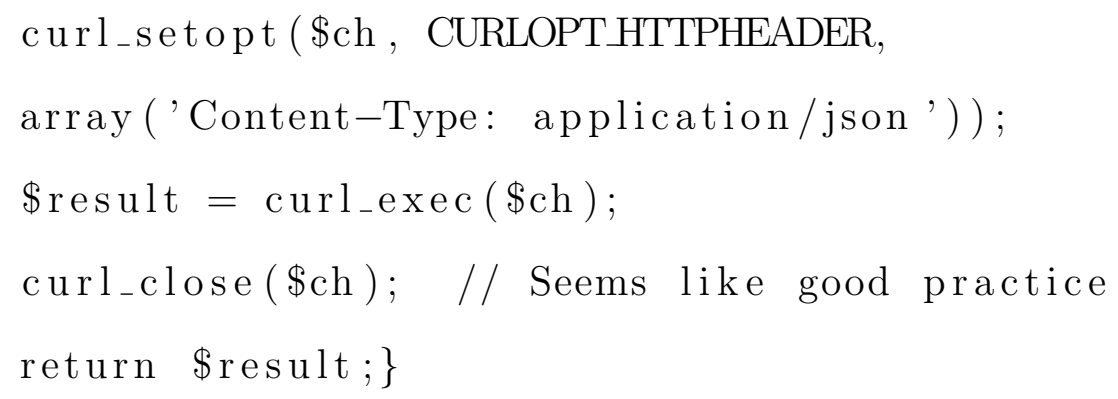




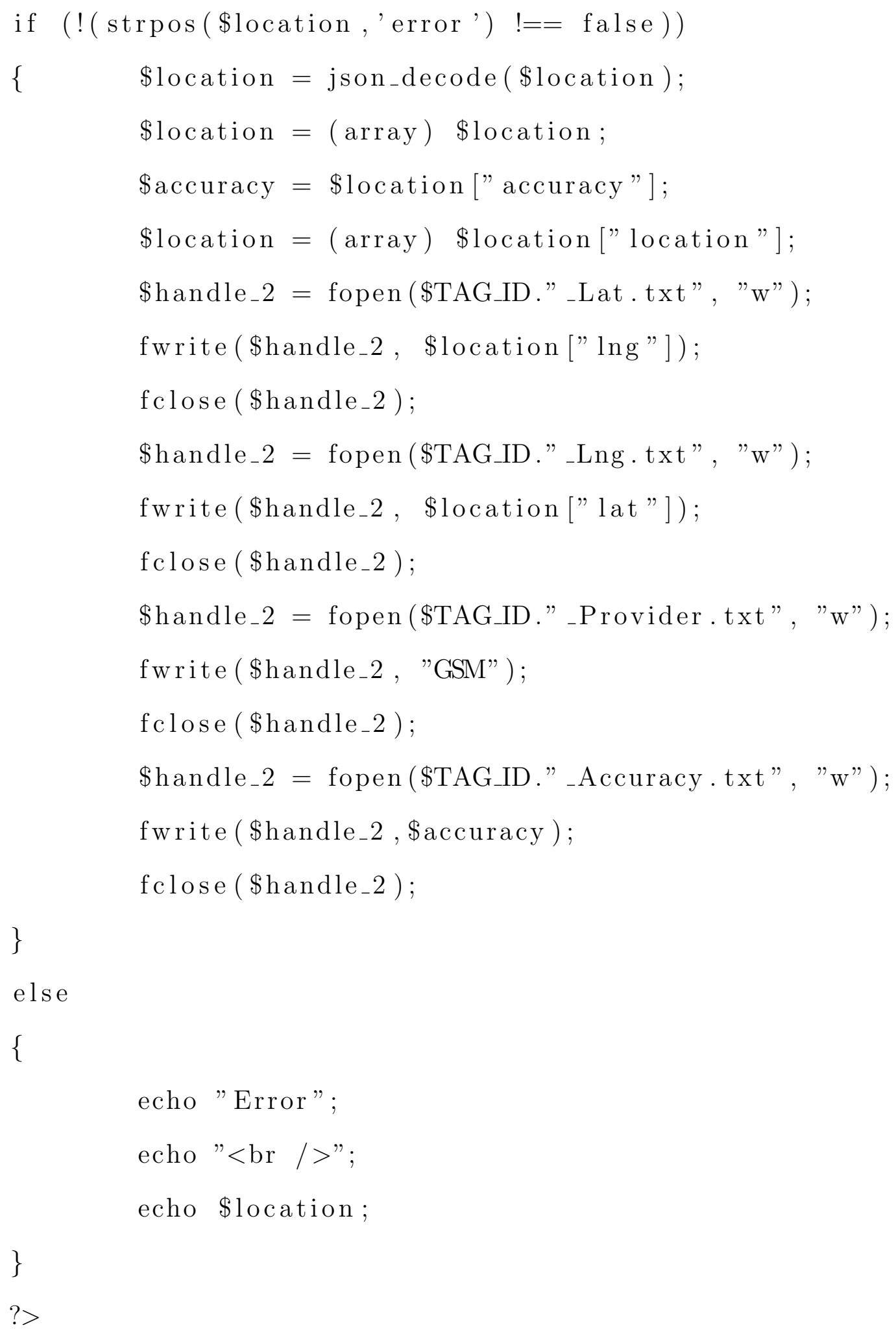

